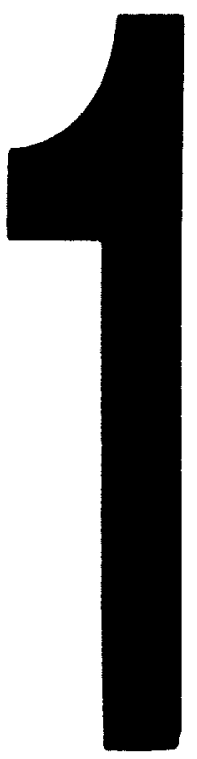

PM-1 3K" "x4" PHOTOGRAPHC MICROCOPY TARGET MaS 10101 AMBH/BO H2 EOUMALENT

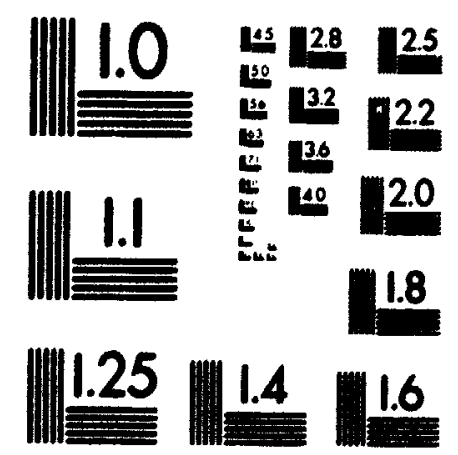

PRECLSON"m RESOUTION TARCETS

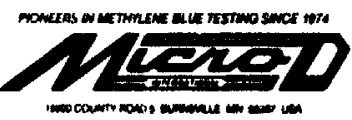

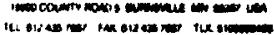


National Library

of Canada

Canadian Theses Service

Otowa. Canada

KIA ONA
Bibliothèque nationale

du Canada

Service des theses canadiennes
NOTICE

The quality of this microform is heavily dependent upon the quality of the original thesis submitted for microfilming. Every effort has been made to ensure the highest quality of reproduction possible.

If pages are missing, contact the university which granted the degree.

Some pages may have indistinct print especially it the original pages were typed with a poor typewriter ribbon or $H$ the university sent us an inferior photocopy.

Reproduction in full or in part of this microform is governed by the Canadian Copyright ACt, R.S.C. 1970, C. C-30, and subsequent amendments.

\begin{abstract}
AVIS
La qualité de cette microforme dépend grandement de la qualité de la thése soumise au microfilmage. Nous avons tout lait pour assurer une qualiné supérieure de reproduc. tion.

S'il manque des pages, veuillez communiquer avec runiversité qui a contéré le grade.

La qualité dimpression de certaines pages peut laisser à désirer, suntoui si les pages originales ont elé dactylogra. phiées a raide doun nuban usé ous si runiversité nous a fait parvenir une pholocopie de qualité in!érieure.
\end{abstract}

La reproduction, méme partielle, de cefte microlorme est soumise a la Loi canadienire sur le droit daauteur, SRC 1970, c. C-30, et ses amendements subséquents. 


\title{
ANALYSIS OF MONOLITHIC,
}

\section{FRACTIONAL-N FREQUENCY SYNTHESIZERS}

\author{
by
}

\author{
John Madsen
}

\author{
This thesis is submitted to the Faculty of Graduate Studies \\ and Research in partial fulfillment of the requirements \\ for the degree of
}

Master of Engineering

OCIEE

Department of Electronics

Faculty of Engineering

Carleton University

Ottawa, Canada

March, 1992

(C) copyright 1992, John Madsen 
National Library

of Canada

Canadian Theses Service

Bibliotheque nationale

du Canada

Service des thises canadiennes

Oanara Consoda

KiA ONA

The author has granted an irrevocable non. exclusive licence ellowing the National Lubrery of Cenada to reproduce, loen, distribute or sell copies of his/her thesis by any meens and in any form or format, making this thesis avaluble to interested persons.

The author retains ownership of the copyright in his/her thesis. Neither the thesis nor substantial extracts from it may be printed or otherwise reproduced without his/her permission.
L'auteur a accordé une licence krérocable et non exclusive permettent à la Blbiriotheque nationale du Canada de reprodure, prelor. distribuer ou vendie des coples de sa thise de quelque manibre et sous quelque forme que co soit pour mettre des exemplaires de cette thèse à la disposition des personnes intéressées.

L'auteur conserve la propriété du droit d'auteur qui protége sa these. Ni la these ni des extraits substantiels de celle-ci ne doivent Atre imprimés ou autrement reproduits sans son autorisation. 
The undersigned recommend to the Faculty of Graduate

Studies and Research the acceptance of the thesis:

\title{
ANALYSIS OF MONOLITHIC, FRACTIONAL-N FREQUENCY SYNTHESIZERS
}

\author{
submitted by John Madsen in partial fulfillment of the \\ requirements for the degree of Master of Engineering.
}

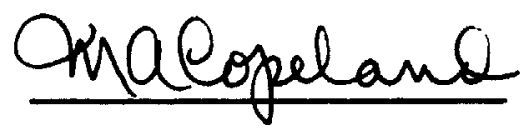

Prof. M.A. Copeland

Thesis Supervisor

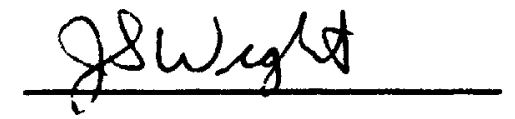

Prof. J.S. Wight

Chairman,

Department of Electronics

Carleton University

March, 1992 


\begin{abstract}
This thesis presents an investigation of the behaviour of a monolithic frequency synthesizer employing a sigma-delta modulator-based, fractional- $\mathrm{N}$ division technique. This technique has been shown t.. provide a region of low phase noise around the average frequency of the synthesizer output. The fundamental issue here is the generation of additional phase noise within this low-noise region due to the monolithic nature of the loop.

The main focus of this discussion is a charge-pump phase detector. It is shown that the sampled-data nature of this type of phase detector and the non-linear transfer function exhibited by typical monolithic versions are capable of generating additional phase noise near the average output frequency. These conclusions are validated by the results of a custom simulation model describing the time-domain behaviour of a fractional- $\mathrm{N}$ frequency synthesizer. This model can be used to determine if the increased phase noise is of concern for practical synthesizers once a high-quality, monolithic implementation of the system has been charactei ized.
\end{abstract}




\section{Acknowledgement}

The author would like to gratefully acknowledge the patient supervision and guidance of Prof. M.A. Copeland and the assistance of Tom Riley. 


\section{Contents}

List of Figures $\quad$ iv

List of Symbols viii

1 Introduction 1

1.1 Overview.......................... 1

1.2 Principles of Frequency Synthesis . . . . . . . . . . . . . 11

1.2.1 Basic Equations ...................... 11

1.3 Fractional-N Frequency Synthesis . . . . . . . . . . . . . 18

1.3.1 Digital-Phase Accumulator . . . . . . . . . . . . 23

1.3.2 Sigma-Delta Modulator ................. 26

1.4 Thesis Outline ........................ 27

2 Modelling a Monolithic Frequency Synthesizer 30 
2.1 Introduction $\ldots \ldots \ldots \ldots \ldots \ldots \ldots \ldots \ldots \ldots \ldots$

2.2 Monolithic Phase Detectors . . . . . . . . . . . 33

2.2.1 Charge-Pump Phace Detector . . . . . . . . . . 34

2.2.2 Sampling Phase Detectors . . . . . . . . . . . . . 39

2.2 .3 Comparison $\ldots \ldots \ldots \ldots \ldots \ldots \ldots \ldots \ldots \ldots \ldots \ldots$

2.3 Closed-loop Analysis . . . . . . . . . . . . . . . 45

2.3.1 Continuous-Time Equivalent . . . . . . . . . . . 47

2.3.2 Time-Domain Representation . . . . . . . . . . . 47

2.3.3 Frequency-Domain Representation $\ldots \ldots \ldots \ldots \ldots \ldots .33$

2.4 Summary $\ldots \ldots \ldots \ldots \ldots \ldots \ldots \ldots \ldots \ldots$

3 Sampled-Data and Non-Linear Aspects 87

3.1 Introduction $\ldots \ldots \ldots \ldots \ldots \ldots \ldots \ldots \ldots \ldots$

3.2 Sampling and Spectral Replication $\ldots \ldots \ldots \ldots \ldots \ldots \ldots$

3.2.1 Sampling and Replication in a Typical Context . . . . . 63

3.2.2 Sampling by a Phase Detector . . . . . . . . . . . 66

3.3 Non-Linear Charge-Pump Phase Detector . . . . . . . . . . 74

3.3.1 Asymmetrical Propagation Delays . . . . . . . . . . 76

3.3.2 Asymmetrical Current Sources . . . . . . . . . . 80 
3.4 Summary ............................ 82

4 Simulation Results 83

4.1 Introduction .......................... 83

4.2 Basic Loop Behaviour ... . . . . . . . . . . . . 85

4.3 Sampled-Data Characteristics ................... 94

4.4 Non-Linear Phase Detector . . . . . . . . . . . . . . . . 97

4.5 Summary ......................... 106

$\begin{array}{lll}5 & \text { Conclusions } & 108\end{array}$

A Source Code 114 


\section{List of Figures}

1.1 Indirect Frequency Synthesis $\ldots \ldots \ldots \ldots \ldots \ldots \ldots$

1.2 Divider Noise $n_{d}(t)$ due to Fractional-N Synthesis $\ldots \ldots \ldots \ldots$

1.3 Char ge-Pump Phase Detector (a) Schematic (b) Waveforms $\ldots \ldots 7$

1.4 Definition of Locp Quantities $\ldots \ldots \ldots \ldots \ldots \ldots$

1.5 Loop Filter (a) Schematic (b) Bode Plot $\ldots \ldots \ldots \ldots \ldots$

1.6 Fractional-N Frequency Synthesizer $\ldots \ldots \ldots \ldots \ldots \ldots$

1.7 Dual-Modulus Divider under External Control . . . . . . . . 20

1.8 Phase Deviation due to Divider Noise . . . . . . . . . 22

1.9 Digital Phase Accumulator (a) Block Diagram (b) Z-Domain Form . 24

1.10 Oversampled Analog-to-Digital Converter . . . . . . . . 28

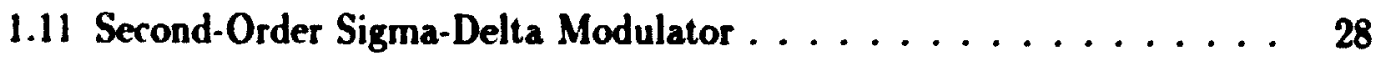

2.1 Charge-Pump Phase Detector (a) Schematic (b) Waveforms ... . 35 
2.2 Dual Outputs of PFD Combined into \& Single Signal . . . . . . . 37

2.3 Lead-Lag Filter with Additional Pole . . . . . . . . . . . . 37

2.4 Sample-and-Hold Phace Detector a) Schematic b) Output . . . . . . 41

2.5 Sinsoidal Phase Detector . . . . . . . . . . . . . . . . . . . . 42

2.6 Examples of Time-Domain Behaviour . . . . . . . . . . . 50

$2.7 V_{c}(t)$ for Negative $\Delta t[m-1]$ and $\Delta t[m] \ldots \ldots \ldots \ldots$

3.1 Ref ..., Div $\cdots$, and $\theta_{d}(t)---$ when $B=0.4$ and $\omega_{n}=0.2 * \omega_{i} \ldots 61$

3.2 Ref .... Div $\ldots$, and $\theta_{d}(t)---$ when $B=0.4$ and $\omega_{0}=1.1 * \omega_{i} \ldots \quad 63$

3.3 Sampling Stage (a) Schematic (b) Waveforms . . . . . . . . . . . 64

3.4 Basic Sample-and-Hold Circuit ... . . . . . . . . . . . . 66

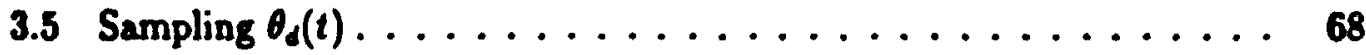

3.6 Ref ..., Div $\ldots$, and $\theta_{d}(t)-\ldots$ when $B=0.2$ and $\omega_{0}=3.0 * \omega_{i} \ldots 73$

3.7 Phase-Frequency Detector . . . . . . . . . . . . . . 78

3.8 Current into Loop Filter . . . . . . . . . . . . . . . . . . 78

$3.9 Q_{n}$ versus $\Delta t_{n}$ for Asymmetrical Propagation Delays . . . . . . 79

3.10 Modelling a Charge-Pump Phase Detector with Asymmetrical Propagation Delays .......................... 79

$3.11 Q_{n}$ versus $\Delta t_{n}$ for Asymmetrical Current Sources $\ldots \ldots \ldots 1$ 
4.1 Fractional-N Frequency Syntheaizer as Simulated (Frequencies are scaied by a factor of 10,000 from a typical application) . . . . . . . . 87

4.2 Second-Order SDM used in Simulations $(-10,000 \leq P \leq 10,000) \ldots 87$

4.3 wind $(t)$ when $P=1140$ (note: tolel duration is 0.3 rec) . . . . . . . 89

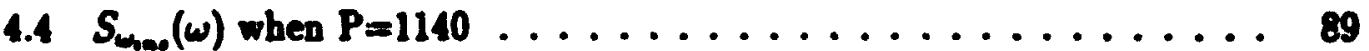

4.5 $S_{\text {wao }}(w)$ when P=1140 (Divider Controlled by a Digital Phase Accumulator) ............................. 90

4.6 Transient Loop Behaviour $-\omega_{\text {ino }}(t) \ldots \ldots$. . . . . . . . . . 92

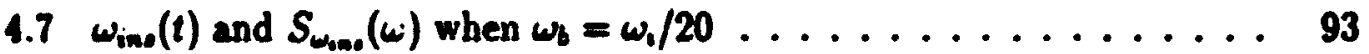

$4.8 S_{\omega_{100}}(\omega)$ when $\omega_{0 n}=0.3 * \omega_{i} \ldots \ldots \ldots . \ldots \ldots$

$4.9 S_{w_{10}}(\omega)$ when $w_{0_{n}}=1.1 * \omega_{i} \ldots \ldots \ldots . \ldots \ldots$

4.10 Non-Linear Phase Detector Transfer Function for Asymmetrical Propagation Delays....................... 98

4.11 Modelling the Propagation Delay Non-Linearity (a) Piecewise Linear Approximation (b) Time-Domain Behaviour . . . . . . . . . . 100

$4.12 \omega_{\text {inos }}(t)(a) t_{d s}=40 \mu s(b) t_{d}=0 \ldots \ldots \ldots . \ldots \ldots 1$

$4.13 S_{w_{100}}(w)(a) t_{d z}=40 \mu s(b) t_{d z}=0 \ldots \ldots \ldots 3$

4.14 Phase Detector Transfer Function with Current Source Non-Linearity 105 
$4.15 S_{\omega_{\text {ino }}}(\omega)$ - Phase Detector Includea Current Source Non-Linearity, $\omega_{0,}=$ $0.4 * w_{i} \ldots \ldots \ldots \ldots \ldots \ldots \ldots \ldots \ldots \ldots \ldots \ldots$

$4.16 S_{\text {waod }}(w)$ (a) with Current Source Non-Linearity (b) without Current

Source Non-Linearity . . . . . . . . . . . . . . . . . 107 


\section{List of Symbols}

$n_{d}(t) \ldots$ divider noise

$S_{d}(\omega) \ldots$ divider output noise spectrum

Ref ... reference signal

Div ... divider output

$\theta_{\text {Res }}(t) \ldots$ reference signal phase

$\theta_{D w}(t) \ldots$ divider output phase

$\theta_{d}(t) \ldots$ continuous phase difference between Ref and Div

$\theta_{d}^{*}(t)$. . phase detector output

$\omega_{o} . .$. VCO output frequency

$\omega_{1}$... reference frequency

$\omega_{c} \ldots$ V.OO centre frequency

$\kappa_{\circ} \ldots$ VCO gain

$v_{c}$... loop filter output voltage

$\omega_{f}$... divider output frequency

N ... divider ratio

$K_{o}^{\prime} \ldots$ effective VCO gain (includes divide ratio) 
$v_{p} \ldots$ phase detector output voltage

$K_{d} \ldots$ phase detector gain

$F(s)$.. loop filter transfer function

G(s) ...open-loop gain

$T(s)$... closed-loup gain

$\omega_{n} \ldots$ closed loop natural frequency

$\zeta$... damping factor

$\omega_{b}$... unity-gain bandwidth

$b(t) \ldots$ bit stream controlling dual-modulus divider

$\theta_{\Delta} \ldots$ peak phase deviation due to $n_{d}(t)$

$f_{c l k} \ldots$ clock frequency of digital phase accumulator

$\Delta t[m] \ldots$ time between $m^{\text {th }}$ edges of Ref and Div

T ... reference signal period

$t_{0} \ldots$ divider output period

$I_{p} \ldots$ charge-pump current source

$\omega_{\text {ino }}(t) \ldots$ instantaneous VCO output frequency

$s_{\omega_{\text {in }}}(\omega) \ldots$ frequency spectrum of $\omega_{\text {ins }}(t)$

$\omega_{\theta_{n}} \ldots$ frequency of sinusoidal phase noise

B ... magnitude of sinusoidal phase noise 
$\delta \ldots$ width of $n^{\text {th }}$ pulse of charge-pump phase detector output

Q ...charge transferred through the loop filter

$I_{p p} \ldots$ positive charge-pump current source

$I_{p m} \ldots$ negative charge-pump current source

P ... input to sigma-delta modulator 


\section{Chapter 1}

\section{Introduction}

\subsection{Overview}

This thesis presents an analytical investigation of the behaviour of a frequency synthesizer employing sigma-delta modulation, with particular emphasis on the aspects of a typical integrated circuit implementation. We will see that these aspects represent a potential source of error in the output which has not previously been identified. In order to assess the impact of this error, we have developed an accurate model of the time-domain behaviour of the circuit.

In the past, frequency synthesizers have been employed in a variety of communication systems based on applications requiring a stable, variable frequency source. The 
classical approach to indirect synthesis, shown in Figure 1.1 in block form, uses an integral divider in a feedback loop to generate output frequencies as multiples of the reference input. In order to achieve a reasonably small output channel spacing, the input frequency is typically thousands of times smaller than the frequencies being synthesized. Recently, as the demand for mobile communication services has increased, there has developed a need for these devices to be realized in integrated circuit form in order to minimize cost, area, and power consumption and this, in turn, has sparked interest in the use of a fractional- $\mathrm{N}$ approach.[1] If the integral divider of Figure 1.1 is replaced with one capable of rational division, a much larger reference frequency and a finer channel spacing are possible. This suggests that the loop bandwidth, essentially determined by the need to attenuate any spectral components at the reference frequency and its harmonics, can be increased accordingly and this larger value is the source of significant benefits for an integrated circuit implementation.

Specifically, a wider bandwidth implies faster settling of transients in response to changes in the divider count. Thus the additional circuitry often included to improve a loop's settling times would be unnecessary. Also, the input-referred phase noise of the VCO of Figure 1.1 undergoes a high-pass transfer function to the output and, as the loop bandwidth is increased, attenuation of this phase noise increases as well. With this in mind the requirements for a low-noise VCO could be relaxed in favour 


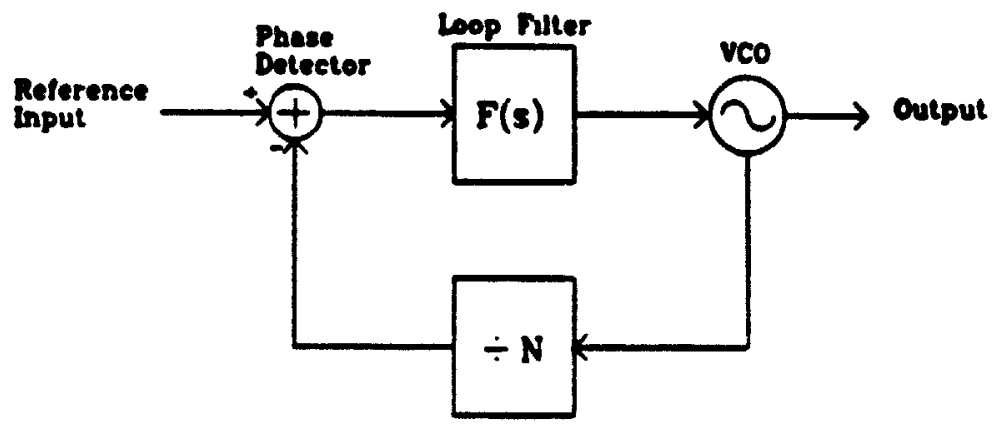

Figure 1.1: Indirect Frequency Synthesis

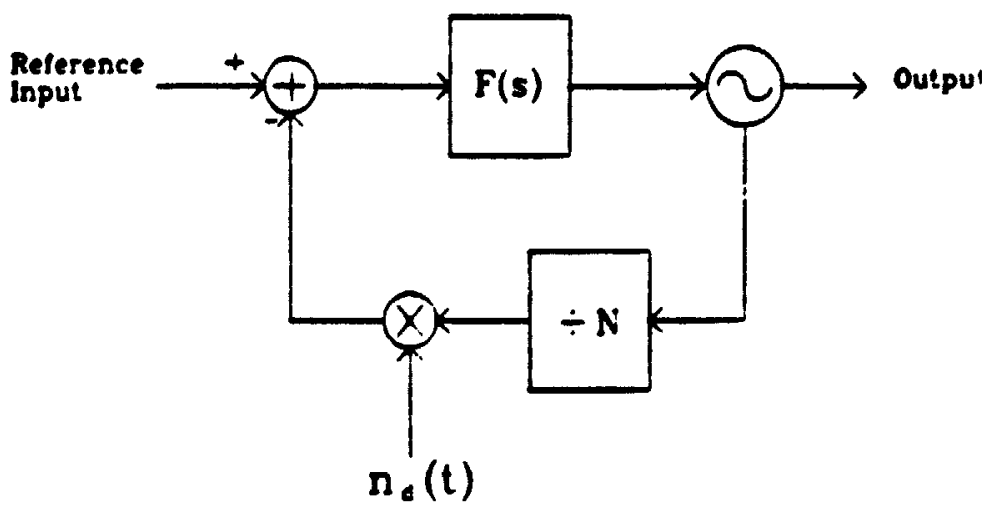

Figure 1.2: Divider Noise $n_{d}(t)$ due to Fractiond-A Syathesis 
of a VCO which is more easily integrated or is less complex. A third advantage is simply that any components at the reference frequency and its harmonics appearing at the output have been moved farther away from the desired frequency where they have less effect on the output.

In order to take advantage of the benefits of fractional- $\mathrm{N}$ synthesis, some means of realizing a rational divider is required. Several different techniques have been proposed, namely pulse swallowing, phase interpolation, and Wheatley Random Jittering. However, a new approach has been identified as the most suitable for a monolithic implementation, both in terms of the complexity of the circuitry involved and the phase noise content at the output.[2] This new approach is based on pulse swallowing, where a dual-modulus divider under some external control changes between two integer division ratios to produce, on average, the desired rational value. The fact that this value is only approached on a time-averaged basis suggests the presence of an unwanted time-dependent error. We can model this error, referred to in this thesis as divider noise $n_{d}(t)$, as a phase modulation of the average output of the divider output, as shown in Figure 1.2. Then the signal fed back to the phase detector is no longer a single frequency but consists of a dominant frequency, due to the average division ratio, and a two-sided noise spectrum $S_{d}(\omega)$. Dependir; on the loop bandwidth the noise spectrum may also appear in the spectrum of the VCO out- 
put. The new form of pulse swallowing uses a sigma-delta modulator as the divider controller to move the dominant frequency components of $S_{d}(\omega)$ away from the average value of the VCO output frequency. In uitively, we believe that this will improve the stability of the output frequency. This shaping of the noise spectrum has been confirmed by experimental results on a closed-loop version of discrete components.[1] The primary motivation for this thesis was io determine the nature of this noise and the shaping of its spectrum in the context of an integrated circuit implementation.

The specific context is determined by the use of a combination of a digital phase/frequency detector and a charge-pump as the phase detector of Figure 1.1. A simple schematic of this circuit, referred to in this thesis as a charge-pump phase detector, is given in Figure 1.3a. There are two aspects to the behaviour of this phase detector which are examined here. They are the introduction of sampled-data elements into the response of the loop and the effect of a non-linear trarsfer function of practical phase detectors of this type. Both are of interest because of our belief that they are capableof generating spurious signals at the output, leading to a degradation of the noiseshaping characteristics associated with sigma-delta modulation and of the stability of the output frequency. Consequently, the purpose of this thesis is to develop some insight into the relationship between a charge-pump phase detector and the frequency content of the VCO output, with special attention paid to the interaction between 
the phase detector and the divider noise.

In this discussion, the most important aspect of the behaviour of a charge-pump phase detector is the fact that the loop responds only to the differences in time between discrete events of the referenci an:d feedback signals, Ref and Div. Typically, Ref and Div are rectangular signals, as shown in Figure 1.3, and these discrete events are either the leading, or trailing, edges. An edge of either Ref or Div causes the charge-pump to be connected to the loop filter with charge transferred until the arrival of the corresponding edge of the other signal. The output of the phase detector consists of a train of current pulses, as shown in Figure 1.3b, and, because of this, the phase detector clearly does not provide a continuous measure of the phase difference between Ref and Div. To illustrate further, we will assume that, instead of rectangular signals, the nature of Ref and Div is such that we can define a continuous, time-varying phase, $\theta_{R e f}(t)$ and $\theta_{D i v}(t)$, for each. This makes it possible to define $\theta_{d}(t)$ as the continuous phase difference between Ref and Div, i.e. $\theta_{d}(t)=\theta_{R e f}(t)-\theta_{D i v}(t)$, and $\theta_{d}^{*}(t)$ as the output of the phase detector. In this case, the discrete events controlling the transfer of charge to the loop filter could be the zero-crossings of Ref and Div. If we assume that the differences in time between these events are small compared to the period of Ref, then the current pulses of $\theta_{d}^{*}(t)$ approach impulses and we can see a similarity to the ideal samples of a signal commonly referred to as sampled-data. 


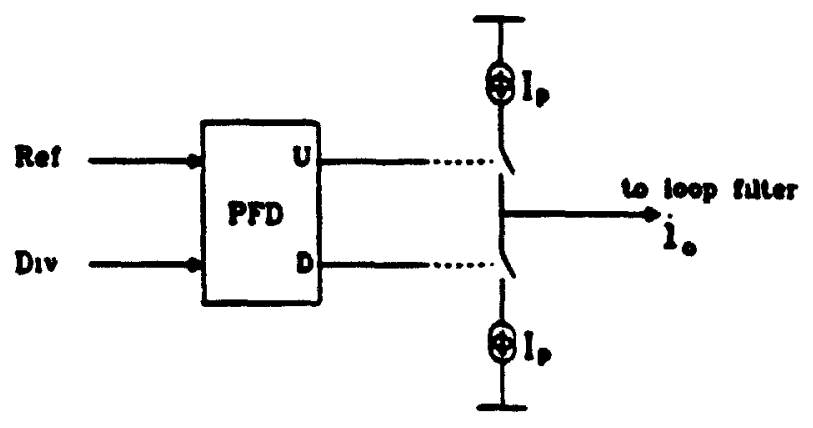

(a)

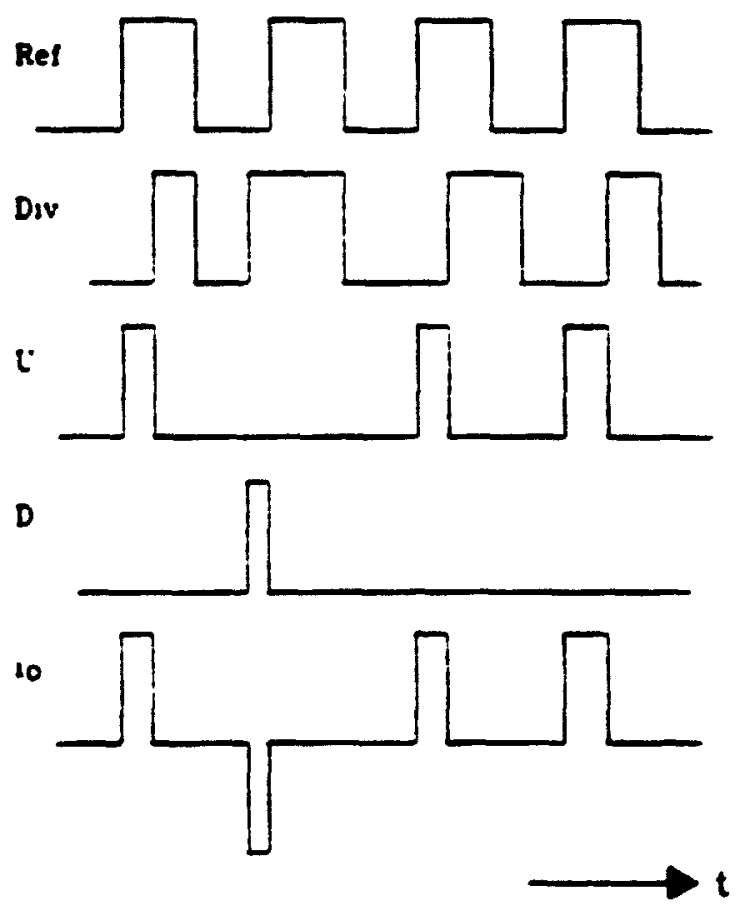

(b)

Figure 1.3: Charge-Pump Phase Detector (a) Schematic (b) Waveforms 
The fundamental difference between the samples of $\theta_{d}(t)$ and those of the output of a circuit like a switched-capacitor filter is that the value of the sample is conveyed in the width of the pulse rather than its height. Nevertheless, the loop must be considered to a certain degree as sampled-data and this fact suggests that one of the consequences of sampling, namely replications of the spectrum of the signal being sampled, is present. We know that, generally, the act of sampling generates replicas of the spectrum of the original signal around all multiples of the sampling frequency. In a frequency synthesizer context we are concerned that the spectrum of the phase detector output, $\theta_{d}^{*}(\omega)$, could contain replicas of $\theta_{d}(\omega)$ and that these rephicas might lead to an increase in phase noise in the VCO output over that of a continuous loop. In particular, components of $\theta_{d}(\omega)$ near multiples of the reference clock frequency will be replicated to low frequencies, possibly within the loop bandwidth and possibly within the region of noise-shaping around the output frequency.

Also of concern is the non-linear transfer function commonly exhibited by integrated charge-pump phase detectors. Typically, this non-linearity is attributed to non-ideal elements in the behaviour of the pinase/frequency detector or the chargepump and it is well-known that intermodulation of spectral components by a nonlinearity can lead to spurious components near dc. In a phase-locked loop, components near dc at the output of the phase detector translate to phase noise around 
the average output frequency. Consequently, we can see that the non-ideal behaviour of the phase detector can lead to increased phase noise and a degradation of the noise-shaping around the output frequency.

Furthermore, it seems conceivable that the sampled-data and non-linear aspects of the phase detector may interact; for the moment we will assume that the non-linearity of the phase detector can be represented as a third-order term in the polynomial

$$
Q=K_{1} \Delta t+K_{2}(\Delta t)^{3}
$$

where $Q$ is the amount of charge transferred to the loop filter by the charge-pump and $\Delta t$ is the time between edges of Ref and Div. The form of Equation 1.1 suggersts that $\theta_{d}(t)$ is sampled before the non-linearity occurs and that the non-linearity is due to the circuit used to convert this sample to a charge $Q$. In other words the non-linear components in $Q$ are not subject to replication by the sampling action of the phlase detector. However, Equation 1.1 can be rewritten as

$$
Q=K_{1}\left(\Delta t+K_{3} \Delta t^{3}\right)
$$

and this suggests that if $\Delta t$ is the actual time between edges of Ref and Div, the value of the sample has been distorted by an amount $K_{3} \Delta t^{3}$. In this case it seerns that the sampling of $\theta_{d}(t)$ follows the non-linearity and we would expect the result 
to be a further increase in phase noise at the output as the components due to the non-linearity are replicated throughout the spectrum.

Clearly, we need to develop a better understanding of the sampling implied by charge-pump phase detection and of its interaction with a non-linear transfer function. If we are to determine the effect of this type of phase detector on the output in other than a qualitative fashion we require some type of model of the circuit. Ideally we could derive a single, closed-form expression for the behaviour of the loop which could be transformed to the frequency domain. This is similar to the difference equation/z-transform approach used on typical sampled-data circuits. Unfortunately, we will see that, because of the nature of a charge-pump phase detector, such an expression can not be found, except possibly as a non-linear function of $\theta_{d}(t)$. Instead of attempting this approach, we have chosen to develop a simulation structure devoted to fractional- $\mathbf{N}$ frequency synthesizers employing charge-pump phase detectors. With this structure, the state of the loop is evaluated over a number of cycles of the reference input and a Discrete Fourier Transform is used to represent the time-domain information in the frequency domain.

A model of the type described above is generally of limited value unless it is accompanied by a more basic, intuitive understanding of why the circuit behaves as it does. Gardner has developed some of this intuitive background with a study of 
the effects of a charge-pump phase detector on the transient response and stability of a simple phase-locked loop.[6] In a frequency synthesizer application, we are more concerned with the steady-state frequency content at the output of the VCO and with the presence of spurious components which tend to corrupt the desired output frequency. As a result, in addition to the development of a time-domain model, we present a more theoretical discussion of the aspects of a monolithic implementation capable of generating these spurious components.

\subsection{Principles of Frequency Synthesis}

Much of the discussions of the following chapters, although meant to introduce aspects of charge-pump phzse detection and fractional- $\mathrm{N}$ division to frequency synthesis, are in terms of the parameters of a classical, continuous-time system. Thus a brief review is necessary.

\subsubsection{Basic Equations}

Analysis of a continuous PLL-based frequency synthesizer proceeds along the lines of a typical servo-control system and as is generally the case, the primary quantity of interest is the frequency-dependent, open-loop gain obtained by a Laplace transfor- 
mation of the time-domain behaviour.

Referring to Figure 1.4, the output frequency is given by

$$
\omega_{o}=\omega_{c}+K_{o} v_{c}
$$

where $\omega_{c}$ is the centre frequency of the VCO and $K_{0}$ is the VCO gain measured in $\mathrm{rad} / \mathrm{sec}-\mathrm{V}$. Then the frequency of the divider output is

$$
\omega_{f}=\frac{\omega_{c}+K_{o} v_{c}}{N}
$$

where $\mathrm{N}$ is the divider ratio in the feedback loop. If the changes in $\mathrm{N}$ are small compared to its central value Equation 1.4 can be rewritten as

$$
\omega_{f} \approx \omega_{i}+\frac{K_{o} v_{c}}{N}
$$

where $\omega_{1}$ is the reference frequency.

There are two important points concerning analysis of a phase-locked loop that should be noted here. First is the concept of the syste:n input being the phase of the reference signal rather than its amplitude. Thus the practical input and output signals do not explicitly represent the phases of interest and the phase detector must infer these quantities from some characteristic of the signals. Second, in absolute terms the phase of a signal with a constant frequency is a ramp. However, if all phases within the system are referred to a signal with a fixed frequency, then any 


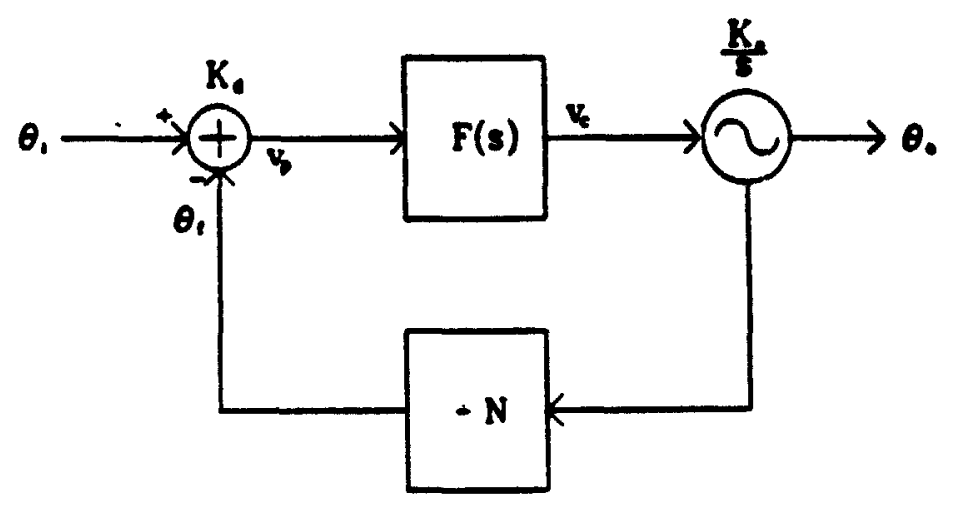

Figure 1.4: Definition of Loop Quantities

signal changing at that same frequency can only have a dc phase i.e. the signal is offset in time from the reference by a fixed amount. Similarly, a signal with a fixed frequency different than the reference will have a phase which is ramping in time and the slope of the ramp is given by the difference in frequencies.

Rewriting Equation 1.5 as

$$
\frac{d \theta_{f}}{d t} \approx \omega_{s}+\frac{K_{o} v_{c}}{N}
$$

we can see that further analysis is greatly simplified if the input frequency is chosen as the phase reference. Then equation 1.6 becomes

$$
\frac{d \theta_{f}}{d t}=\frac{K_{o} v_{c}}{N}
$$

For this simple discussion we will combine the VCO gain and division ratio to give 
an effective VCO gain of

$$
K_{o}^{\prime \prime}=\frac{K_{0}}{N}
$$

Then

$$
\frac{d \theta_{j}}{d t}=K_{o}^{\prime} v_{c}
$$

The output of the phase detector is

$$
v_{p}=K_{d}\left(\theta_{i}-\theta_{f}\right)
$$

where $K_{d}$ is the gain of the phase detector, assumed to be linear.

Transforming to the complex domain gives

$$
\begin{aligned}
V_{p}(s) & =K_{d}\left[\theta_{i}(s)-\theta_{f}(s)\right] \\
\theta_{f}(s) & =\frac{K_{0}^{\prime} V_{c}(s)}{s} \\
V_{c}(s) & =F(s) V_{p}(s)
\end{aligned}
$$

where $F(s)$ represents the transier function of the loop filter.

Equations $1.11,1.12$, and 1.12 can be combined to give the open-loop gain of the system as

$$
G(s)=\frac{K_{d} K_{o}^{\prime} F(s)}{s}
$$

and the closed-loop gain is

$$
T(s)=-\frac{K_{d} K_{o}^{\prime} F(s)}{s+K_{d}^{\prime} K_{o}^{\prime} F(s)}
$$


We can see that, at dc, the loop gain is infinite, even when no loop filter is present. As expected, this implies that the dc phase difference between the input and feedback signals is zero, i.e. the average frequencies of these signals are identical.

The loop is completely specified when the transfer function of the loop filter is determined; the filter in the synthesizer to be considered later is shown in Figure 1.5 along with a Bode plot of its transfer function. At low frequencies the filter acts as an integrator and has a zero at $\omega_{2}=1 / R_{1} C_{1}$ to ensure loop stability. The pole at $\omega_{3}=1 / R_{1} C_{2}$ increases attenuation of frequency components beyond the loop bandwidth. If $C_{2}$ is small compared to $C_{1}$ this attenuation is of consequence only at high frequencies. At frequencies comparable to the loop bandwidth the filter reduces to the active lead-lag configuration common to second-order loops and $F(s)$ is

$$
F(s)=-\frac{s \tau_{2}+1}{s \tau_{1}}
$$

Then the closed-loop transfer function of the loop becomes

$$
T(s)=\frac{K_{d} K_{o}^{\prime}\left(s \tau_{2}+1\right) / \tau_{1}}{s^{2}+s\left(K_{d} K_{o}^{\prime} \tau_{2} / \tau_{1}\right)+K_{d} K_{o}^{\prime} / \tau_{1}}
$$

Making use of servo-system terminology

$$
T(s)=\frac{2 \zeta \omega_{n} s+\omega_{n}^{2}}{s^{2}+2 \zeta \omega_{n} s+\omega_{n}^{2}}
$$




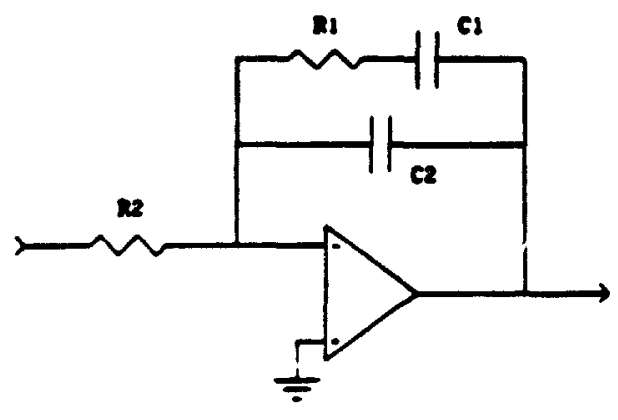

(a)

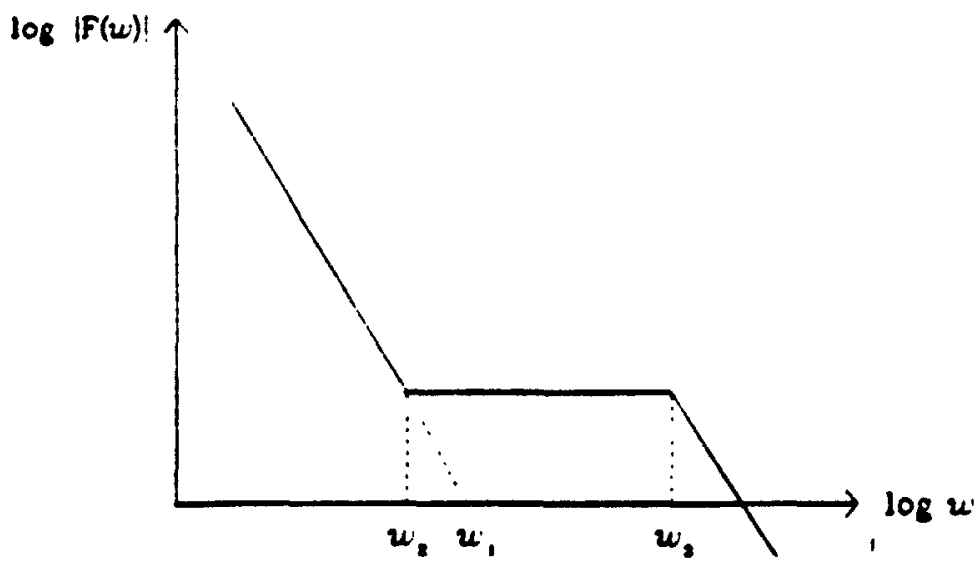

(b)

Figure 1.5: Loop Filter (a) Schematic (b) Bode Plot 
where the natural frequency, $\omega_{n}$, and the damping factor, $\zeta$, are given respectively by

$$
\begin{gathered}
\omega_{n}=\left(\frac{K_{d} K_{o}^{\prime}}{\tau_{1}}\right)^{1 / 2} \\
\zeta=\frac{\tau_{2} \omega_{n}}{2}
\end{gathered}
$$

When $\zeta \geq 0.5$ the open-loop unity-gain frequency and the natural frequency are related by

$$
\omega_{b}=2 \zeta \omega_{n}
$$

Equation 1.17 indicates that the reference input sees a low-pass transfer function to the output. In other words, the loop is basically unable to track the components of the phase of the reference signal above the loop bandwidth, $\omega_{b}$. From our point of view, what is more important is the fact that the phase difference between the reference and feedback signals sees the gain of the forward path as the transfer function to the output and this also has the form of a low-pass filter. As a result, generally we expect that components of $\theta_{d}(\omega)$ above the loop bandwidth would have little effect on the output. In a later chapter we will see that this is not necessarily true when the loop is implemented in monolithic form. 


\subsection{Fractional-N Frequency Synthesis}

This section continues the review of fundamental concepts with an examination of the pulse swallowing approach to fractional-N, indirect frequency synthesis. Figure 1.6 shows a $n / n+1$ divider, under control of an external bit stream, $b(t)$, in the feedback path of a phase-locked loop. The loop is capable of synthesizing the frequencies $f_{0}=f_{i} *(n+r)$ where $f_{i}$ is the input frequency and $\mathrm{r}$ is a fraction between 0 and 1. However, the output frequency takes on its desired value only on a time-averaged basis; at any particular time there is a phase error present in the loop since the division ratio is actually $n$ or $n+1$. We have chosen to refer to this error as divider noise and the intent of this section is to develop some insight into the relationship between the divider noise and the device controlling the divider.

For the sake of simplicity, we will base this discussion on the open-loop configuration of Figure 1.7, where a fixed frequency source is applied to the dual-modulus divider. If the frequency of the source is $f_{\bullet}$, the average frequency of the divider output is given by $f_{a v}=f_{s} /(n+r)$ where, again, $r$ is the desired fraction between 0 and 1. As an example, consider a $20 / 21$ divider and a required division ratio of 20.4 . The most obvious way to synthesize this ratio would seem to be to continuously repeat a pattern of division by 20 six times followed by division by 21 four times. For every 204 
cycles of the divider input, 10 cycles of the output would be completed. A spectrum analyzer on the output of the divider would show a strong component at the desired frequency of $f_{0} / 20.4$ but it would also reflect how this frequency is realized,i.e. by the divider output changing between two fixed frequencies, $f_{0} / 20$ and $f_{0} / 21$. Since the pattern of change between these frequencies is periodic, the spectrum will also contain other strong components and we are interested in determining the frequencies of these components relative to $f_{a v}$.

In order to do this, we find it helpful to model the effect of the fractional- $\mathrm{N}$ division as a modulation of the phase of the average output of the divider by the divider noise. Mathematically

$$
D i v=A * \operatorname{sgn}\left[\cos \left(\omega_{a v} t+\theta_{\Delta} * n_{d}(t)\right)\right]
$$

where $A$ is the amplitude of the divider output, $n_{d}(t)$ is the divider noise, and $\theta_{\Delta}$ is the peak phase deviation due to $n_{d}(t)$. Based on this definition, the divider noise is a normalized, dimensionless quantity between +1 and -1 . Then, it can be shown [3] that when the phase deviation, $\theta_{\Delta} * n_{d}(t)$, is small compared to $\omega_{a v} t$, the power spectrum of Div contains a component at $\omega_{a v}$ as well as the two-sided spectrum $N_{d}(\omega)$ centered on $\omega_{a v}$. As a result, if we are able to determine $N_{d}(\omega)$, we can make an evaluation of the effect of the divider noise on the output. 


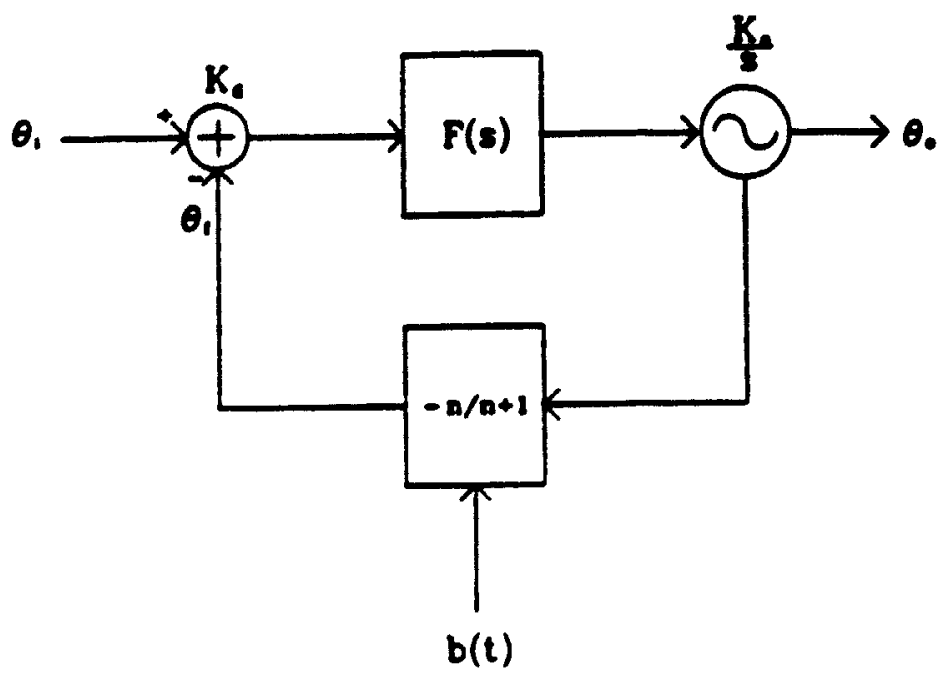

Figure 1.6: Fractional-N Frequency Synthesizer

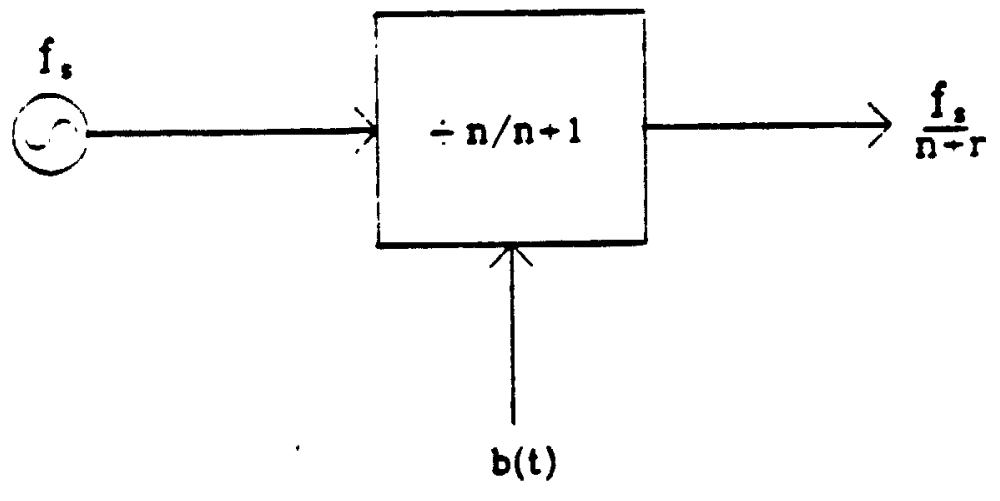

Figure 1.7: Dual-Modulus Divider under External Control 
In the discussion that follows we do not have to be concerned with the exact nat ure of $N_{d}(\omega)$; the location of the dominant components should be sufficient to make a qualitative judgment of the effect of $N_{d}(\omega)$. We can establish the fundamental nature of $N_{d}(\omega)$ by noting that, when the divide ratio is $\mathrm{n}$, we can express the phase deviation in the divider output as

$$
\theta_{\Delta} * n_{d}(t)=\omega_{a v} t-\frac{\omega_{p}}{n} t
$$

The equivalent of Equation 1.22 when the division ratio is $\mathrm{n}+1$ is

$$
\theta_{\Delta} * n_{d}(t)=\omega_{a v} t-\frac{\omega_{s}}{n+1} t
$$

Equations 1.22 and 1.23 are linear functions of time and are shown in Figure 1.8 corresponding to the example of division by 20.4. The important point is that, since the pattern of change of the divide ratio is periodic, the divider noise should exhibit the same periodicity. In other words, the pattern of divisions repeats every ten cycles of the divider output which implies that the fundamental of $N_{d}(\omega)$ is also $f_{a v} / 10$. Consequently, we expect the spectrum of the divider output to contain components at $f_{a v}$ and $f_{a v} \pm K * f_{a v} / 10$ where $K=2,4,6 \ldots$ and accounts for the triangular nature of the phase deviation.

The problem with using the simple technique described here to control the divider is that the spectrum of Div due to $N_{d}(\omega)$ can be heavily dependent on the desired 


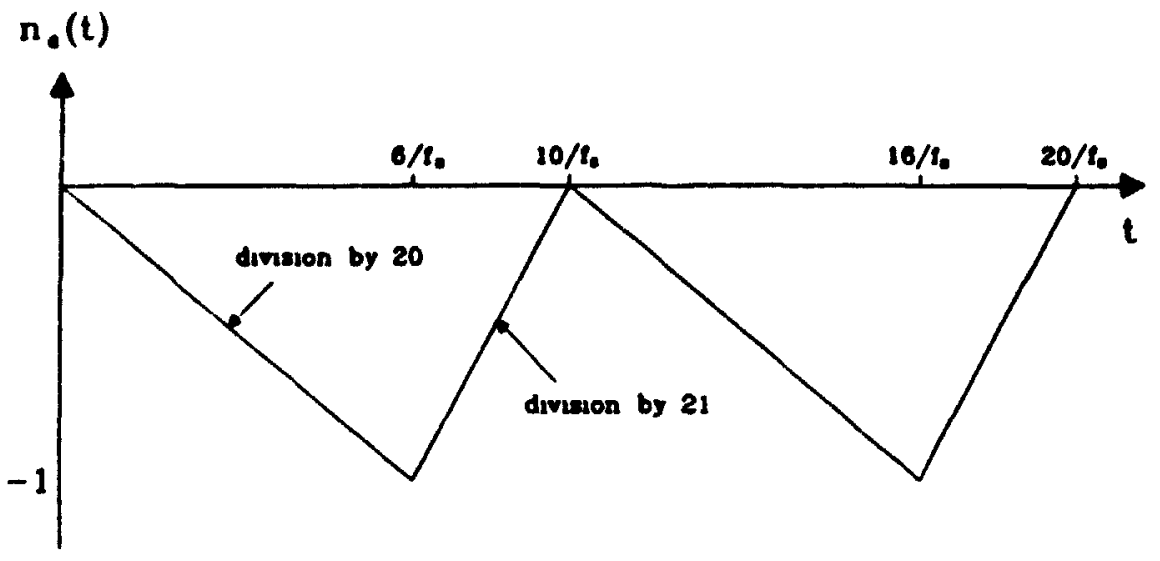

Figure 1.8: Phase Deviation due to Divider Noise

frequency. If we consider a division ratio of 20.39 rather than 20.4, a pattern of 61 divisions by 20 and 39 divisions by 21 is required and the fundamental of $N_{d}(\omega)$ is at a frequency ten times smaller than the previous case. If we extend this argument to division ratios requiring much longer patterns we can see that $N_{d}(\omega)$ may contain components at frequencies close to the desired output frequency. Intuitively, we believe that, as the main components of $N_{d}(\omega)$ move closer to the average output frequency, the stability of that frequency will decrease. The next sections describe two circuits, a digital phase accumulaior(DPA) and a sigma-delta modulator(SDM), suitable for use as the divider cuntroller by virtue of their ability to move the main components of $N_{d}(\omega)$ to higher frequencies where they will have less of an impact on 
the output.

\subsubsection{Dipital-Phase Accumulator}

The original approach to pulse swallowing made use of a digital phase accumulator as the divider controller.(Figure 1.9) Its output is a binary bit stream where a 'one' indicates division by the higher divider modulus and a 'zero' indicates division by the lower. The main element of the DPA is an m-bit digital accumulator. On every cycle of the clock signal a constant value, $K$, representing the desired fractional value, is added to the accumulator. When the accumulator overflows, the limit of its output range, $2^{m}$, is subtracted and a 'one' is produced at the output. Otherwise the output is a 'zero'. It can be shown that the average value of the bit stream is given by the ratio of the constant input to the limit of the output range, $\frac{K}{2^{m}} .[1]$

Once again, the characteristics of $N_{d}(\omega)$ and of the divider output spectrum can be demonstrated with an example. Assume that the average division ratio is to be 20.25 . This means that the value of the input, $K$, is one quarter of the output range of the accumulator. In other words, the accumulator will overflow on every fourth cycle of the DPA clock and a 'one' is generated. Since the content of the accumulator returns to zero on the fourth cycle, the pattern of three divisions by $\mathbf{2 0}$ and one division by 


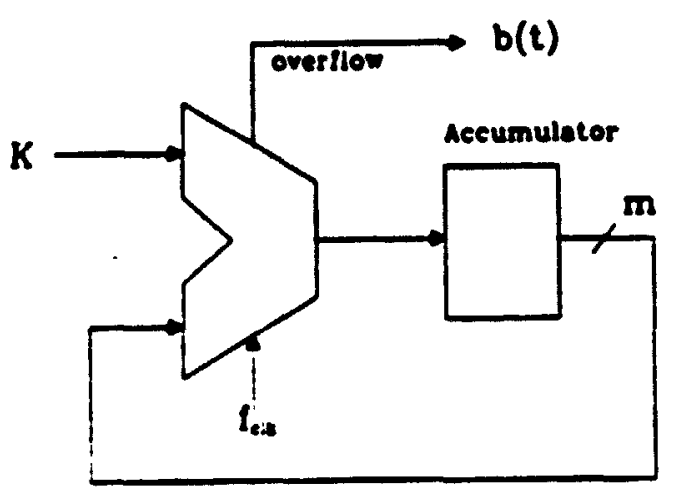

(a)

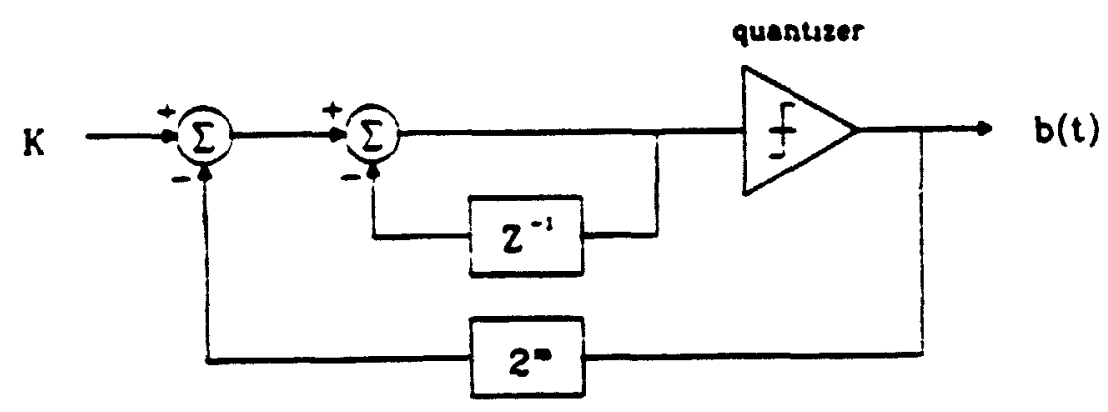

(b)

Figure 1.9: Digital Phase Accumulator (a) Block Diagram (b) Z-Domain Form 
21 is repeated continuously, i.e. the fundamental of $N_{d}(\omega)$ is at $\frac{L_{1}}{4}$ where $f_{c l k}$ is the frequency of the clock controlling the DPA. Then, besides a strong component at the desired frequency, $f_{a v}$, a spectrum analyzer would show other components at $f_{a v}+\frac{L_{a k}}{4}$ and $f_{a v}-\frac{L_{a k}}{4}$. However, when the DPA input is, say, one twentieth of $k$, corresponding to a division ratio of $20.05, N_{d}(\omega)$ contains a fundamental at a much lower frequency so, once again, the divider noise is heavily dependent on the required division ratio.

Despite this fact, the DPA does have an advantage over the simple approach to controlling the divider described earlier. Consider a division ratio of 20.525 . This can be realized with a pattern of 19 divisions by 20 followed by 21 divisions by 21 and the fundamental component of $N_{d}(\omega)$ is located at $\frac{t_{14}}{40}$. The DPA also produces a 40. bit pattern so the fundamental of $N_{d}(\omega)$ is located at the same frequency. However, during each 40-bit pattern, the accumulator will overflow 21 times. As a result the bit stream is more strongly periodic at higher frequencies with the result being that the dominant components of $N_{d}(\omega)$ are moved away from the average frequency of the output. This idea of 'shaping' $N_{d}(\omega)$ so that the divider noise has less of an effect on the output can be carried one step further with the use of a second-order sigma-delta modulator described below. 


\subsubsection{Sigma-Delta Modulator}

A continuous version second-order sigma-delta modulator is shown in Figure 1.10. Until recently the main area of application of these circuits has been as oversampled analog-to-digital converters. In this context the integrators in the forward path are continuous and a one-bit quantizer stage is used to convert the analog output of the second integrator to a bit stream. The average value of this bit stream corresponds to the analog input. As well, the large, low-frequency gain of the integrators in the forward path causes the main spectral components of the error associated with this conversion to occur at frequencies well-away from the average value of the output. However, if the analog integrators of Figure 1.10 are replaced with digital accumulators and the overflow from the second accumulator is used as the quantizer, as shown in Figure 1.11, we obtain a circuit very similar to the digital phase accumulator. In fact it has been shown that a DPA can be considered as a first-order sigma-delta modulator. [1] This suggests that a second-order modulator might be a good candidate for use as a divider controller. Experimental results show that this is true and that the performance of a sigma-delta modulator used as a divider controller is superior to that of a DPA. The presence of the second accumulator causes chere to be greater attenuation of the low-frequency components of $N_{d}(\omega)$; as well, the SDM 
introduces an element of randomness to the pattern of bits applied to the divider which reduces the dependence of $N_{d}(\omega)$ on the desired division ratio. Theory predicts a $40 \mathrm{db} / \mathrm{dec}$ shaping of the quantization noise for a second-order SDM. [2] The noise-shaping is further enhanced by the low-pass filtering action of the forward path of a closed loop. Consequently, the remainder of this thesis focuses on the sigma-delta modulator approach to fractional- $\mathrm{N}$ synthesis.

\subsection{Thesis Outline}

The ordering of topics presented in this thesis reflects the evolution of our thinking in one area of the broader study of monolithic, fractional- $\mathrm{N}$ frequency synthesizers. We began by recognizing the importance of these devices and by realizing that we required some means of analytically determining their behaviour. As well, we were aware that a monolithic implementation would commonly make use of a charge-pump, phase detector. Then, in order to properly represent the behaviour of the loop, we found that the standard procedures for closed-loop analysis were inappropriate and that a new approach was necessary. The resultant model of the loop is described in Chapter 2. However, during the development of this model, it also became apparent that the use of a charge-pump phase detector has implications for the fundamental 


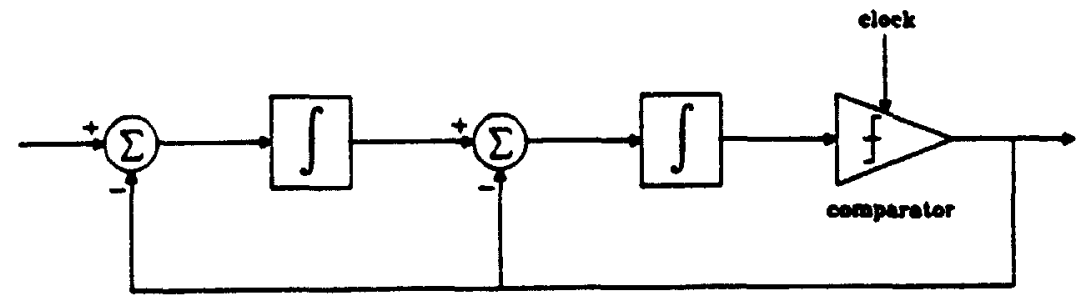

Figure 1.10: Oversampled Analog-to-Digital Converter

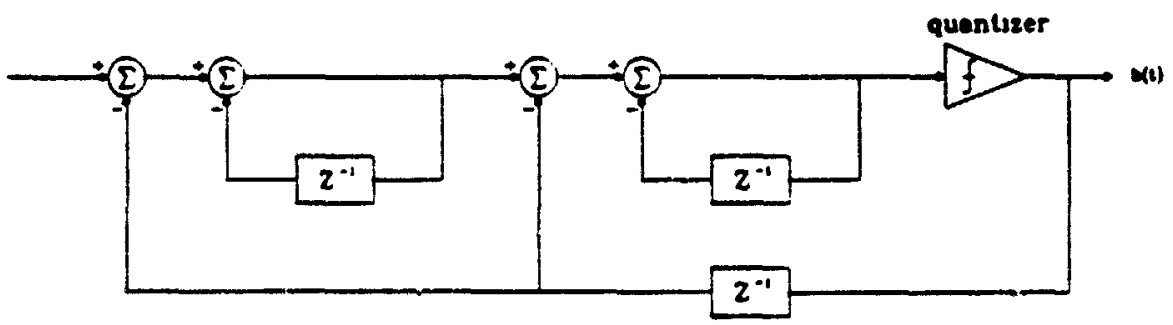

Figure 1.11: Second-Order Sigma-Delta Modulator 
behaviour of the circuit, based on its sampled-data and non-linear characteristics. In Chapter 3 we will consider these implications in terms of their effect on the frequency content of the VCO output. The conclusions derived from this discussion are validated by simulation results in Chapter 4 . The final chapter is devoted to a summary ania a survey of the possibilities for further investigation in this area. 


\section{Chapter 2}

\section{Modelling a Monolithic Frequency}

\section{Synthesizer}

\subsection{Introduction}

Generally, analysis of a frequency synthesizer takes the form common to continuous, feedback systems where a Laplace Transform is used to represent the time-domain behaviour of the system in the frequency-domain. This approach is appropriate when the time-averaged characteristics of the loop are of interest. However, we find that this method also tends to obscure the finer details of the behaviour of a loop intended for implementation as an integrated circuit and that these details can have a signif- 
icant impact on the output. In particular, we know that a fractional- $N$ frequency synthesizer of the kind described in the previous chapter is constantly undergoing time-domain transients as the divide ratio, $N$, is changed. Depending on the relative size of this change compared to the average value of $\mathrm{N}$, these transients may lead to unacceptable levels of phase noise at the output. As well, one of the main aims of this thesis is to determine how these transients interact with monolithic phase detectors. In both cases, we require more detail on the behaviour of the loop than can be obtained from a simple time-averaged analysis. As a result, in this chapter we develop an alternative representation of a monolithic frequency synthesizer.

Basically, we choose to consider the phase detector in a monolithic frequency synthesizer as a sampled-data element embedded in an otherwise continuous loop. The presence of a divider in the feedback loop of a frequency synthesizer implies that the feedback signal applied to the phase detector is rectangular. Then if the phase detector is to determine the phase difference between the reference signal and the feedback signal, it can only do so based on the edges of this signal. Conceivably we could filter the output of the divider to return it to a sinusoidal signal but because of the area constraints imposed by a monolithic implementation, this approach is not feasible. Even if it was possible to incorporate the filter on-chip, we would see that a continuous phase detector, such as a multiplier, requires more chip area than 
can be reasonably devoted to this function. As a result, a common characteristic shared by monolithic phase detectors is the fact that the output is determined by discrete events of the input signals, i.e. edges of a rectangular signal or zero-crossings of a sinusoid. Because of this discrete-time behaviour, one element of the model of a frequency synthesizer presented in this chapter is based on our view of the phase detector as sampling the phase difference between the reference and feedback signals. However, we will see that this form of sampling is significantly different than that :arried out by circuits like switched-capacitor filters; in fact, we have not attempted to establish the exact nature of this sampling since doing so raises questions which are beyond the scope of this thesis. Nevertheless, this view of the phase detector as sampling, in some manner, the continuous phase difference between Ref and Div is helpful in developing both an intuitive understanding of the behaviour of a monolithic frequency synthesizer and an explanation for certain elements of this behaviour not completely investigated elsewhere.

In order to extend this intuitive knowledge to include more quantitative characteristics, we have added a second element to the model of the frequency synthesizer, a time-domain representation of its behaviour. This representation has been tailored to a charge-pump phase detector-based implementation where both signals applied to the phase detector are rectangular. Then the form of the description of the fre- 
quency synthesizer is similar to that of a switched-capacitor filter; the quantity of interest at a certain time is expressed in terms of its earlier values. In the case of a frequency synthesizer we solve explicitly for the time between edges of the input and feedback signals. The benefit of this approach is that it is in no way based on a view of the phase detector as a sampled-data device. As a result, besides being a means of evaluating the characteristics of a particular frequency synthesizer, this model can also be used to validate conclusions drawn regarding the sampled-data nature of the phase detector.

\subsection{Monolithic Phase Detectors}

The specific details of this model vary depending on the characteristics of the phase detector. In addition to a charge-pump phase detector, there are two other circuits which are also commonly applied as integrated circuit phase detectors. The following sections describe the consequences for modelling a frequency synthesizer associated with all three circuits. 


\subsubsection{Charge-Pump Phase Detector}

A common configuration of a charge-pump phase detector is shown in Figure 2.1. The bi-directional charge-pump converts the dual outputs of the PFD into a current pulse through a continuous loop filter. The duration of the current pulse is determined by the difference in time between corresponding edges of the input signals, Ref and Div. If the Ref edge leads Div, indicative of a positive phase difference, the VCO frequency must be increased; the $U$ output is set with the Ref edge and reset by Div. Similarly, a Div edge occurring before Ref represents a negative phase error and causes a pulse on the D output and a current pulse at the output of the charge-pump in the opposite direction to the first case. The signals corresponding to both polarities of phase difference are shown in Figure 2.1b.

The dual-output nature of the PFD is difficult to model; for the sake of convenience we will combine the $U$ and $D$ signals into a single output $U / D$ and assume that the charge-pump is capable of inferring the correct polarity of the phase difference. Then, U/D will typically have the form shown in Figure 2.2 and we can begin to see the resemblance between U/D and a typical sampled-data signal. U/D consists of a train of pulses representing samples of the phase difference between Ref and Div. If we assume that the time between edges is small compared to the reference 


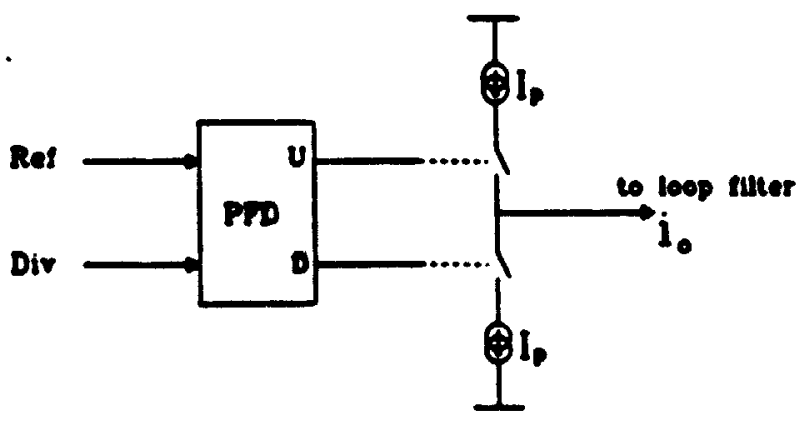

(a)
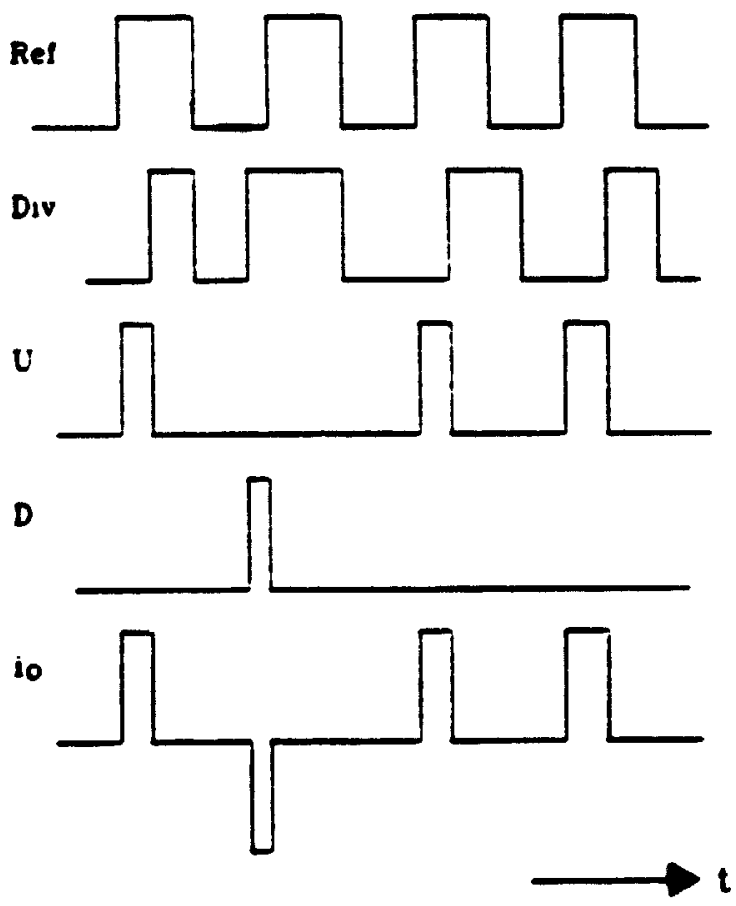

(b)

Figure 2.1: Charge-Pump Phase Detector (a) Schematic (b) Waveforms 
period, the pulses approach impulses and the resemblance to a sampled-data signal grows. However, the key difference between U/D and a signal commonly referred to as sampled-data is the fact the value of the sample is conveyed in the width of the pulse rather than its height. Nevertheless, it does seem reasonable to expect that the methods of analysis associated with circuits commonly referred to as sampled-data could also be applicable in the case of a charge-pump phase detector.

Analysis of a switched-capacitor filter is based on a single difference equation describing the value of the output at a certain time in terms of earlier values. Application of the z-transform and of the expression $z=\exp ^{j \omega t}$ gives the equivalent expression in the frequency domain. In the case of a frequency synthesizer we would like to write a single expression for $\Delta t$, the times difference between edges of Ref and Div, in terms of its earlier values i.e. $\Delta t[m]=f(\Delta t[m-1], \Delta t[m-2] \ldots)$. The relationship between the different values of $\Delta t$ is shown in Figure 2.2. After transforming to the frequency domain, it is a relatively straight-forward task to apply the transfer functions of the loop filter and VCO and we would be left with a single expression for the frequency content at the output of the VCO. Such an expression has the most value for assessing the performance of the circuit.

Unfortunately, the characteristics of a charge-pump phase detector make it difficult to write a single, closed-form expression for $\Delta t[m]$. The problem is simply that 


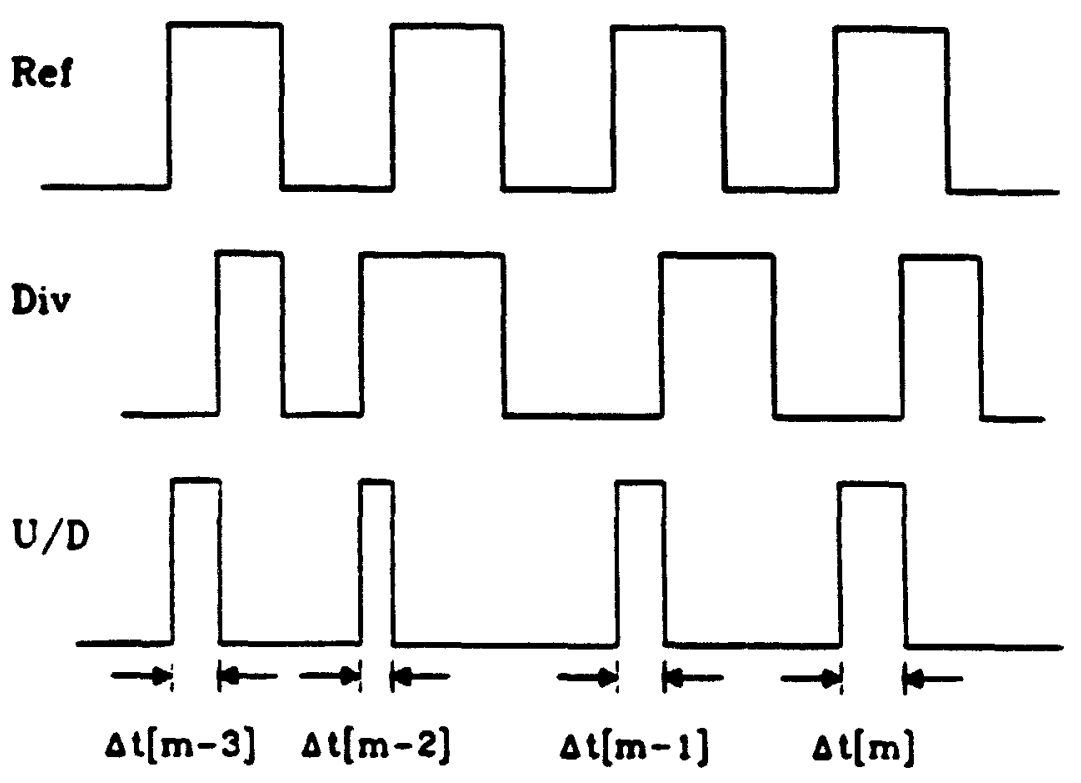

Figure 2.2: Dud Outputs of PFD Combined into a Single Signal

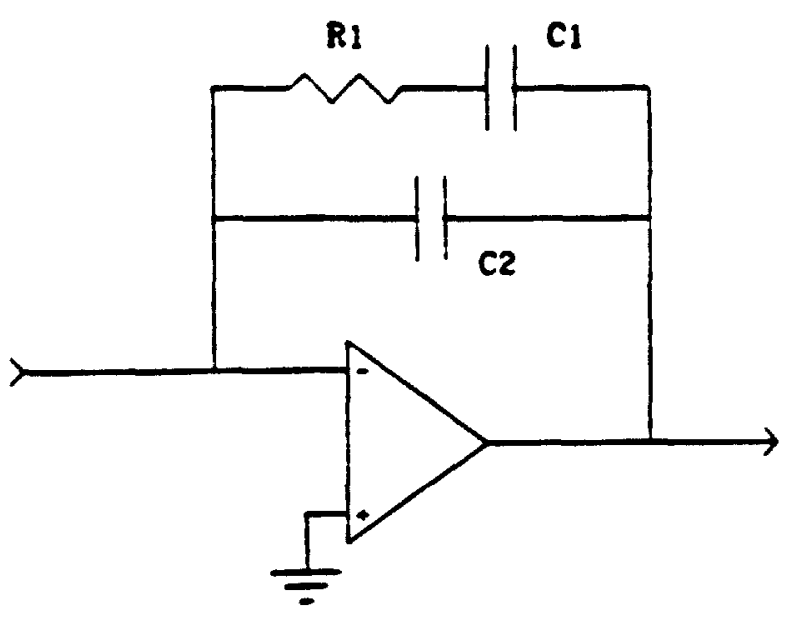

Figure 2.3: Lead-Lag Filter with Additional Pole 
the loop responds in a different manner depending on whether $\Delta t[m]$ is positive or negative. To see this consider the typical loop filter of Figure 2.3. The important point to note is that as soon as the charge-pump comes on and the transfer of charge through the loop filter begins, the output of the loop filter and the VCO output frequency immediately begin to change. In the case of a negative phase difference, where the Div edge occurs before Ref, the fact that the VCO frequency is changing during the time between edges has no effect on $\Delta t$. Conversely, when the phase difference is positive the charge-pump comes on with the Ref edge, causing the VCO frequency to change before the arrival of the Div edge. In other words, in order to write a single expression for $\Delta t[m]$ we must know beforehand whether $\Delta t[m]$ is positive or negative. Clearly, this is not possible for a typical loop where the phase difference between Ref and Div may be positive or negative. As a result, it appears as if we require two expressions for $\Delta t[m]$; in fact we have determined that in order to calculate $\Delta t[m]$, the polarity of $\Delta t[m-1]$ must also be considered. For the moment we will not attempt to provide proof of this statement; later when we consider this approach in more detail, its validity will be apparent. It is conceivable that we could attempt to find one expression for $\Delta t[m]$, representing the general behaviour of the loop, with non-linear terms included to account for the differences between positive and negative $\Delta t[m]$ but this approach is beyond the scope of this thesis. Instead, 
we can conclude that, in order to properly model a frequency synthesizer, we require four different expressions for $\Delta t[m]$ based on the possible combinations of polarities of $\Delta t[m]$ and $\Delta t[m-1]$. Then the basis of the model described in this chapter is not an analytical representation of a frequency synthesizer but rather a simulation of its time-domain behaviour. The Discrete Fourier Transform can then be used to transform a frame of this behaviour to the frequency domain.

As a final note in this discussion we should recognize that it is possible to isolate the VCO from the phase detector, and then write a single expres. on for $\Delta t[m]$, with a sample-and-hold circuit following the ioop filter. The sampling action could be synchronized to the unused edge of the Ref signal. However this has the effect of removing the filter resistor $R_{1}$ and the loop becomes unstable.

\subsubsection{Sampling Phase Detectors}

Before continuing with a discussion of the analytical racdel deycloped in this thesis, we can establish some context for a charge-pump phase detector-based frequency synthesizer by considering other circuits suitable for use as monolithic phase detectors. These circuits can be referred to collectively as sampling phase detectors because they are based on the sampling of an analog signal representing the phase difference 
between Ref and Div.

One type of sampling phase detector is shown in Figure 2.4a. A rising edge of the reference signal opens switch $S_{1}$ and initiates the discharge of a capacitor through a current source. [4] The corresponding edge from Div halts the discharge leaving a voltage on the ramp capacitor, $C_{r}$, as a measure of the phase difference. The falling edge of Ref can be used to momentarily close $S_{3}$ and sample this voltage onto the hold capacitor, $C_{h}$. Since linear operation of the phase detector requires that the Ref edges precede the edges from Div, we can assume that this is always the case. Then, at the output of the phase detector we would see a signal similar to that shown in Figure 2.4b.

In developing an analytical model of a loop en ploying this type of phase detector the important point to note is that, since we have assumed that the Ref edges always precede those of Div, the step changes in the output of the phase detector occur periodically. As a result, it seems that we could represent the behaviour of the loop with a single expression for $\Delta t$ in terms of earlier values. However, if the phase detector output is applied to a continuous loop filter, as is typical, the possibility exists that the output phase will change in the time between edges. If this is the case we would be unable to relate the values of $\Delta t$ to the phase difference between Ref and Div at a particular instant in time. making it difficult to accurately characterize 


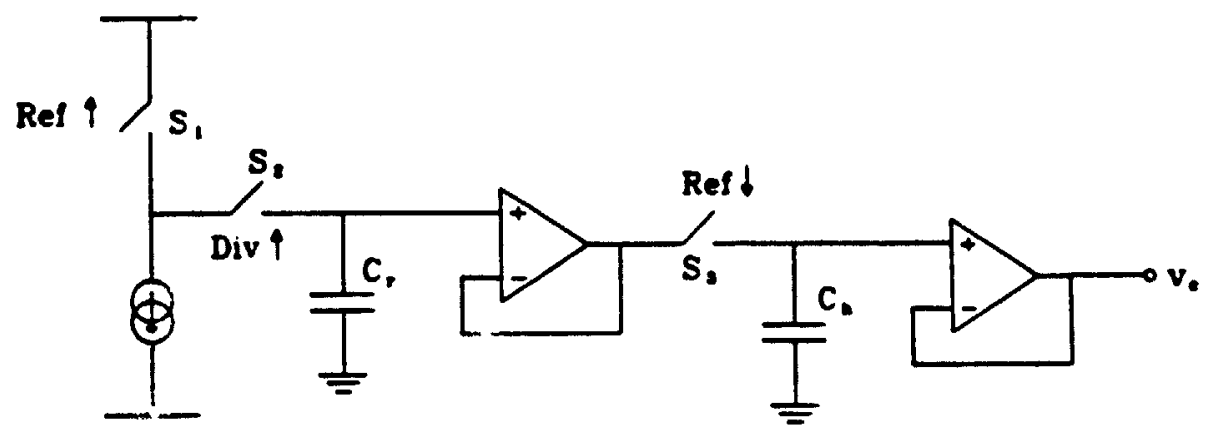

(a)

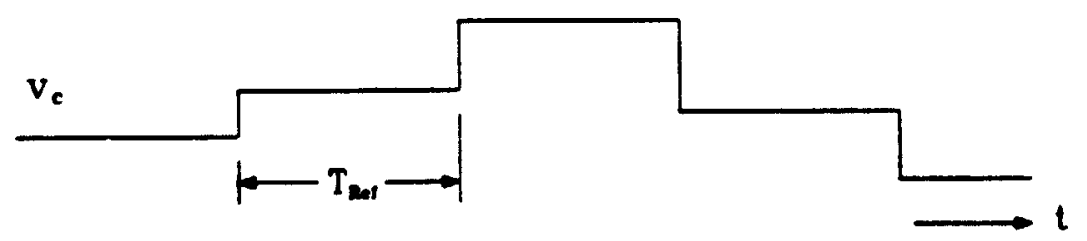

(b)

Figure 2.4: Sample-and-Hold Phase Detector a) Schematic b) Output the frequency domain behaviour of the loop.

Interchanging the functions of Ref and Div eliminates this problem as the discharge of the capacitor is initiated by a rising edge of Div and halted by a rising edge of Ref. If we are able to guarantee that the Div edges always precede those of Ref, then the time between edges is an accurate measure of the phase difference at the time of the Div edge and we should be able to develop the required frequency domain expression. 


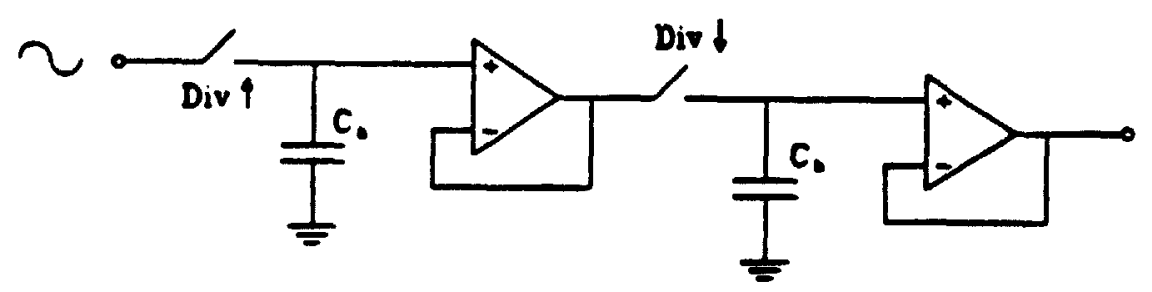

Figure 2.5: Sinsoidal Phase Detector

A similar phase detection technique uses a sinusoidal reference as shown in Figure 2.5. [5] The value of this signal at the time of the Div edge represents the phase difference. Although there is no explicit relationship between the phase difference and a time between edges, we can think of the sampled voltage as a measure of the time between the zero-crossing of the reference signal and the Div edge. It turns out that we can write a single expression for the frequency response of the loop provided we include non-linear terms to account for the nature of the sampling of the sinusoidal reference.

\subsubsection{Comparison}

From the preceding discussion it is clear that the charge-pump detector presents more significant problems for analysis than the sampling phase detectors. In its favour it can claim some operational advantages based on extended tracking range and frequency- 
aided acquisition. [6] The amount of charge transferred to the loop filter is a linear function of phase difference over the range $\pm 2 \pi$ radians. In comparison, sampling of a sinusoidal input signal leads to a sinusoidal phase detector characteristic with a period of $2 \pi$ radians. Furthermore, the sinusoidal characteristic leads to situations of positive feedback when the phase error samples are taken from the negatively sloping portion of the reference signal. As well, the requirement for a stable, low-distortion sinusoidal reference effectively ruled out the sinusoidal detector.

The ramping phase detectors are typically linear over a small fraction of $2 \pi$ radians and this, combined with the fact that they require that the edges of one input signal constantly precede those of the other for linear operation, was felt to be a limit to their applicability. Consequently, it was necessary to accept the problems for analysis posed by the charge-pump phase detector and to develop some means for their solution. This is the underlying theme of this thesis.

At this point it is possible to note a further benefit of the fractional- $\mathrm{N}$ approach to frequency synthesis. We have seen that many monolithic phase detectors make use of a current source to transfer charge to a capacitor. The relationship between the sizes of the current source and capacitor and the parameters of the overall loop can be demonstrated by considering a typical application. Assume frequencies in the range 300-500 $\mathrm{MHz}$ are to be synthesized from a $1 \mathrm{KHz}$ reference, while the VCO's 
input voltage range is $2 \mathrm{~V}$. The nominal value of the VCO gain, with the effect of the divider included for convenience, is

$$
\begin{aligned}
K_{0} & =\frac{\frac{500-300 \mathrm{MHz}}{2 \mathrm{~V}}}{400 * 10^{3}} \\
& =250 \mathrm{~Hz} / \mathrm{V} \\
& =500 \pi \mathrm{rad} / \mathrm{V}
\end{aligned}
$$

A rule-of-thumb[3] suggests that for those loops based on a charge-pump phase detector, the reference should be at least 10 times the loop bandwidth in order that overall behaviour is not significantly effected by the discrete-time characteristics. Therefore the loop bandwidth is $100 \mathrm{~Hz}$ and if 0.7 is used as the damping factor, from Chapter 1, the natural frequency is $143 \pi \mathrm{rads} / \mathrm{s}$. The natural frequency of the continuous approximation to the loop is given in the next section as

$$
\omega_{n}=\left(\frac{K_{0} I_{p}}{2 \pi C}\right)^{1 / 2}
$$

Rearranging gives the ratio of $I_{p}$ to $\mathrm{C}$ as

$$
\frac{I_{p}}{C}=\frac{2 \pi \omega_{n}^{2}}{K_{0}}
$$

Substituting the values of $K_{0}$ and $\omega_{n}$

$$
\frac{I_{p}}{C}=806
$$


With the maximum size of an on-chip capacitor limited to about $50 \mathrm{pF}$, the required value of $I_{p}$ is about $40 \mathrm{nA}$ and this is several orders of magnitude too small to be realized on the same device. However a fractional- $\mathrm{N}$ approach to frequency synthesis sees the loop bandwidth and natural frequency increase by possibly four or five orders of magnitude and the current source can then be reliably integrated.

\subsection{Closed-loop Analysis}

Now that we have established the basic characteristics of a charge-pump phase detector, we can turn our attention to the problem of accurately representing the behaviour of the loop. We have seen that a simulation structure devoted to charge-pump phase detector-based loops is the most reasonable approach for this thesis. One option would be to develop a simulator similar to SPICE where the change in the state of the loop is calculated over small increments of time. However this was viewed as introducing unnecessary complexity. The more general applicability was not warranted since the structure of the loop was well-known. Here we will consider another option based on an explicit solution for $\Delta t[m]$.

This method is similar to that presented by Gardner [6] but has been extended to allow the time-domain behaviour to be transformed to the frequency-domain. Ba- 
sically, Gardner divides the time-domain behaviour of the loop into intervals where the $m^{\text {th }}$ interval begins with the first edge of the $m^{\text {th }}$ pair of Ref and Div edges and ends with the first edge of the $m+1^{\text {th }}$ pair. (Figure 2.6) The actual edges which begin and end each interval could be due to either Ref or Div depending on the phase difference. Then a simple iterative approach is used to solve for the phase difference at the end of the $m^{\text {th }}$ interval in terms of the value from the previous interval. The actual duration of each interval depends on the polarity of the edge pairs.

In order to determine the frequency content of the VCO output, the state of the loop must be sampled throughout each interval rather than just at the end. This sampling is necessary during the time the charge-pump is on as well as off and requires the calculation of the actual time difference between edges instead of the phase difference at one edge. This calculation can be carried out iteratively and, once the value for the $m^{\text {th }}$ interval is found, samples of the instantaneous output frequency during the previous interval can be determined. An FFT on a large frame of these samples should give the desired spectral information. 


\subsubsection{Continuous-Time Equivalent}

Before considering the details of this approach, a useful relationship between $\omega_{n}$ and $\zeta$ of a continuous second-order loop and the elements of the sampled-data system can be developed if the time-averaged behaviour of the system is considered. [6] If $\boldsymbol{K}_{0}$ is the gain of the VCO, $I_{p}$ is the value of the current source, and $\mathrm{R}$ and $C_{1}$ are as shown in Figure 2.3 this relationship can be summarized as

$$
\begin{aligned}
\tau_{1} & =R_{1} C_{1} \\
\omega_{n} & =\left(\frac{K_{o} I_{p}}{2 \pi C_{1}}\right)^{1 / 2} \\
\zeta & =\frac{\tau_{1}}{2}\left[\frac{K_{0} I_{p}}{2 \pi C_{1}}\right]^{1 / 2} \\
K & =\frac{K_{o} I_{p} R_{1}}{2 \pi}
\end{aligned}
$$

where $\mathrm{K}$ is the unity-gain bandwidth of the open-loop transfer function. These equations make it possible to calculate required values of $K_{o}, I_{p}, R_{1}$, and $C_{1}$ from the specification of the loop in terms of continuous, second-order parameters. Note that these relationships are based on the low frequency behaviour of the loop. As a result, the effect of $C_{2}$ has been neglected. 


\subsubsection{Time-Domain Representation}

Moving now to the time-domain behaviour of the loop, the important point for modelling this behaviour is that, if $t_{0}$ is the time between edges of the divider output, it can be evaluated as the time required for a change of $2 N \pi$ radians in the VCO output.

$$
2 N \pi=\omega_{0} t_{0}+K_{v} \int_{0}^{t_{0}} v_{c}(t) d t
$$

Then $\Delta t[m]$ can be found from

$$
\Delta t[m]=\Delta t[m-1]+t_{0}-T
$$

where $T$ is the reference signal period. Consequently, a simulation of the loop simply requires that we be able to determine $t_{0}$ for any values of $\Delta t[m]$ and $\Delta t[m-1]$.

Consider the evaluation of $\Delta t[m]$ when $\Delta t[m-1]$ is positive, i.e. the $m-1^{\text {th }}$ Ref edge precedes that of Div. As shown in Figure 2.6a, if $\Delta t[m]$ is negative, the next edge of Div occurs before its Ref counterpart and the charge-pump remains off for the entire length of $t_{0}$. Then $v_{c}(t)$ from Equation 2.5 can be described as a decaying exponential with its initial value determined during the calculations for the previous interval. However, if $\Delta t[m]$ is positive, calculation of its value must account for the charge-pump being on during the final portion of $t_{0}$, as shown in Figure 2.6b. Much the same argument can be applied when $\Delta t[m-1]$ is negative. In this case, $\Delta t[m]$ 
is found assuming it is also negative but the calculation must account for the chargepump being on during the first part of $t_{0}$. If this calculation shows that $\Delta t[m]$ is, in fact, positive, it is repeated but includes the effect of the charge-pump coming on before the arrival on the $m^{\text {th }}$ edge of Div.

It is clear that instead of a single closed-form expression for $\Delta t[m]$, there are four different combinations of $\Delta t[m]$ and $\Delta t[m-1]$, depending on the individual polarities, and each involves a different form of the integration of $v_{c}(t)$ of Equation 2.5 . Then analysis of the time-domain behaviour of the loop must first determine whether $\Delta t[m-1]$ is positive or negative and follow this with an appropriate calculation of $\Delta t[m]$ assuming it to be negative. If the result is positive the calculation is repeated to account for the effect of the charge-pump. Given below is a detailed description of this process when both $\Delta t[m-1]$ and $\Delta t[m]$ are negative.

When the $(m-1)^{\text {th }}$ edge of Div arrives, it causes the charge-pump to come on and draw a current $I_{p}$ through the feedback network of the loop filter and the change in $v_{c}(t)$ is as shown in Figure 2.7. The assumption of the direction of the current is based on the chosen reference, i.e. a positive time difference implies a current into the inverting node. Until the edge of Ref arrives, $v_{c}(t)$ is given by

$$
v_{c}(t)=v_{c}[m-2]-\frac{i_{1}[m-2] t}{C_{2}}-\frac{I_{p} t}{C_{1}+C_{2}}+I_{p} R\left(\frac{C_{1}}{C_{1}+C_{2}}\right)^{2}\left[\exp \left(\frac{-t}{R C_{\text {eq }}}\right)-1\right]
$$




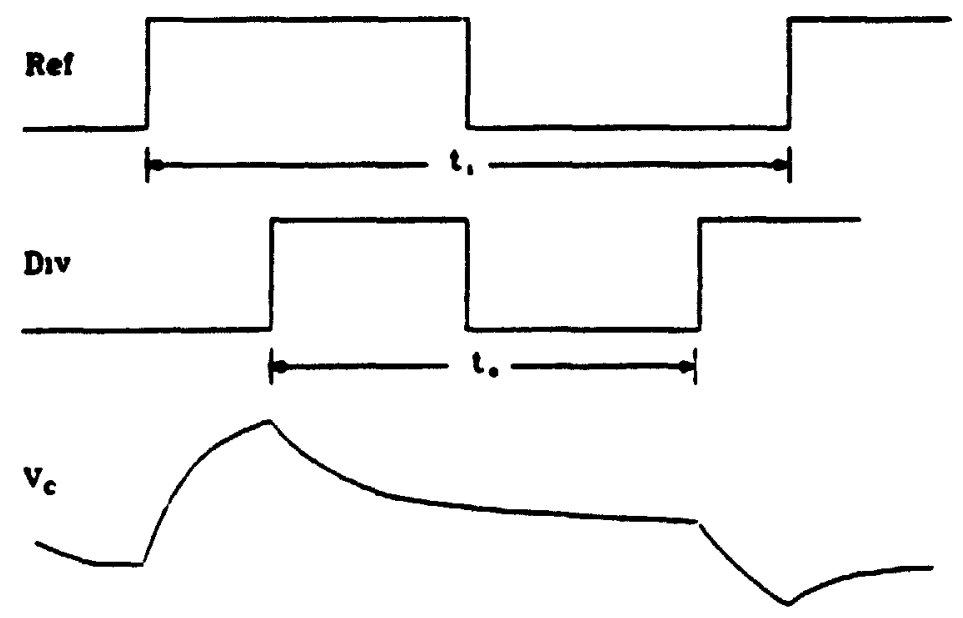

(a)

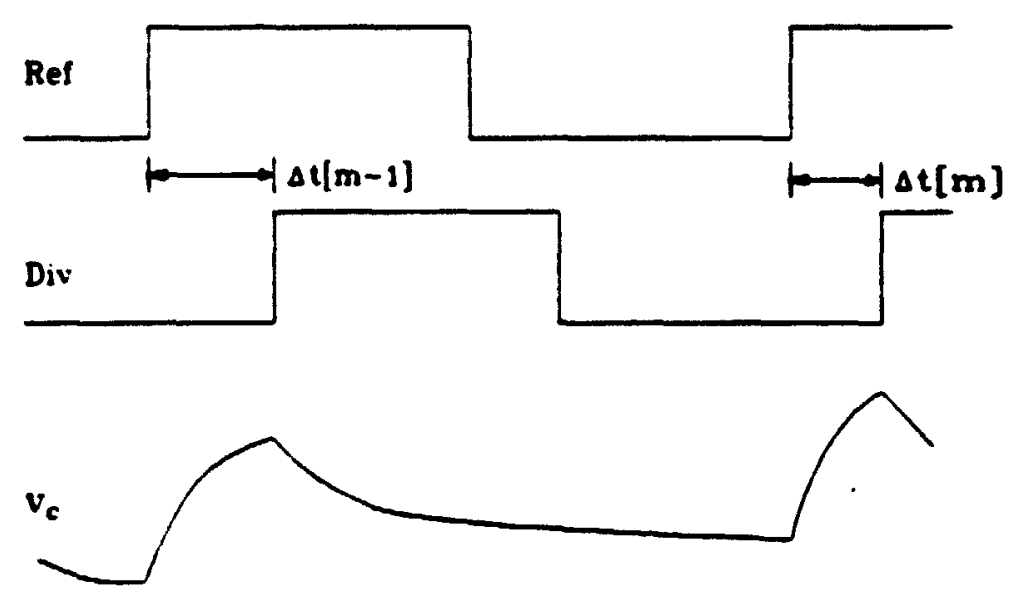

(b)

Figure 2.6: Examples of Time-Domain Behaviour 


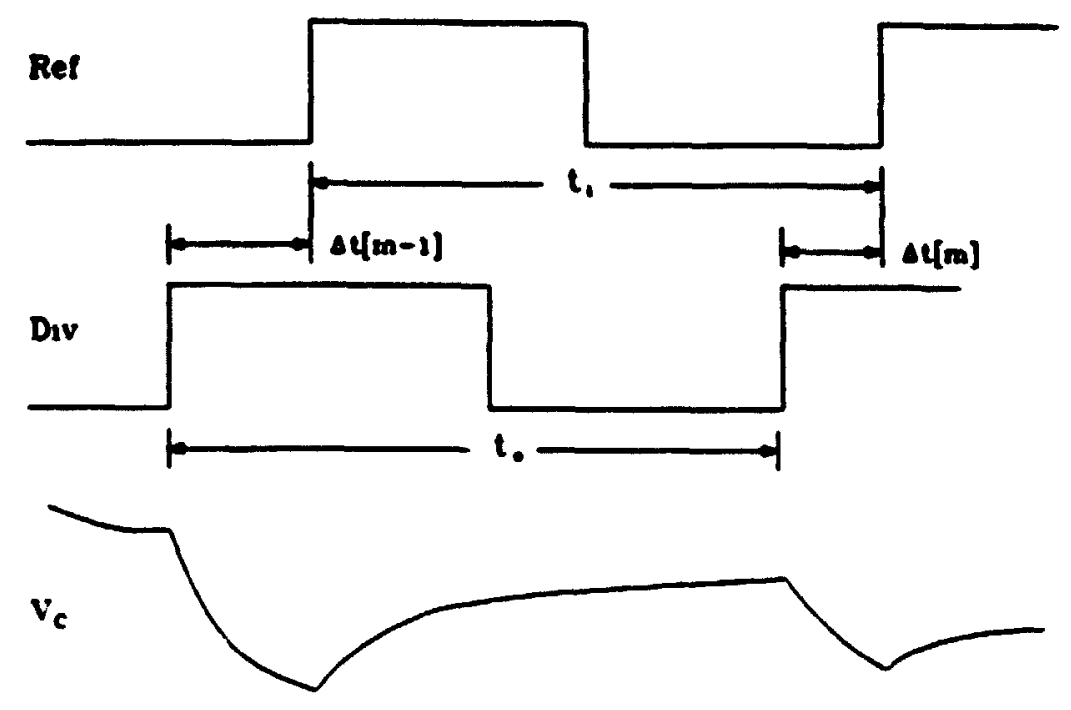

Figure 2.7: $V_{c}(t)$ for Negative $\Delta t[m-1]$ and $\Delta t[m]$

and the current through the resistor, $R_{1}$, is

$$
i_{1}(t)=i_{1}[m-2]+\frac{I_{p} C_{1}}{C_{1}+C_{2}}\left(\exp \left(\frac{-t}{R C_{e q}}\right)-1\right)
$$

where $v_{c}[m-2]$ and $i_{1}[m-2]$ correspond to the voltage and current at the end of the $m-2^{\text {th }}$ interval.

The integral of Equation 2.5 can be evaluated in two parts, one over $\Delta t[m-1]$ and the other over the remainder of $t_{0}$.

$$
2 N \pi=\omega_{0} t_{0}+K_{v}\left(\int_{0}^{\Delta t[m-1]} v_{c}(t) d t+\int_{\Delta t[m-1]}^{t_{0}} v_{c}(t) d t\right)
$$


The first integral gives the change in output phase over $\Delta t[m-1]$ as

$$
\begin{aligned}
\dot{\theta}_{0}[n-1]= & K_{v}\left(\int_{0}^{\Delta t[m-1]} v_{c}(t) d t\right) \\
= & K_{v}\left[\frac { - I _ { p } } { C _ { 1 } ( C _ { 1 } + C _ { 2 } ) } \left[\frac{1}{2} C_{2} \Delta t[m-1]^{2}\right.\right. \\
& \left.+\left(R C_{e q}\right)^{2} C_{1}\left(\exp \left(\frac{-\Delta t[m-1]}{R C_{e q}}\right)-1\right)\right] \\
& -\frac{i_{1}[m-2] \Delta t[m-1]^{2}}{2 C_{2}} \\
& \left.-I_{p} R\left(\frac{C_{1}}{C_{1}+C_{2}}\right) \Delta t[m-1]+v_{c}[m-2] \Delta t[m-1]\right]
\end{aligned}
$$

When the charge-pump is off

$$
\begin{gathered}
v_{c}=\hat{v}_{e}[m-1]+\hat{i}_{1}[m-1] R\left(\frac{C_{1}}{C_{1}+C_{2}}\left[\exp \left(\frac{-t}{R C e q}\right)-1\right]\right. \\
i_{1}(t)=\hat{i}_{1}[m-1] \exp \left(\frac{-t}{R C e q}\right)
\end{gathered}
$$

Here, the values of $\dot{v}_{c}[m-1]$ and $\dot{i}_{1}[m-1]$ refer to the values of voltage and current the instant the current is removed.

Equation 2.10 can be used to evaluate the second integral of Equation 2.9 which then becomes

$$
\begin{aligned}
2 N \pi= & \omega_{0} t_{0}+\dot{\theta}_{0}[m-1]+K_{0}\left[\hat{v}_{c}[m-1]\left(t_{0}-\Delta t[m-1]\right)\right. \\
& -\hat{i}_{1}[m-1] \frac{\left(R C_{\text {eq }}\right)^{2}}{C_{2}}\left[\exp \left(\frac{-\Delta t[m-1]}{R C_{\text {eq }}}\right)\left(\exp \left(\frac{-\left(t_{0}-\Delta t[m-1]\right)}{R C_{\text {eq }}}\right)-1\right)\right. \\
& \left.\left.+t_{0}-\Delta t[m-1]\right]\right]
\end{aligned}
$$


It is not possible to explicitly solve Equation $2.1 \%$ for $t_{o}$ so an iterative process must be used. Basically, starting with two values of $t_{0}$ which are sure to be greater and less than the true value respectively, their average is substituted into the right. hand-sice of Equation 2.12 and the result compared witi $2 N \pi$. If the difference is less than some specified tolerance the calculation of $t_{0}$ is complete; otherwise that value of $t_{0}$ replaces one of the boundary values and the process is repeated. Once $t_{0}$ is found, equation 2.6 is used to find $\Delta t[m]$ and values of $v_{c}[m-1]$ and $i_{1}[m-1]$ are calculated for use during the next iteration. A similar development is required with the other three combinations of polarities of $\Delta t[m]$ and $\Delta t[m-1]$.

It is important to note the limitations of this model of a frequency synthesizer. Because it is strictly a time-domain representation of the behaviour of the loop, any input must also be characterized in the time domain. In other words we can only consider the effects of a noisy reference or VCO if that noise can be modelled in the time domain. Generally this is not easy for an arbitrary noise spectrum.

\subsubsection{Frequency-Domain Representation}

A Discrete Fourier Transform is the obvious choice for representing a segment of the time-domain behaviour of the loop in the frequency domain. If we want the results 
of the DFT to resemble what we would see if a spectrum analyzer was placed on the output of the VCO, the samples on which the DFT is performed must be of the signal amplitude. Then, we would expect the spectrum to contain a dominant component at the desired frequelsy along with a two-sided 'noise' spectrum showing the effects of the charge-pump phase detector and the dual-modulus divider. However it was found that because of the nature of the simulation model, it is easier to perform the DFT on samples of the instantaneous output frequency, $\omega_{\text {ins }}(t)$. In this way an additional calculation of signal amplitude from frequency is eliminated. Nevertheless, if we are to make any evaluation of the effect of the noise on the output, we must be able to relate the results of the DFT to something which is more intuitively meaningful, i.e. the spectrum of the true VCO output signal.

Fortunately, the spectra of the amplitude of the VCO output and of its instantaneous frequency can be related in the following manner. We will consider the output as a sinusoidal signal with a main frequency of $\omega_{c}$ and also a phase noise $n(t)$.

$$
y(t)=A \cos \left(\omega_{c} t+n(t)\right)
$$

A spectrum analyzer would show a component at $\omega_{c}$ as well as the two-sided phase noise spectrum, $S_{n}(\omega)$, centered on $\omega_{c}$. The instantaneous phase of $y(t)$ is

$$
\theta_{\text {sno }}(t)=\omega_{c} t+n(t)
$$


and the instantaneous frequency is

$$
\begin{aligned}
\omega_{\text {ina }}(t) & =\frac{d}{d t}\left(\theta_{\text {ins }}(t)\right) \\
& =\omega_{c}+\frac{d}{d t}(n(t))
\end{aligned}
$$

Evidently a DFT on samples of $\omega_{\mathrm{in}}(t)$ will show a component at dc whose magnitude is determined by $\omega_{c}$ and the spectrum corresponding to $\frac{d}{d t}[n(t)]$. If we recall a fundamental Laplace transform relationship,

$$
\mathcal{L}\left(\frac{d}{d t} n(t)\right)=s N(s)
$$

we can see that, besides the component at dc, the DFT will show the spectrum of the original phase noise, $S_{n}(\omega)$, scaled at a rate of $20 \mathrm{db} / \mathrm{dec}$. Recalling that the theoretical shaping of the quantization noise associated with a second-order sigmadelta modulator is $40 \mathrm{db} / \mathrm{dec}$, we would expect the spectrum of the instantancous output frequency to show a shaping of $60 \mathrm{db} / \mathrm{dec}$. Of course, the actual level of quantization noise also depends on the low-pass filtering action of the forward path of the loop. With these points in mind, we should be able to use the simulation results to judge the stability of the output frequency. 


\subsection{Summary}

In this chapter we have presented a model to be used in the evaluation of the behaviour of a fractional- $N$ frequency synthesizer employing a charge-pump phase detector. Based on our view of the phase detector as a sampled-data device, originally it was hoped that this model would take the form of a single expression for the frequency response of the loop. However, we found that, because of the nature of the charge-pump phase detector, development of this expression was beyond the scope of this thesis. Instead, we have resorted to a time-domain simulation of the loop and this simulation structure represents one of the main achievements of this thesis. The benefit of this approach is that it can be used to investigate the other main area of our interest, i.e. the consequences of the sampled-data and non-linear characteristics of the phase detector. In the next chapter, we will see that these consequences must be considered as a potential source of error in the output of the loop. 


\section{Chapter 3}

\section{Sampled-Data and Non-Linear}

\section{Aspects}

\subsection{Introduction}

Recall that the purpose of this thesis is the examination of the time-domain and frequency-domain behaviour of a fractional- $\mathrm{N}$ frequency synthesizer. In this chapter, we will focus on the aspects of this behaviour which are related to a monolithic implementation of the lomp. Specifically, we will consider the relationship betwerell the sampled-data and non-linear characteristics of a charge-pump phase detector and the phase noise content of the output of the VCO. 
Previously, we introduced the idea of a monolithic phase detector as a sampleddata element. Because of the way in which it responds to discrete events at its inputs, i.e. zero-crossings or edges, we found it appropriate to think of the phase detector as sampling the phase difference between the input and feedback signals in much the same way that a switched-capacitor filter samples its input voltage. In a typical context, we know that the act of sampling a signal creates replicas of the spectrum of that signal around all multiples of the sampling frequency. When the bandwidth of the original signal is large, portions of these replicas may fall within the baseband range of frequencies, a phenomenon known as aliasing. The term, aliasing, refers to the fact that when this occurs, the true nature of the signal is lost, or aliased. We believe that such spectral replication is also implied by the discrete-time nature of monolithic phase detectors. In other words, we believe that the output of the phase detector contains replicas of $\theta_{d}(\omega)$, the spectrum of the phase difference between the reference and feedback signals, around all multiples of the reference frequency, $f_{i}$. In an ideal phase-locked loop the phase difference is zero but, in practice, $\theta_{d}(\omega)$ will have a distributed spectral density due to thermal noise, VCO phase noise, coupled power supply noise, etc.. We will see that wideband components of $\theta_{d}(\omega)$, which would have little effect on the VCO output of a loop with a mulitplier as the phase detertor, can be replicated near dc when the multiplier is replaced by a charge-pump 
phase detector. Those components replicated within the loop bandwidth are expected to bring about an increase in phase noise around the average frequency of the output and a degradation from the baseband noise-shaping characteristics displayed by the sigma-delta approach to fractional-N synthesis. Consequent!y, one aim of this chapter is to develop some insight into the effect of the replicas of $\theta_{d}(\omega)$ on the VCO output.

The non-linear nature of typical monolithic charge-pump phase detectors is also of interest, again, because of the possibility of increased phase noise near the average VCO output frequency. Generally, we know that a non-linearity is capable of generating spurious spectral components and that intermodulation of the input spectrum may see some of these spurious components fall at or near dc. As a result, a non-linear phase detector may lead directly to an increase in output phase noise. However, it is also possible that, when a charge-pump phase detector is used, some of the higher frequency components generated by the non-linearity will be replicated to within the loop bandwidth. We will attempt to determine if intermodulation and/or spectral replication is implied by the common causes of a non-linearity in a charge-pump phase detector. 


\subsection{Sampling and Spectral Repication}

Replication of $\theta_{d}(\omega)$ by a charge-pump phase detector, if actually the case, represents an important difference between tbe loop under discussion here and one using a multiplier as a phase detector. Consequently, we will use the intuitive argument below, followed by a more rigorous approach, to show that these replicas are part of the output of the phase detector. Consider a sinusoidal reference signal

$$
\operatorname{Re} f=A \sin \left(\omega_{i} t\right)
$$

and a feedback signal whose phase includes additive noise, $\theta_{n}(t)$.

$$
\operatorname{Div}=A \sin \left(\omega_{\mathrm{r}} t+\theta_{n}(t)\right)
$$

For simplicity, we will restrict $\theta_{n}(t)$ to a single frequency, $\omega_{\theta_{n}}$ of magnitude $B$, and Equation 3.2 becomes

$$
D_{i v}=A \sin \left(\omega_{i} t+B \sin \omega_{\theta_{n}} t\right)
$$

Then the phase difference between Ref and Div is

$$
\theta_{d}(t)=-B \sin \omega_{\theta_{n}} t
$$

Generally, if $\omega_{\theta_{n}}$ is greater than the loop bandwidth, the phase noise has little effect on the response of the loop. However, this does not account for the fact that monolithic phase detectors measure $\theta_{d}(t)$ according to discrete events in Ref and Div. 


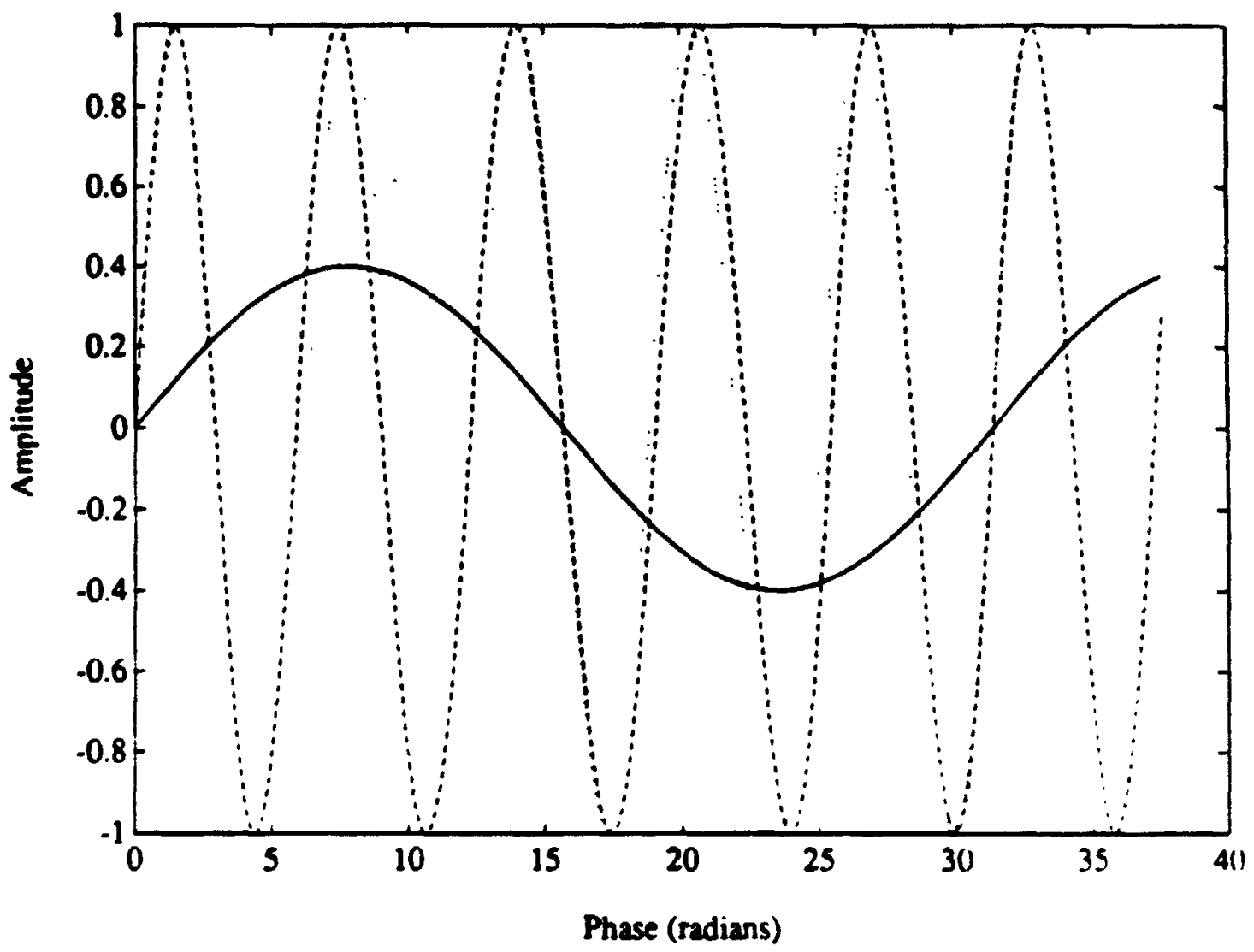

Figure 3.1: Ref ..., Div.,- and $\theta_{d}(t) \longrightarrow$ when $B=0.4$ and w $\omega_{n}=0.2 * \omega_{\text {, }}$

Consider a case where $\omega_{0_{n}}$ is much smaller than $\omega_{i}$; Figure 3.1 shows plots of Ref, Div, and $\theta_{d}(t)$ when $B=0.4$ and $\omega_{0 n}=\frac{1}{3} \omega_{1}$. Ref is represented by the dotted line, Div by the dashed line, and $\theta_{d}(t)$ by the solid line.It is clear that, if we think of the times between zero-crossings of Ref and Div as samples of $\theta_{d}(t)$, these samples accurately convey the true nature of the continuous phase difference. However, this is not the case when $\omega_{0 .}$ is, say, slightly greater than $\omega_{1}$. In Figure 3.2 we can see that, if we 
consider only the zero-crossings of Ref and Div, the fundamental component of $\theta_{d}(t)$ is at a frequency which is much smaller than $\omega_{o_{n}}$. In other words, the true nature of $\theta_{d}$ has been aliased due to replication of portions of $\theta_{d}(\omega)$ within the baseband range of frequencies. This would seem to be of major significance. However, several points should be noted. First, in Chapter 2, we saw that the discrete-time characteristics of monolithic phase detectors are significantly different from those of typical sampleddata circuits. The main distinction was in the representation of the samples of $\theta_{d}(t)$ in terms of the widths of the output pulses rather than their amplitudes. We should also be aware that the phase detector is sampling a quantity, $\theta_{d}(t)$, which is not explicitly represented by a physical signal. As a result, the preceding conclusion regarding replication of $\theta_{d}(\omega)$ must be viewed with some degree of caution unless we can develop a more rigorous proof of its validity. Fortunately, this is possible, to a cert ain extent, with an approach similar to that used to characterize the output of the typical sampling stage shown in Figure 3.3a. This approach is well-known [7] but is described here in some detail, for reference. 


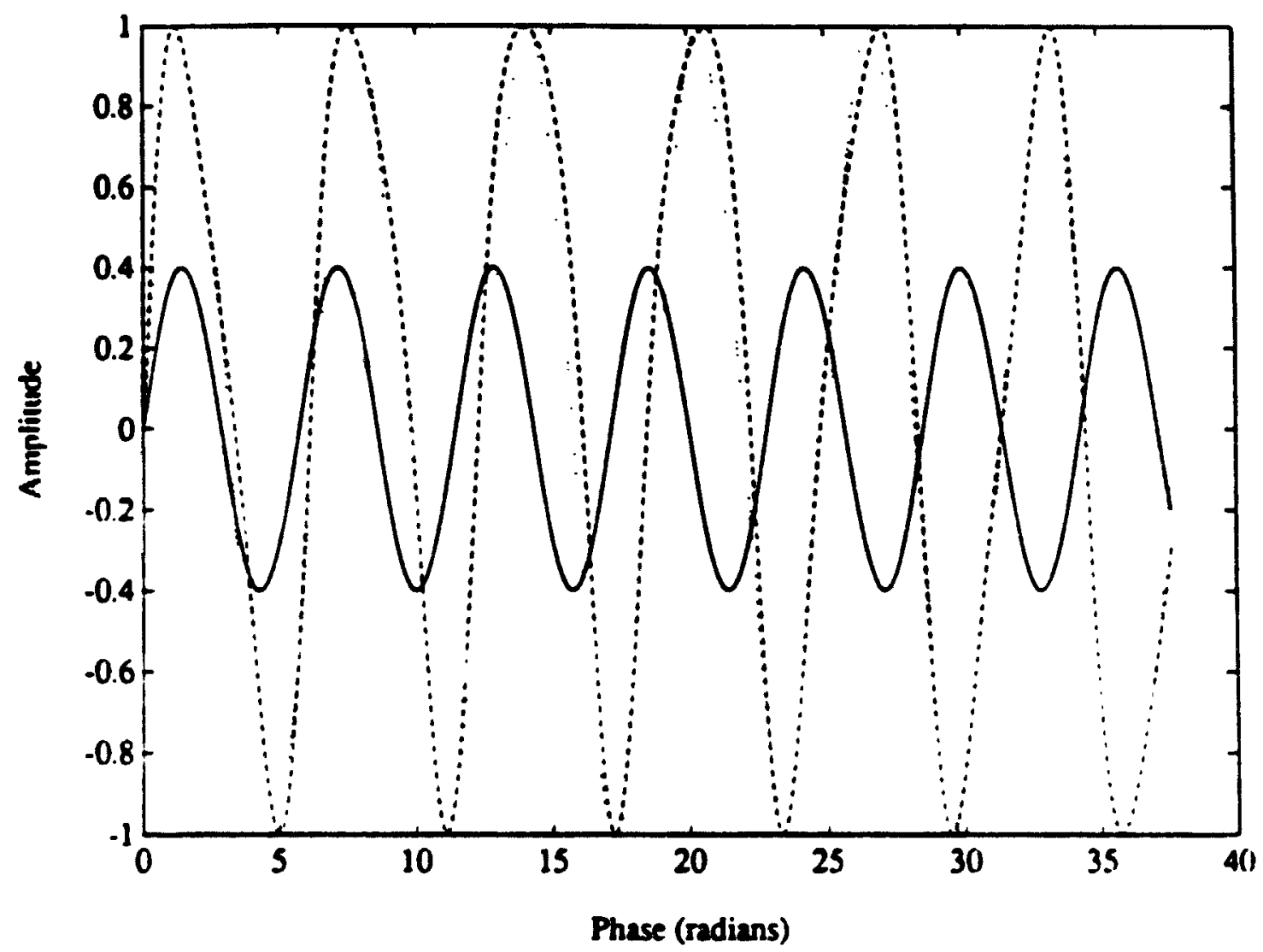

Figure 3.2: Ref $\ldots$, Div $\cdots$, and $\theta_{d}(t) \longrightarrow$ when $B=0.4$ and $\omega_{0 .}=1.1 * u_{\text {i }}$

\subsubsection{Sampling and Replication in a Typical Context}

The output of the sampling stage, $f^{\circ}(t)$, consists of a train of variable beight pulses with a fixed width, $\delta$, as shown in Figure 3.3b. The pulses can be defined in terms of step functions as [7]

$$
f^{\circ}(t)=K \sum_{n=0}^{\infty} f(n T)[u(t-n T)-u(t-n T-\delta)]
$$




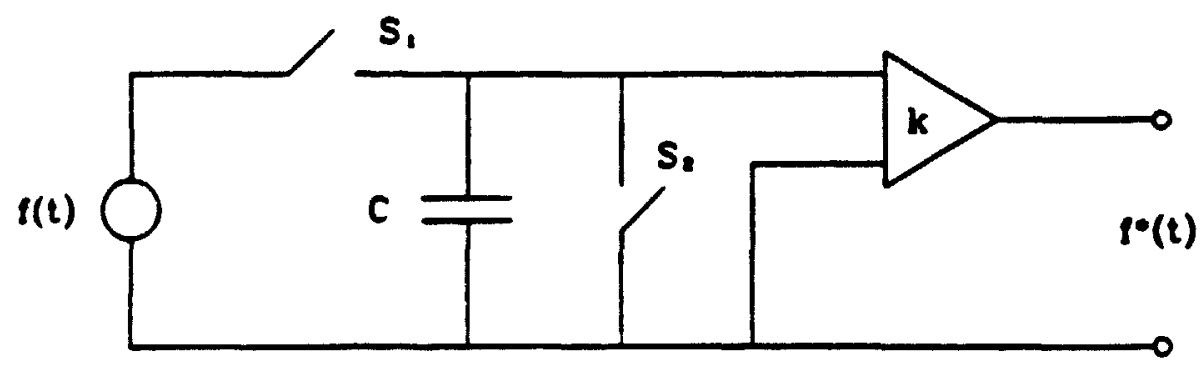

(a)

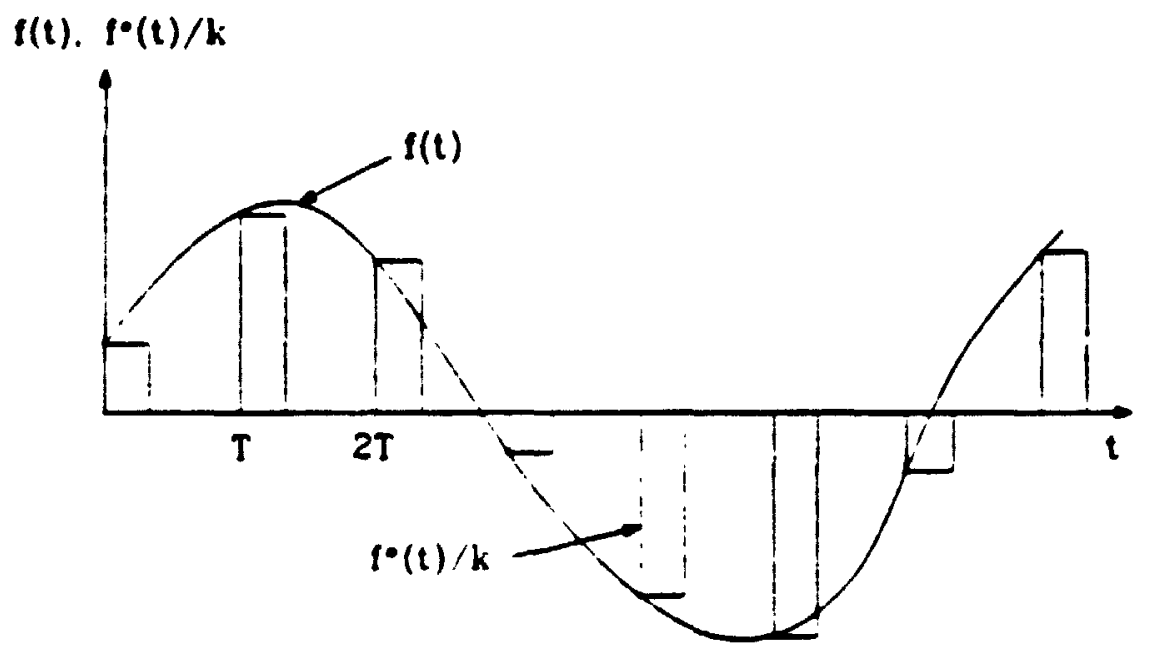

( $($ )

Figure 3.3: Sampling Stage (a) Schematic (b) Waveforms 
where $T$ is the sampling period. The Laplace transform of $f^{-}(t)$ is

$$
F^{*}(s)=K \frac{1-\exp ^{-\infty}}{s} \sum_{n=0}^{\infty} f(n T) \exp ^{-a n T}
$$

The spectrum of the output signal is obtained by setting $s=j \omega$ in Equation 3.6

$$
\begin{aligned}
F^{*}(j \omega) & =K \frac{1-\exp ^{-j \omega \delta}}{j \omega} \sum_{n=0}^{\infty} f(n T) \exp ^{-j \omega n T} \\
& =K^{\prime} \delta \exp ^{-j \omega \frac{\delta}{2}}\left(\frac{\sin \omega \frac{\delta}{2}}{\omega \frac{\delta}{2}}\right) \sum_{n=0}^{\infty} f(n T) \exp ^{-j \omega n T}
\end{aligned}
$$

If $K$ is chosen as $\frac{1}{6}$, Equation 3.7 becomes

$$
F^{*}(j \omega)=\exp ^{-\jmath \omega \frac{t}{2}}\left(\frac{\sin \omega \frac{\delta}{2}}{\omega \frac{\delta}{2}}\right) \sum_{n=0}^{\infty} f(n T) \exp ^{-\jmath \omega n T}
$$

Using some basic relationships of the Fourier transformation, Equation 3.8 can be rewritten as

$$
F^{*}(j \omega)=\exp ^{-j \omega \frac{f}{2}}\left(\frac{\sin \omega \frac{\delta}{2}}{\omega \frac{6}{2}}\right) \sum_{k=-\infty}^{\infty} F(j \omega-j k 2 \pi / T)
$$

Equation 3.9 indicates that $F^{\circ}(j \omega)$ consists of replicas of the input spectrum, scaled by the $\frac{\sin x}{x}$ factor due to the finite width of the samples, around all multiples of the sampling frequency, $f_{0}=\frac{1}{T}$. Note that when the bandwidth of the input signal is greater than $\frac{6}{2}$, portions of the spectral replicas fall within the baseband range of frequencies and the fundamental nature of the input signal is lost or aliased.

The effect of this aliasi.ıg has been demonstrated for the simple sample-and-hold circuit shown in Figure 3.4. The thermal noise of the switch resistance possesses a 


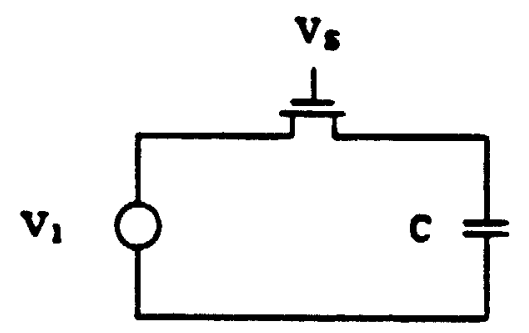

(a)

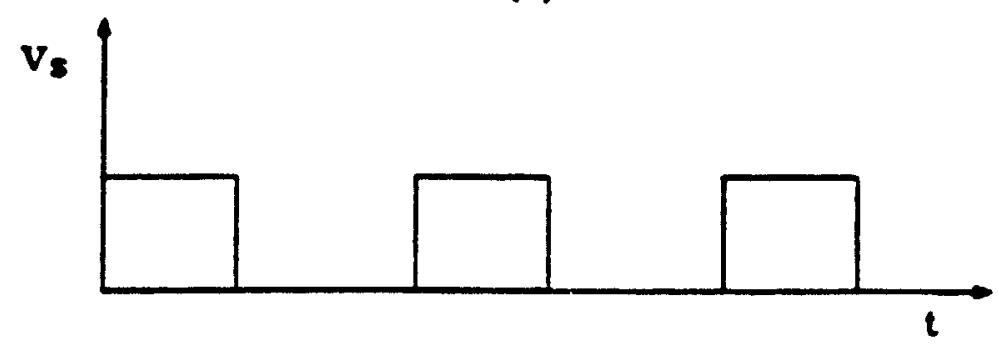

(b)

Figure 3.4: Basic Sample-and-Hold Circuit

bandwidth which is typically large compared to the sampling frequency. The result is a concentration within the baseband of the full noise power of the switch resistance.[7]

\subsubsection{Sampling by a Phase Detector}

Now consider sampling by monolithic phase detectors. In order to simplify this discussion, we will consider only a charge-pump phase detector although the conclusions drawn here are equally applicable to the other phase detectors described earlier. In Chapter 2 we found it useful to think of the phase/frequency detector in a charge- 
pump phase detector as a single output device whose output signal consists of fixed amplitude, variable width pulses representing samples of $\theta_{d}(t)$. Then, it seems plausible that we could use the approach of the previous section to describe the output of the phase detector and thereby confirm that $\theta_{d}(\omega)$ is replicated due to sampling by the phase detector. Consider Figure 3.5 which shows a continuous $\theta_{d}(t)$ and the output of the phase detector, $\theta_{d}^{\prime}(t)$, as pulses of fixed amplitude $A$ and variable width $\delta_{n}$. For convenience, we will assume that the phase detector is not part of a closed loop, When the $n^{\text {th }}$ pulse represents a positive phase difference, we can describe $\theta_{d}^{*}(t)$ as

$$
\theta_{d}^{*}(t)=A\left[u(t-n T)-u\left(t-n T-\delta_{n}\right)\right]
$$

where $T$ is period of the reference signal. The width of the pulse is determined by phase difference at the time of the first edge

$$
\delta_{n}=k \theta_{d}(n T)
$$

where $k$ is a constant of proportionality. Then, Equation 3.10 becomes

$$
\theta_{d}^{*}(t)=A\left[u(t-n T)-u\left(t-n T-\delta_{n}\right)\right]
$$

If $\theta_{d}(t)$ was always positive, Equation 3.12 could be used to develop an expression for the frequency content of the phase detector output. However, to be completely 


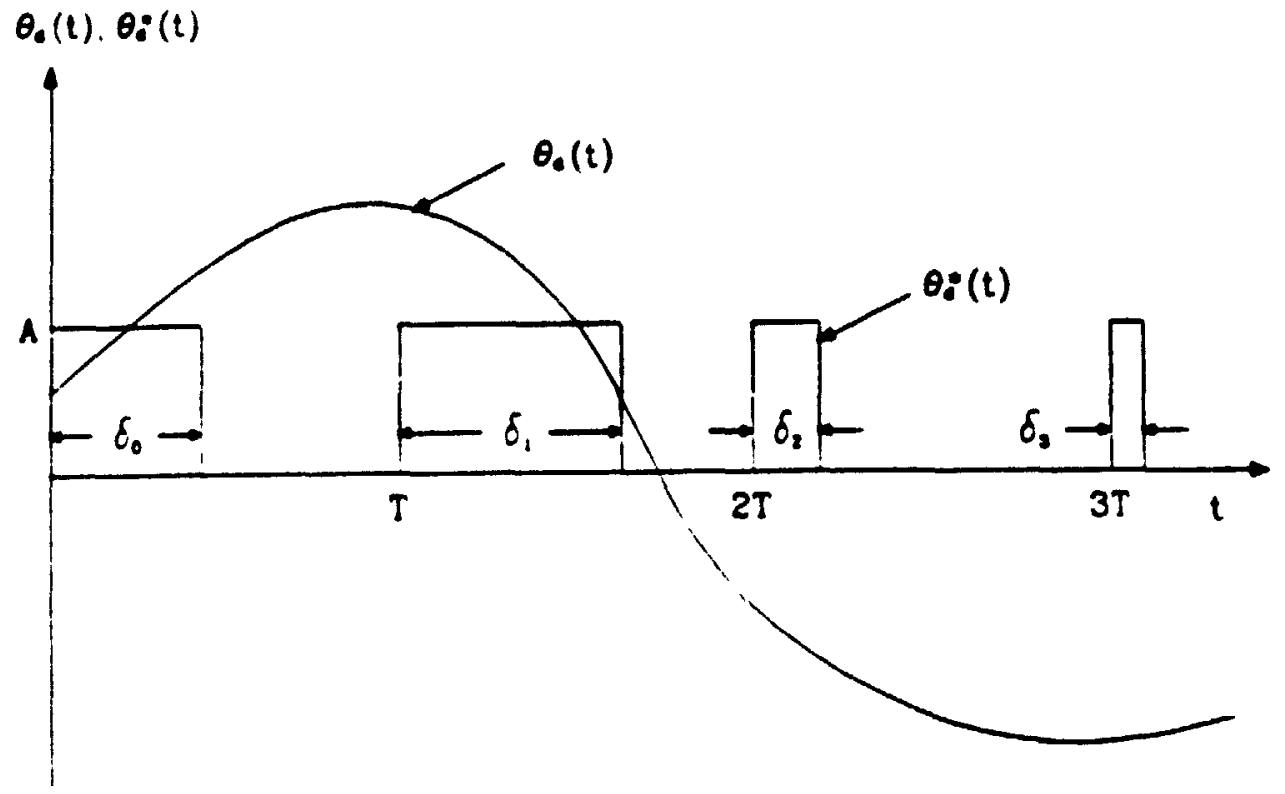

Figure 3.5: Sampling $\theta_{d}(t)$

general, we have to allow $\theta_{d}(t)$ to be either positive or negative. When $\theta_{d}(t)$ is negative, the edge from the divider ontput occurs first and the $n^{\text {th }}$ pulse can be described as

$$
\theta_{d}^{\cdot}(t)=A\left[u\left(t-n T-\delta_{n}\right)-u(t-n T)\right]
$$

Once again, the width of the pulse is given by the phase difference at the first edge

$$
\delta_{n}=k \theta_{d}\left(n T-\delta_{n}\right)
$$

and Equation 3.13 becomes

$$
\theta_{d}^{*}(t)=A\left[u\left(t-n T-k \theta_{d}\left(\delta_{n}\right)\right)-u(t-n T)\right]
$$


Equations 3.12 and 3.15 can not be combined, except possibly by including some non-linear terms to account for the variation in the position of the leading edgess of the pulses. Since this approach is beyond the scope of this thesis, we have chosen to assume that the output of the phase detector can be described by Equation 3.10, regardless of the polarity of $\theta_{d}(t)$. We feel that this assumption is valid because. for this analysis, we are interested in the general sampled-data chararteristics of the loop rather than its exact behaviour. Furthermore, this assumption corresponds to a typical application where the widths of the output pulses are small compared to the reference period. a consequence of the reference frequency being at least an order of magnitude larger than the loop bandwidth. Then, the effect of the variation in the positions of the pulses is not expected to be significant and the conclusions draw'I from this analysis should be valid for a typical application.

Based on Equation 3.12, we can describe the output of the phase detector, in general, as

$$
\theta_{d^{*}}(t)=A \sum_{n=0}^{\infty}\left[u(t-n T)-u\left(t-n T-k \theta_{d}\left(n T^{\prime}\right)\right)\right]
$$

The Laplace transform of Equation 3.16 is

$$
\begin{aligned}
\Theta_{d}^{*}(s) & =\frac{A}{s} \sum_{n=0}^{\infty}\left[\exp ^{-s n T}-\exp ^{-s\left(n T+k \theta_{d}(n T)\right)}\right] \\
& =\frac{A}{s} \sum_{n=0}^{\infty} \exp ^{-s n T}\left[1-\exp ^{-s k \theta_{d}(n T)}\right]
\end{aligned}
$$


For small $k \theta_{d}(n T)$

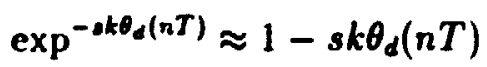

and Equation 3.18 becomes

$$
\begin{aligned}
\Theta_{d}^{*}(s) & =\frac{A}{s} \sum_{n=0}^{\infty} \exp ^{-o n T}\left[1-\left(1-s k \theta_{d}(n T)\right)\right] \\
& =\frac{A}{s} \sum_{n=0}^{\infty} \exp ^{-s n T} * s k \theta_{d}(n T) \\
& =A k \sum_{n=0}^{\infty} \theta_{d}(n t) \exp ^{-s n T}
\end{aligned}
$$

For convenience let $A=1 / k$. Then

$$
\Theta_{d}^{*}(s)=\sum_{n=0}^{\infty} \theta_{d}(n T) \exp ^{-s n T}
$$

Once again, we can use basic Fourier transformation relationships to rewrite Equation 3.18 as

$$
\Theta_{d}^{*}(j \omega)=\frac{1}{T} \sum_{k=-\infty}^{\infty} \theta_{d}(j \omega-j k 2 \pi / T)
$$

As expected, Equation 3.19 indicates that the spectrum of the output of the PFD consists of replicas of $\Theta_{d}(\omega)$ around all multiples of $f_{\bullet}$. Note, however, that it shows no attenuation of these replicas at higher frequencies, similar to the $(\sin x) / \mathrm{x}$ factor of a typical sampled-data circuit. This is a consequence of our assumption that the uidths of the pulses are small. While this assumption was useful in terms of finding an expression for $\theta_{d}^{*}(\omega)$, intuitively we feel that therc must be some high-frequency 
attenuation. The exact form of this attenuation is difficult to determine but we feel that it should resemble the $(\sin x) / x$ factor described earlier. Nevertheless. the important point is that we have established the presence of the replicas of $\theta_{d}\left(\omega^{\circ}\right)$.

Before continuing, we should recall the underlying assumption of this analysis, i.e. that the widths of the phase detector output pulses are small compared to the time between successive pulses. It seems reasonable to expect that, in an application where this assumption is not valid, the sampled-data nature of the phase detector should be less evident. Fortunately, this agrees with what we know of general, sampled.data systems where, as the width of the samples increases, the attenuation due to the (sin $x) / x$ factor also increases. Similarly, we expect that an increase in the widths of the phase detector output pulses would be accompanied by a decrease in the magnitude of the replications of $\theta_{d}\left(\omega^{*}\right)$.

The transfer function from the output of the phase detector to the output of the VCO has the form of a low-pass filter and this implies that any portions of the replicas of $\Theta_{d}(\omega)$ which fall within the loop bandwidth will appear at the VCO output. III other words, those components of $\theta_{d}(\omega)$, within a range of frequencies equal to the loop bandwidth around the harmonics of the input frequency, can appear at the VCO output due to replication. Consequently, we can think of spectral replication as a means by which components of $\theta_{d}(\omega)$ located at frequencies beyond the loop 
bandwidth can still have an impact on the output. The significance of this point must be stressed since it represents a major distinction between an implementation of the loop based on a charge-pump phase detector and a continuous version employing a multiplier as the phase detector. In the latter case, components of $\theta_{d}(\omega)$ beyond the loop bandwidth have little effect on the VCO output.

One of the main concerns regarding the effect of the replication of $\theta_{d}(\omega)$ is that a component at a harmonic of the reference frequency, $f_{1}$, may be replicated to dc. thereby causing a shift in the average output frequency of the VCO. Fortunately, it turns out that this is not possible, as is evident in Figure 3.6 which shows an example of the reference signal and the divider output containing a single phase noise component at $3 f_{1}$. We can see that this phase noise has no effect on the zero-crossings of the divider output and, so, has no effect on the output of the phase detector. In the more general case where the phase noise contains a phase shift relative to the reference signal the result is, initially, a shift of the zero-crossings of Div compared to Ref and a dc component at the output of the phase detector. However, as is generally the case, the feed' ck loop acts to remove this component and, under steady-state conditions, the zero-crossings of Ref and Div are once again identical.

The simulation structure described in this thesis can be used to determine the level of phase noise around the average output frequency provided the source of the phase 


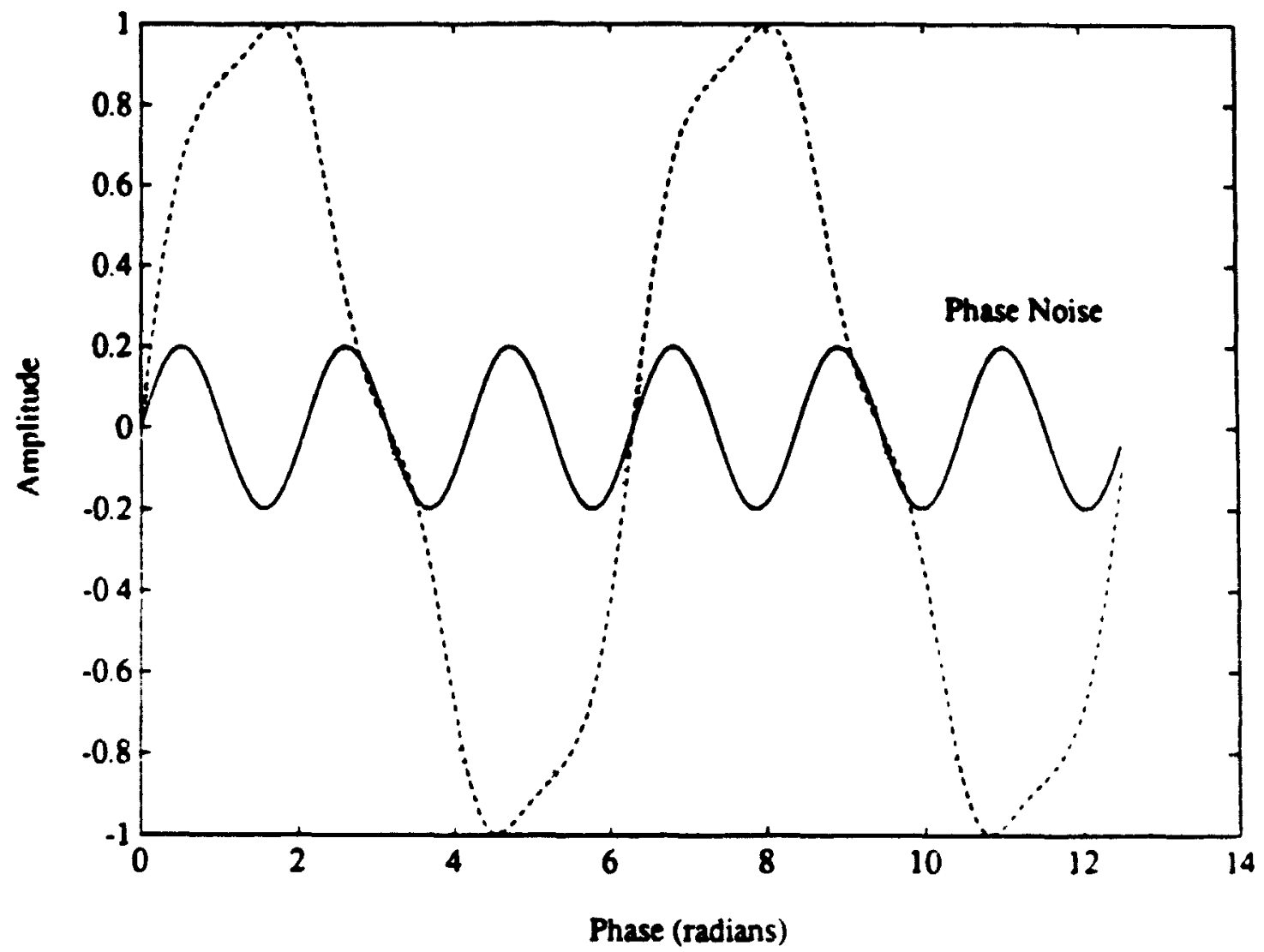

Figure 3.6: Ref $\ldots$, Div $\cdots$, and $\theta_{d}(t) \longrightarrow$ when $B=0.2$ and $\omega_{\theta_{n}}=3.0 * \omega$, difference between Ref and Div can be characterized in the time-domain. Recall that, in an ideal loop, Ref and Div are identical. Typically, we might be interested in the contribution to $\theta_{d}(\omega)$ by VCO phase noise, coupled power supply noise, or thermal noise in the loop filter but, in such cases, a time-domain description is difficult, except by statisitcal means. Fortunately, this is not a problem for the other aspect of a monolithic implementation of the loop of interest here, namely the non-linear 
transfer function of sypical integrated charge-pump phase detectors. We will see that a representation of the common sources of the non-linearity, suitable for inclusion within the simulation model of the loop, is possible. The results of these simulations are given in Chapter 4 but the next sections provide some background in order that these results may be interpreted properly.

\subsection{Non-Linear Charge-Pump Phase Detector}

As an introduction to the topic of a non-linear charge-pump phase detector, consider a general non-linearity expressed as a third-order polynomial.

$$
v_{0}=k_{1} v_{1}+k_{2} v_{1}^{2}+k_{3} v_{1}^{3}
$$

When the input is a single frequency, $f_{1}$, we can see that the output consists of components at this frequency and at its second and third harmonic. However, when the input includes a second frequency, $f_{2}$, the output contains components at $f_{1}, f_{2}, 2 f_{1}, 2 f_{2}, 3 f_{1}$, and $3 f_{2}$ and intermodulation products at $f_{1} \pm f_{2}, 2 f_{1} \pm f_{2}$ and $f_{1} \pm 2 f_{2}$. In general, we expect that, for an arbitrary input spectrum, a non-linear device will lead to an out put with a spectrum distributed over a much wider bandwidth. However, it is also possible that some of the intermodulation products may fall near dc, as in the case of the components at $f_{1}-{ }_{2}{ }_{2}, 2 f_{1}-f_{2}$, and $f_{1}-2 f_{2}$ in the above example. In a frequency 
synthesizer application, these components may fall within the loop bandwidth, once again leading to an increase in the level of phase noise around the average output frequency. As a result, one of the aims of this thesis is to determine if any of the common sources of a non-linearity in a charge-pump phase detector is capable of this type of behaviour.

Also of interest is the possibility that the wideband components generated by the non-linearity may be replicated within the loop bandwidth. In Chapter 1 we saw that there is some question of whether the sampling by the phase detector commes befose the non-linearity or after. This question can be resolved by considering the common causes of the non-linearity: unequal propagation delays in the digital logic of the phase/frequency detector and an asymmetry between the current sources of the charge-pump. In both cases we find the most useful way to represent the nonlinearity is in terms of its effect on $Q_{n}$. the amount of charge transferred to the lowp filter. Since $Q_{n}$ is a function of $\Delta t_{n}$, the time between the $n^{\text {th }}$ edges of the reference and feedback signals, we can think of it as a representation of the $n^{\text {th }}$ sample of $\theta_{d}(\ell)$. Then, if either non-linearity causes a distortion in the amount of charge transferred, it has, in effect, distorted the value of this sample. In other words, the non-linearity is part of the sampling action. A similar situation can arise if a simple sample-andhold circuit contains a non-linearity; consider a case where a third-order polynomial 
causes a distortior vi the samples of a sinusoidal input with a frequency $f_{i}$. The key point is that if the sampling frequency, $f_{0}$, is identical to $3 f_{1}$, the output will contain a component at $\mathrm{dc}$; in effect, the non-linearity generates the third harmonic of $f_{i}$ at $f_{0}$ which is aliased to dc. Then it is appropriate to think of the non-linearity as acting on the continuous input with the result being sampled. Note that if we chose to view the non-linearity as following the sampling, we wo ild be unable to explain the appearance of the component at dc. In a similar manner, we believe that a nonlinearity in a charge-pump phase detector can be viewed as distorting $\theta_{d}(t)$ with the phase detector sampling the result. Consequently, the components due to the nonlinearity can be replicated within the loop bandwidth, leading to an increase in phase noise at the VCO output; whether or not this occurs depends on the characteristics of the individual non-linearities, described in the next sections.

\subsubsection{Asymmetrical Propagation Delays}

The schematic of the phase/frequency detector of Figure 3.7 shows that the propagation delays from the inputs to each output are unequal. A Ref edge will cause the Il output to become active after two gate delays while a Div edge causes $U$ to be reset after propagating through three NAND gates and a flip-flop. The effect of this 
asymmetry can be determined with the aid of Figure 3.8 which shows the current into the loop filter as a function of time for both positive and negative phase errors. The finite rise and fall times are intended to reflect the non-ideal nature of the $V$ and D signals and also of the transmission gates used to connect the charge-pump to the loop filter. $Q_{n}$, the total charge transferred through the loop filter f or a given time difference, $\Delta t_{n}$, between edges of Ref and Div is the area under the current curve and can be represented by

$$
Q_{n}=I_{p} \Delta t_{n}-Q_{A}+Q_{B}
$$

where $I_{p}$ is the value of the charge-pump current sources and $Q_{A}$ and $Q_{B}$ are the areas of the regions shown in Figure 3.8. If $Q_{A}=Q_{B}$ then $Q_{n}\left(\Delta t_{n}\right)$ is a linear function passing through the origin. However, if $Q_{A}<Q_{B}$ then $Q_{n}\left(\Delta t_{n}\right)$ is linear except for small $\Delta t$ where the rising and falling transients meet; since the falling transient lasts longer than the rising, more charge is transferred through to the feedback elements of the loop filter than was necessary. Similarly, when $Q_{A}>Q_{B}$, the rising transient is longer and less charge is transferred than required. The three cases are shown in Figure 3.9.

Measurements of physical devices indicate that it is the $Q_{A}>Q_{B}$ case which occurs most often [8]. Then, by our earlier argument, we can model the non-linear 


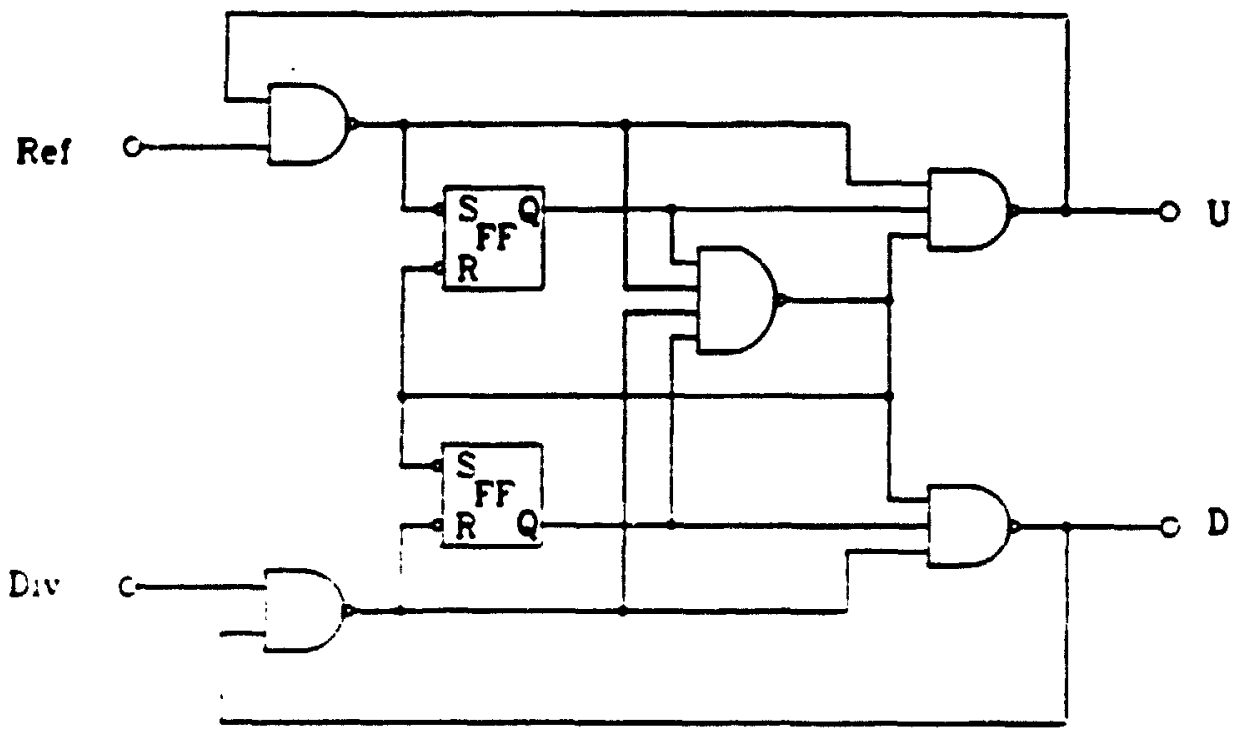

Figure 3.7: Phase-Frequency Detector

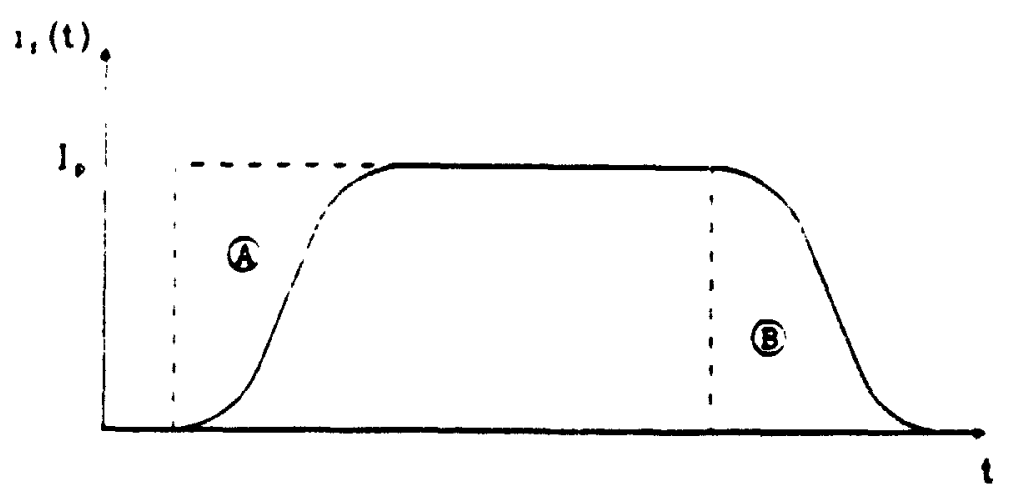

Figure 3.8: Current into Loop Filter 


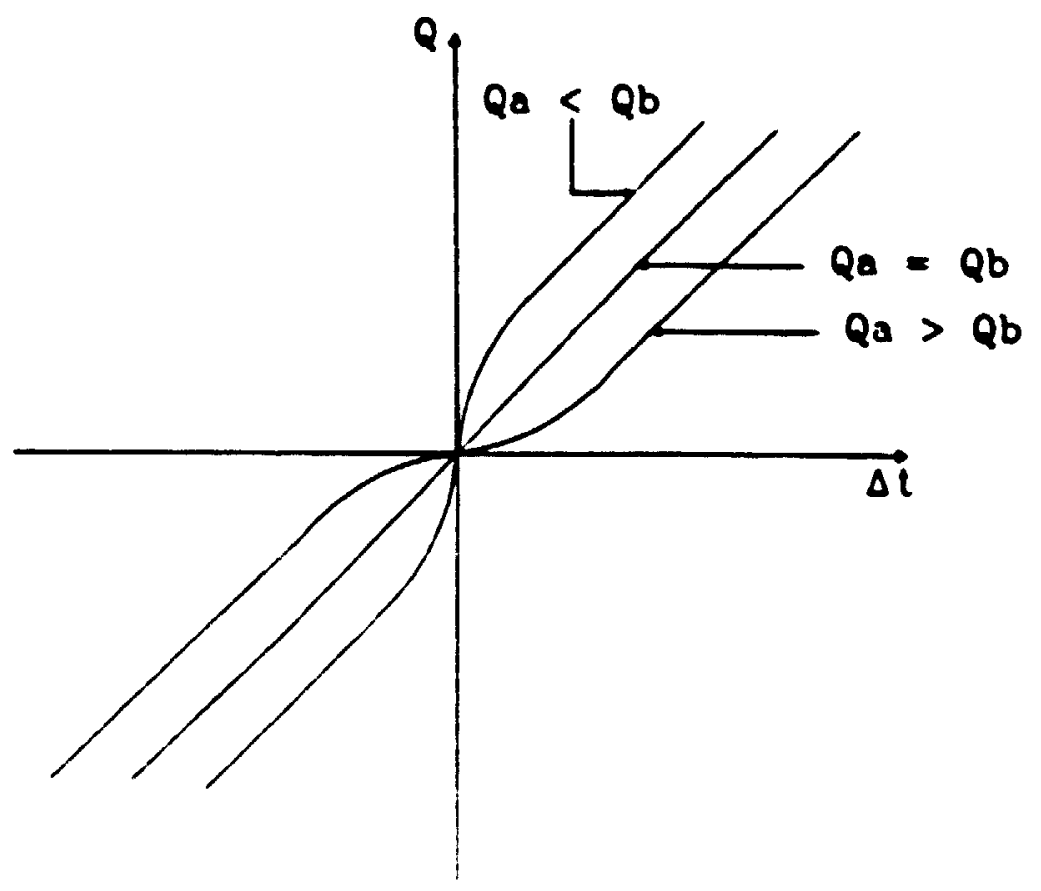

Figure 3.9: $Q_{n}$ versus $\Delta t_{n}$ for Asymmetrical Propagation Delays

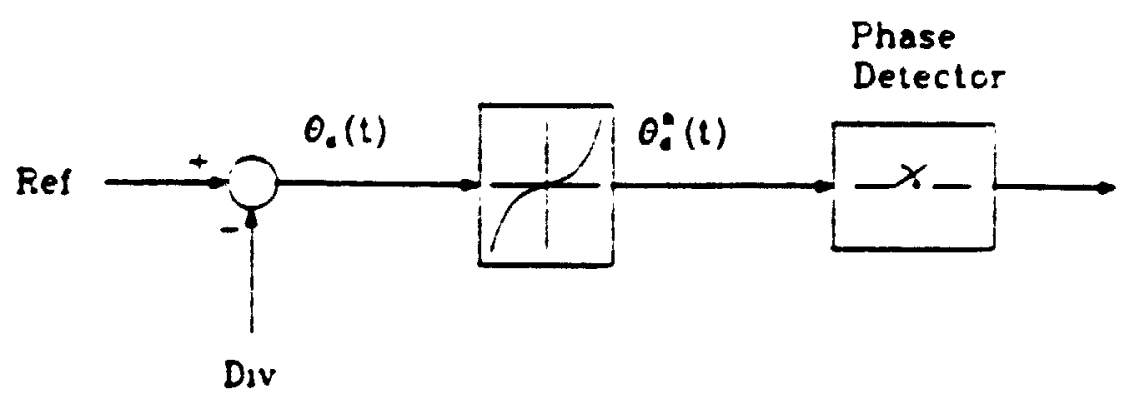

Figure 3.10: Modelling a Charge-Pump Phase Detector with Asymmetrical Propagation Delays 
phase detector in terms of a non-linearity, $\mathrm{f}(\mathrm{t})$, shown in Figure 3.10, applied to $\theta_{d}(t)$ with the result, $\theta_{d}^{\text {nl }}(t)$, sampled by a linear phase detector. For small $\theta_{d}(t), f(t)$ has the form of a third-order polynomial; consequently, we expect $\Theta_{j}^{\prime \prime}(\omega)$ to be distributed over a bandwidth three times greater than that of $\theta_{d}(\omega)$, thereby increasing the probability of replication of spectral components within the loop bandwidth. As well, a non-linearity of this type is capable of generating spurious components through intermodulation of $\theta_{d}(\omega)$, as described earlier. In the next chapter we will attempt to develop some quantitative idea of the effect of the non-linearity on the loop output by incorporating it within our simulation structure.

\subsubsection{Asymmetrical Current Sources}

If the two current sources of the charge-pump are unequal, the relationship between $Q_{n}$ and $\Delta t_{n}$ will resemble that shown in Figure 3.11. The value of the current source supplying positive charge to the loop filter, $I_{p p}$, represents the slope of the curve for positive $\Delta t_{n}$ while $I_{p m}$, the value of the current source drawing charge from the loop filter, corresponds to the slope of the curve for negative $\Delta t_{n}$. Once again, it is possible to view the non-linearity as acting on $\theta_{\dot{c}}(t)$ with the phase detector sampling the result but, in this case, it is not easy to determine the nature of the interaction 


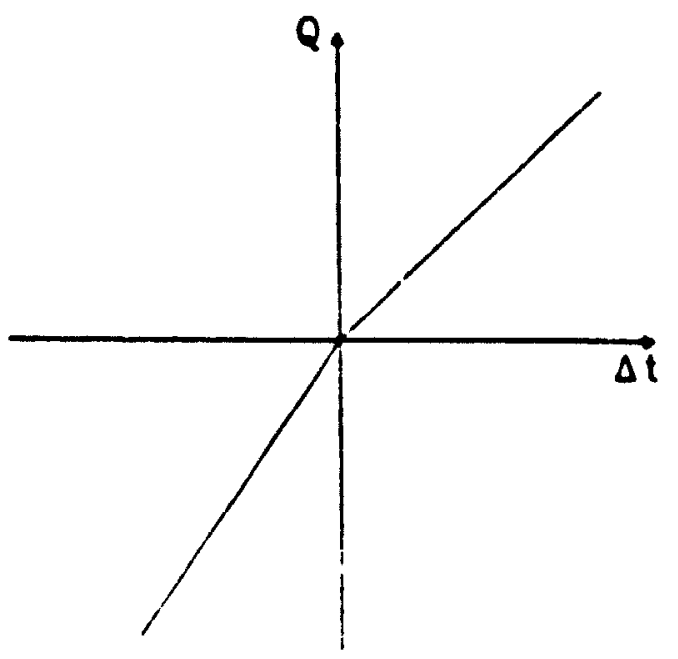

Figure 3.11: $Q_{n}$ versus $\Delta t_{n}$ for Asymmetrical Current Sources

between the sampling and the non-linearity in an analytical fashion. However, we can say that, since the relationship between $Q_{n}$ and $\Delta t_{n}$ is not an odd function, a power series representation will contain both even and odd-order terms. As a result, it seems that an asymmetry between sources can also lead to an increase in the level of phase noise in the region of noise-shaping around the average output frequency, either through intermodulation or through sampling and replication. Fortunately, this type of non-linear behaviour can be incorporated very simply within the simulation model of the loop and we should be able to develop some quantitative idea of its effect on the output of the VCO. 

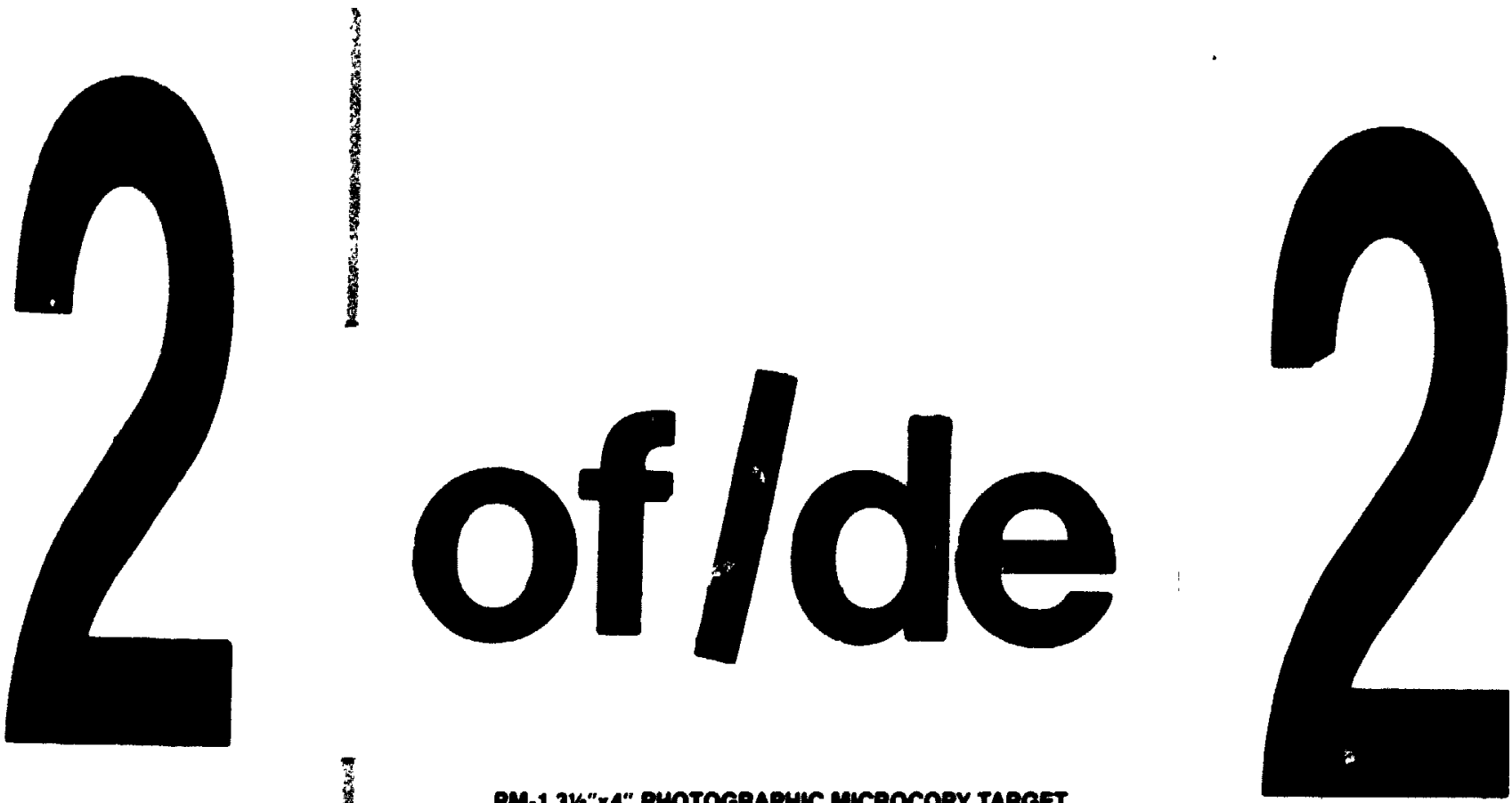

PM-1 3\%" "x4" PHOTOGRAPHC MICROCOPY TARGET NeS 1010a ANEARSO H2 EQUNALENT

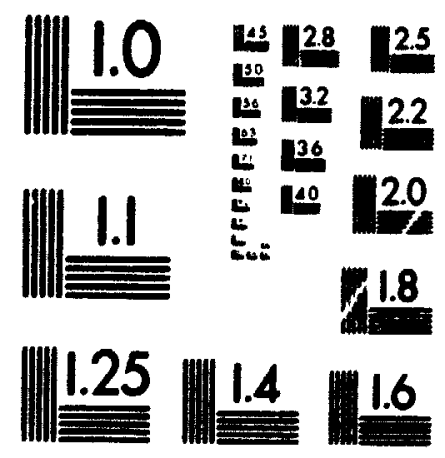

PRECISIONM RESOLUTION TARGETS

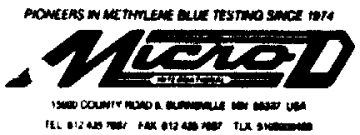




\subsection{Summary}

In this chapter, we have identified potential sources of an increase in phase noise in the region of noise-shaping around the average output frequency of a fractional$\mathrm{N}$ frequency synthesizer when a charge-pump pliase detector is used instead of a multiplier. Specifically, we have determined that the charge-pump phase detector can generate replicas of the spectrum of the phase difference between Ref and Div and that portions of these replicas can appear near dc. As well, a non-linear phase detector transfer function can generate low-frequency phase noise components either through replication of the high-frequency components due to the non-linearity or through intermodulation of the spectrum of the phase difference. In the next chapter, we will attempt to develop some idea of the magnitude of the increase in phase noise at the output. 


\section{Chapter 4}

\section{Simulation Results}

\subsection{Introduction}

The purpose of this chapter is to examine the time and frequency-domain behaviour of a fractional- $\mathrm{N}$ frequency synthesizer using a customized simulation model. In addition to establishing the general characteristics of the loop, we are particularly interested in the elements of its behaviour related to the use of a charge-pump phase detector. Earlier in this thesis, we concluded that the sampled-data nature of this type of phase detector implies that components of $\Theta_{d}(\omega)$ beyond the loop bandwidth may be replicated within the region of noise-shaping around the average output frequency. As well, we saw that a typical, non-linear charge-pump phase detector can contribute 
to this phase noise through intermodulation of $\theta_{d}(\omega)$ or through replication of the spectral components generated by the non-linearity. Both the sampled-data and non-linear characteristics of the phase detector represent means by which spectral components beyond the loop bandwidth can still have an impact on the VCO output. Ideally, the simulation results presented here would be able to demonstrate that these conclusions are valid. Failing this, we should at least be able to develop some idea of the relationship between an important element of a monolithic implementation of the loop, a charge-pump phase detector, and the overall response of the system.

This chapter is divided into three sections: the first deals with the basic behaviour of the loop and includes a review of our approach to its simulation and a description of the loop under investigation. We present examples of the time and frequency-domain behaviour of the system and explain how these results are to be interpreted. The second section focuses on the sampled-data characteristics of the loop. We will attempt to show that when the reference signal contains an secondary component representing phase noise, this component appears at the VCO output at other frequencies in addition to the original. We will use this fact as proof that the spectrum of the phase difference between the reference and feedback signals is being replicated. Note that the phase noise is included in the reference signal here for convenience but in a typical application the main components of $\theta_{d}(\omega)$ are more likely to be part of the 
feedback signal from, for example, power supply noise in the dual-modulus divider. In the third section of this chapter, we will consider the non-linear characteristics of the phase detector and loop. Finally, throughout this chapter we plan to demonstrate the versatility of the simulation model and identify the main areas of investigation where it can be applied.

\subsection{Basic Loop Behaviour}

Recall that the approach to simulation of the frequency synthesizer taken in this thesis is based on an iterative solution of the time between corresponding edges of the reference and feedback signals, Ref and Div. In other words, the $n^{\text {th }}$ value of $\Delta t$ is found by first generating a new bit from the sigma-delta modulator, setting the division ratio, $N$, appropriately, and then calculating the time, $t_{0}$, required for the phase of the VCO output to change by $2 N \pi$ radians. Then the value of $\Delta t[m]$ is given by

$$
\Delta t[m]=\Delta t[m-1]+t_{0}-T
$$

and, with this value, we can calculate the instantaneous VCO output frequency throughout the $n-1^{\text {th }}$ interval. The simulation structure used to carry out this calculation was written in $\mathrm{C}$ and is based on a description of the time-domain be- 
haviour of the loop. The program listing is included in Appendix A for reference.

The loop used in all simulations described in this chapter is shown in Figure 4.1 and is based on a typical application where frequencies in the range $50 \mathrm{MHz} \cdot 52.5 \mathrm{MHz}$ are to be synthesized from a $2.5 \mathrm{MHz}$ reference signal. This implies that we use a 20/21 divider in the feedback loop. As well, the damping factor, $\zeta$, was chosen as 0.7 and the nominal unity-gain bandwidth as $\omega_{i} / 100$. Note that these values are easily changed. Also note that, for simplicity, a reference frequency of $250 \mathrm{~Hz}$, rather than 2.5 $\mathrm{MHz}$, was used in the simulations. When the divider is controlled by the secondorder sigma-delta modulator of Figure 4.2, the desired output frequency is specified in terms of an integer, $P$, between $-10,000$ and $+10,000$. For example, choosing a value of zero gives the nominal output frequency of $5125 \mathrm{~Hz}$ while a value of 1140 produces an average frequency of

$$
\begin{aligned}
\omega_{a v} & =250 *\left(20.5+\frac{1140}{20000}\right) \\
& =5139.25 \mathrm{~Hz}
\end{aligned}
$$

Figure 4.3 shows a result from our simulation model of how the instantaneous VCO frequency changes with time when the average output frequency is $5139.25 \mathrm{~Hz}$. We can see that the sharp transitions in the VCO frequency correspond to the charge- 


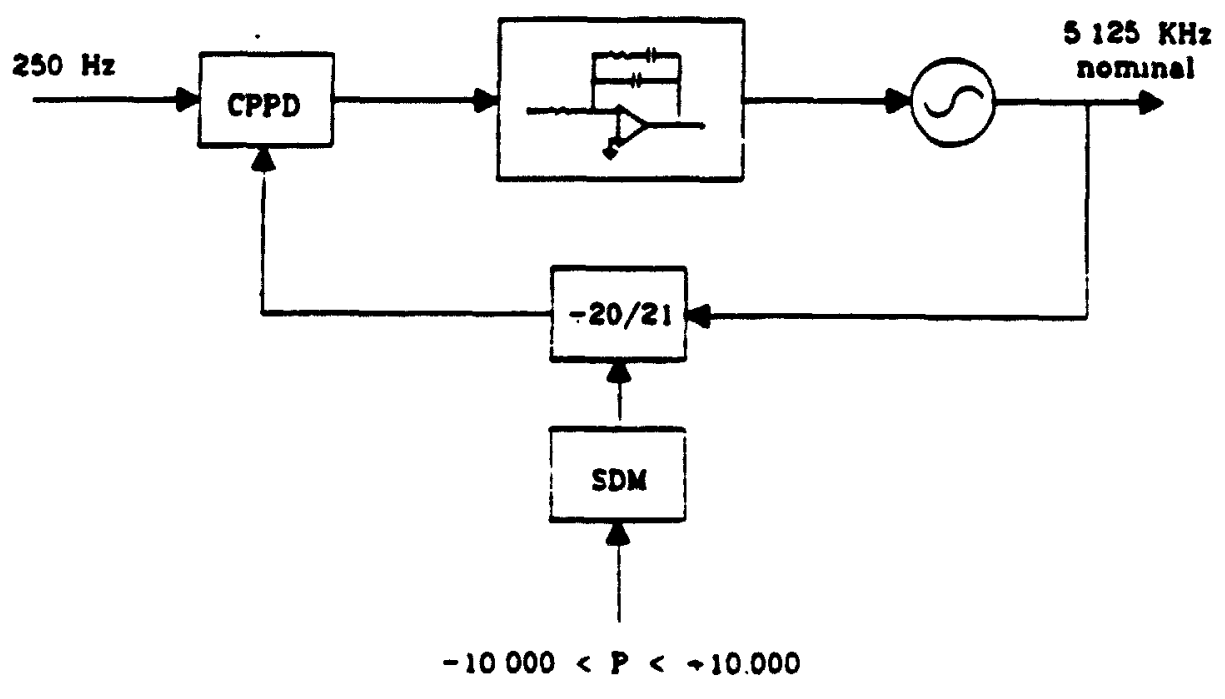

Figure 4.1: Fractional-N Frequency Synthesizer as Simulated (Frequencies are scaled by a factor of 10,000 from a typical application)

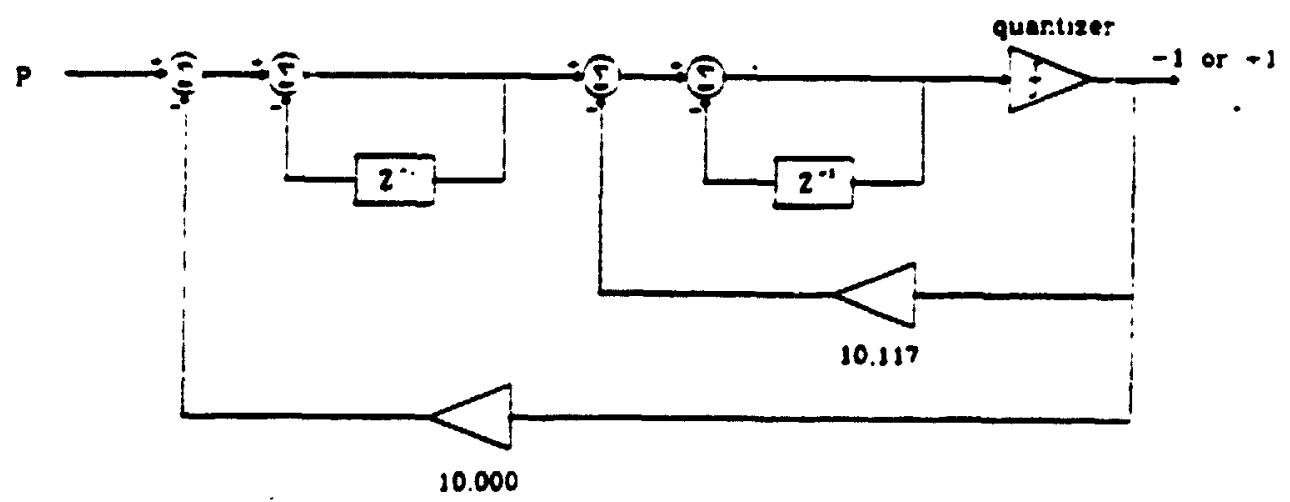

Figure 4.2: Second-Order SDM used in Simulations $(-10,000 \leq P \leq 10,000)$ 
pump being turned cn; when the charge-pump is turned off, the output frequency decays towards its average value. We can determine the effect of these transitions on the stability of the output frequency by performing a Discrete Fourier Transform on the samples of the frequency to give $S_{\omega \omega_{0}}(\omega)$. Shown in Figure 4.4 is the result of a 65,536-point transform, performed on one frame of data with rectangular windowing, corresponding to roughly 300 cycles of the reference input. We should note that, since the DFT is carried out on samples of the instantaneous output frequency, the form of the spectrum shown in Figure 4.4 is different than what we would see with a spectrum analyzer. In Chapter 2 we determined that, in this transformation from the time to the frequency domain, the average output frequency is represented as the magnitude of a component at dc. As well, we saw that the spectrum of the phase noise evident in Figure 4.4 is identical to what we would see with a spectrum analyzer except that it is centered at $\mathrm{dc}$ and is scaled by $20 \mathrm{db} / \mathrm{dec}$. As a result, the phase noise, in effect, becomes frequency noise. Despite these differences, this type of frequency-domain representation of the behaviour of the loop is useful in determinirg the relationship between the particular aspects of the implementation of the loop and the spectral purity of the VCO output.

The shaping of the divider noise, corresponding to the use of the second-order sigma-delta modulator of Figure 4.2 as the divider controller, is clearly evident in 


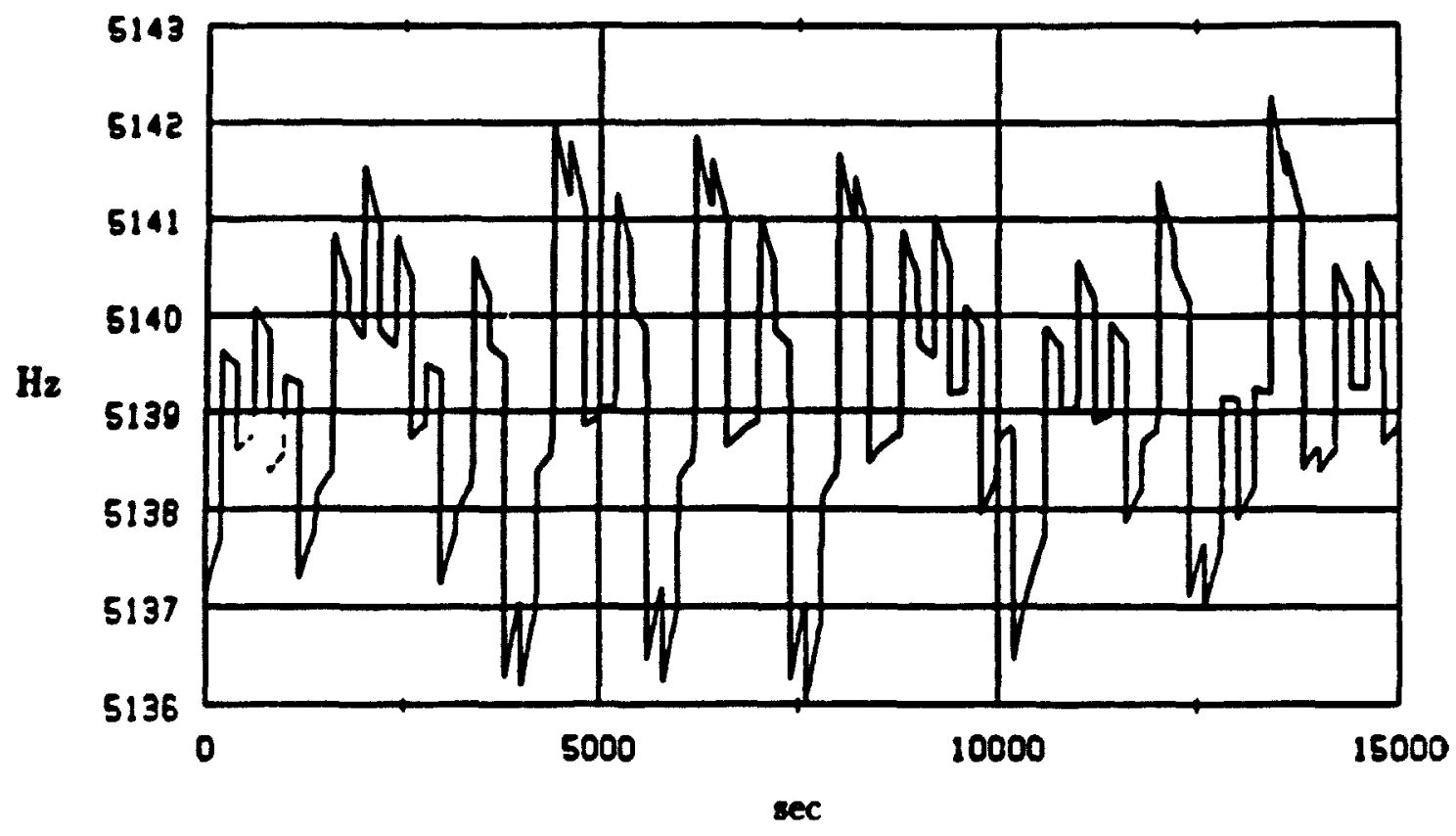

Figure 1.3: $\omega_{\text {ino }}(t)$ when $P=1140$ (note: total duration is $0.3 \mathrm{sec}$ )

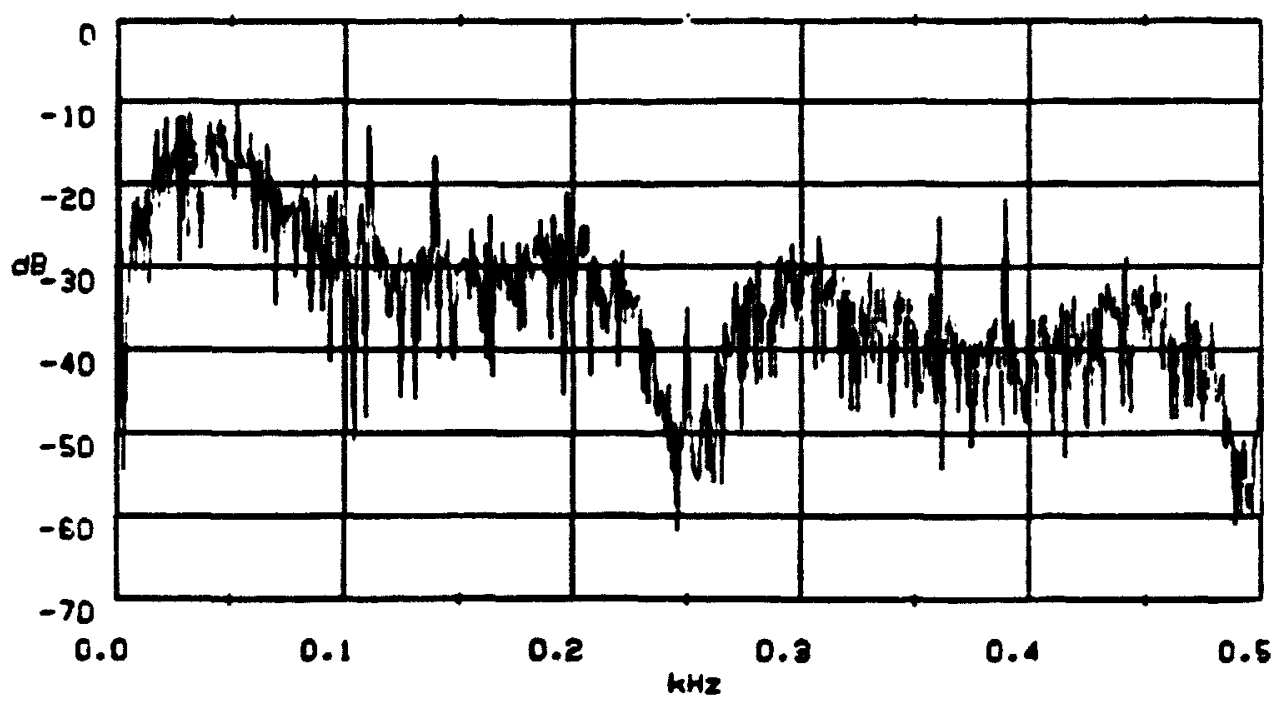

Figure 4.4: $S_{w_{\text {wao }}}(\omega)$ when $P=1140$ 


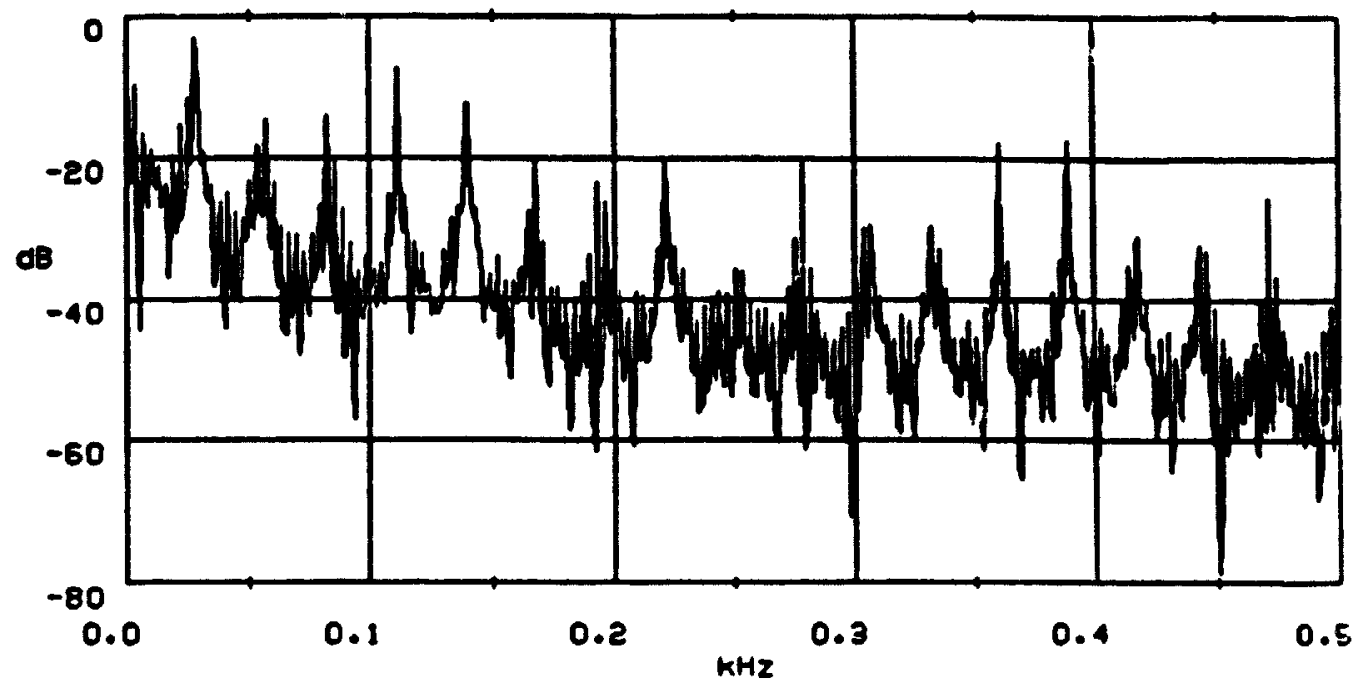

Figure 4.5: $S_{\omega_{\mathrm{m} n} \text { o }}(\omega)$ when $\mathrm{P}=1140$ (Divider Controlled by a Digital Phase Accumulator)

Figure 4.4 as the region of low phase noise near dc. Note that the vertical scale has been expanded to give more detail to the phase noise. As a result, the component at $\mathrm{dc}$, sepresenting the average output frequency, is not shown. In comparison, $S_{\omega_{\text {moo }}}(\omega)$ for the identical loop under the control of a digital phase accumulator is shown in Figure 4.5; here, the divider noise exhibits a much stronger periodicity with a larger portion of $N_{d}(\omega)$ occurring near the average output frequency. Intuitively, we expect that this will tend to obscure the frequency of the output.

Figure 4.4 also shows the effects of the presence of the charge-pump phase detector. In Chapter 1, we noted that the divider noise spectrum, $N_{d}(\omega)$, and therefore $S_{\omega_{\text {m.n }}}$ 
are expected to contain nulls at all harmonics of the reference frequency, $250 \mathrm{~Hz}$, but this is clearly not the case. We believe that the reason for the presence of the components at $250 \mathrm{~Hz},: 00 \mathrm{~Hz}$, etc., is the fact that the output of the loop filter goes through a transient on every cycle of the reference signal, regardless of whether the division ratio is changed. In other words, these components are not part of $N_{d}(\omega)$ but are a consequence of the charge-pump phase detector. Furthermore, the transients in the loop filter output can, in fact, take on either of two polarities and this suggests that the output spectrum shovid contain components at 'rational harmonics' of the reference frequency, i.e. $125 \mathrm{~Hz}, 375 \mathrm{~Hz}$, etc.. For example, if the phase difference between the input and feedback signals continuously alternates between positive and negative polarities, the fundamental of the transient signal applied to the VCO is actually at $125 \mathrm{~Hz}$. The fact that, instead of a single component at these rational harmonics, Figure 4.4 shows a component just above and below these frequencies is possibly the result of there being an inequality between the time required for a division by 20 and that for a division by 21 . A $i$ any rate, these components are not expected to have a significant effect on the output unless the magnitude of the transients in the output frequency become comparable to the average value. Nevertheless, they do demonstrate what sort of an effect the charge-pump phase detector can have on the output of the VCO. 


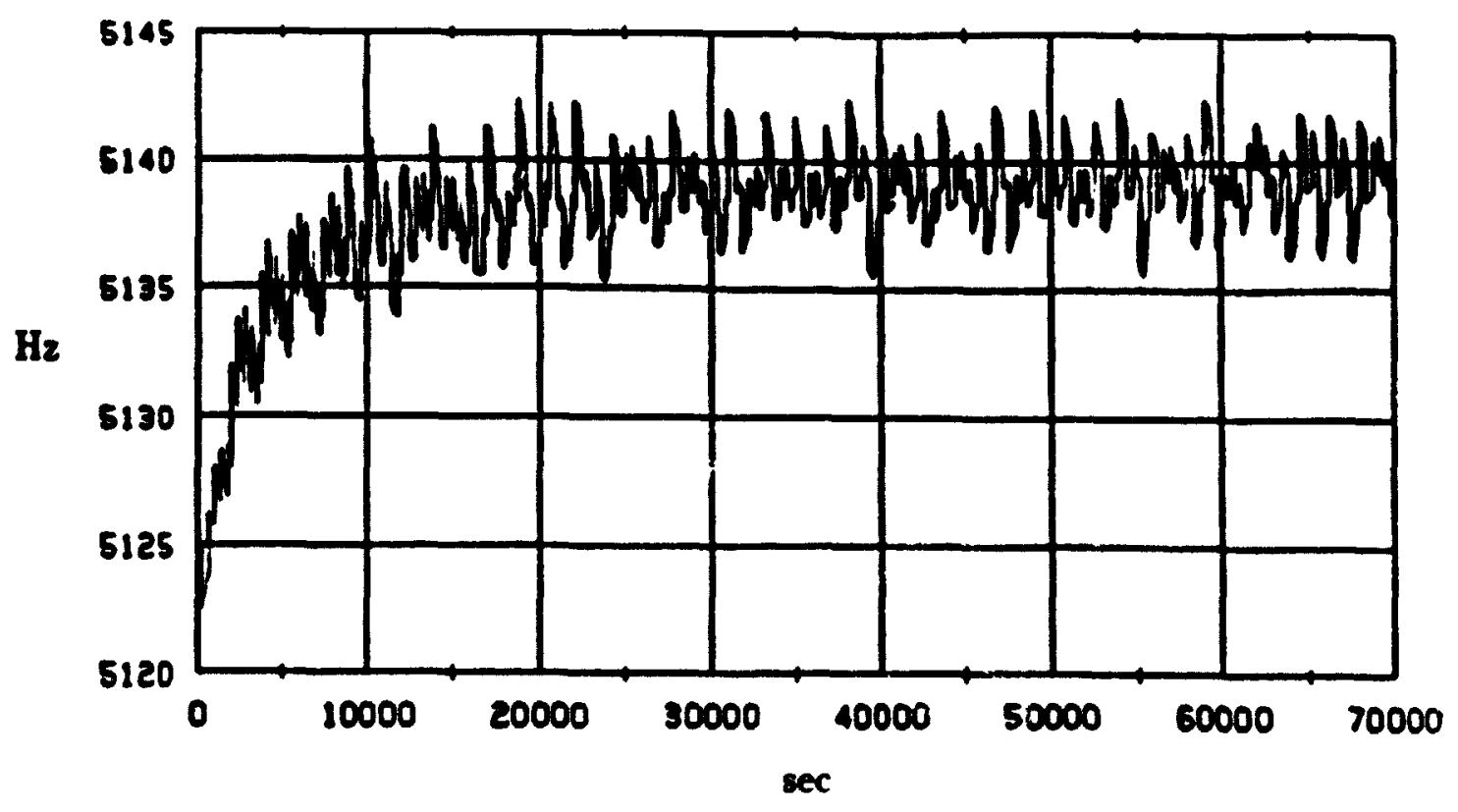

Figure 4.6: Transient Loop Behaviour - $\omega_{\text {ino }}(t)$

Before turning to the sampled-data characteristica of the loop, we will examine some of the other areas in which the simulation model developed in this thesis can be applied. First, consider the transient response of the loop. Shown in Figure 4.6 is the instantaneous output frequency as the loop changes from an initially unstressed state, i.e. $P=0$, to one corresponding to a value of $P=1140$. Intuitively, we expect that the discrete-time nature of the charge-pump phase detector will tend to slow the overall transient response of the system but with the aid of the simulation model we can put this belief in more quantitative terms.

As well, we can determine the effect of a change of one of the loop parameters such as the unity-gain bandwidth, ws. Shown in Figure 4.7 are the steady-state time- 

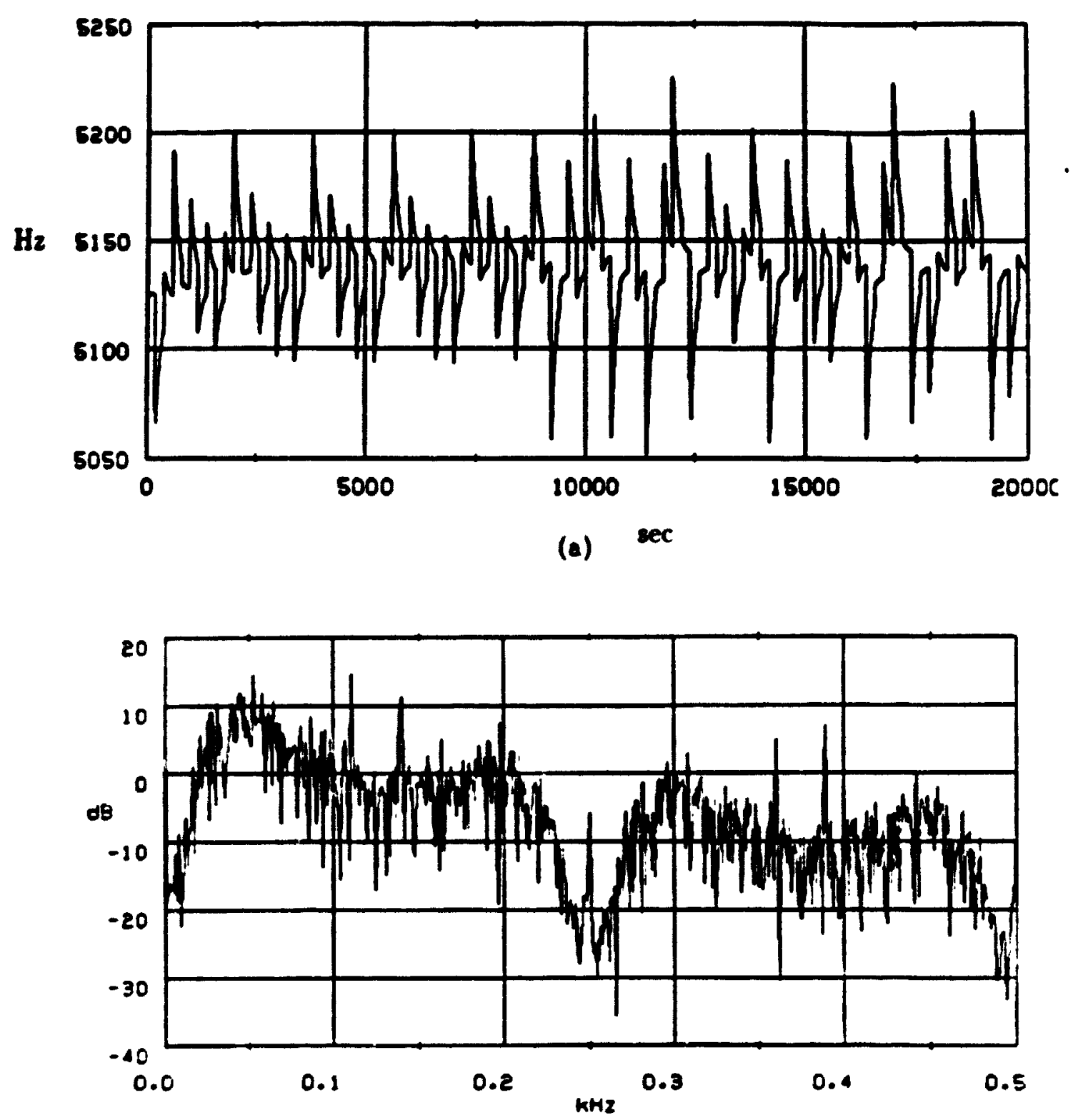

(b)

Figure 4.7: $\omega_{\text {ino }}(t)$ and $S_{\omega_{0.0}}(\omega)$ when $\omega_{b}=\omega_{i} / 20$ 
domain and frequency-domain responses of the loop when $\omega_{b}$ is increased from $\omega_{i} j 100$ to $w_{i} / 20$. As expected, the wider bandwidth causes an increase in the magnilude of the time-domain transients associated with a change in the divider count. The result is a significant increase in the level of unwanted signals at the output. While the widths of the regions of shaped noise around $0 \mathrm{~Hz}, 25 \cap \mathrm{Hz}$, and $500 \mathrm{~Hz}$ have increased, the depths of these regions have decreased, i.e. the overall level of noise has increased by about $20 \mathrm{db}$. However, the increased width of the low noise regions suggests that there might be some advantage to increasing the loop bandwidth. In ceriain circumstances, it may be that the increased level of phase noise in these regions, corresponding to the wider bandwidth, is more than offset by t..e fact the the spectral shaping is present over a wider portion of the spectrum, with the result being a decrease in the total noise power near the average output frequency and its harmonics. Investigation of this question requires more extensive simulations.

\subsection{Sampled-Data Characteristics}

Being able to accurately simulate the behaviour of the loop is particularly useful in attempting to verify its sampled-data characteristics. In Chapter 3 we concluded that, because a charge-pump phase detector responds to discrete events at its inputs, 
it is, in effect, a sampled data element and the result is replication of the spectrum of the phase difference and an increase in phase noise at the VCO output over that of a continuous loop. We can test this conclusion by including sinusoidal phase noise within the reference signal

$$
\operatorname{Ref}=A \sin \left(\omega_{i} t+B \sin \left(\omega_{\theta_{n}} t\right)\right)
$$

Since the additional signal strictly represents phase noise, it will contribute a single component, at $\omega_{\theta_{n}}$, to $\Theta_{d}(\omega)$. Then, if $\Theta_{d}(\omega)$ contains a component at a particular frequency, we expect the spectrum of the instantaneous output frequency to contain a component at the same frequency, but scaled to account for the transformation from phase to frequency. In other words, the sinusoidal phase noise should contribute a component at $\omega_{\theta_{n}}$ to the spectrum of the output frequency.

Figure 4.8 shows the results of the simulation in the frequency domain when $\omega_{\theta_{n}}=0.3 * \omega_{i}$, i.e. $75 \mathrm{~Hz}, \mathrm{~B}=0.3$, and the loop bandwidth is $\omega_{i} / 20$. The important point to note is that, in addition to the expected component at $\omega_{\theta_{n}}$, there are other components located at $\omega_{i}-\omega_{\theta_{n}}, \omega_{i}+\omega_{\theta_{n}}, 2 * \omega_{i}-\omega_{\theta_{n}}$, etc.. and we can conclude that these components are due to the replication of $\theta_{d}(\omega)$.

The significance of this conclusion is demonstrated by the simulation results shown in Figure 4.9. In this case, the frequency of the sinusoidal phase noise has been 


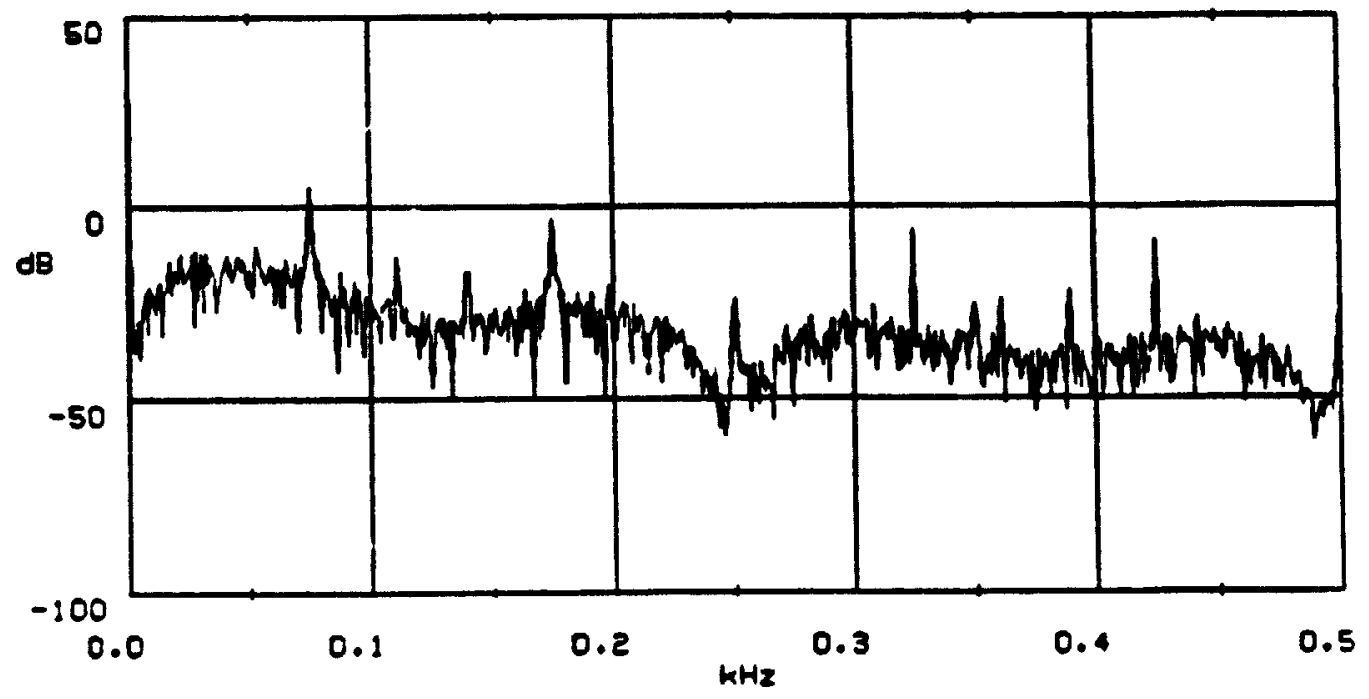

Figure 4.8: $S_{\omega_{1} \text { o }}(\omega)$ when $\omega_{0}=0.3 * \omega_{i}$

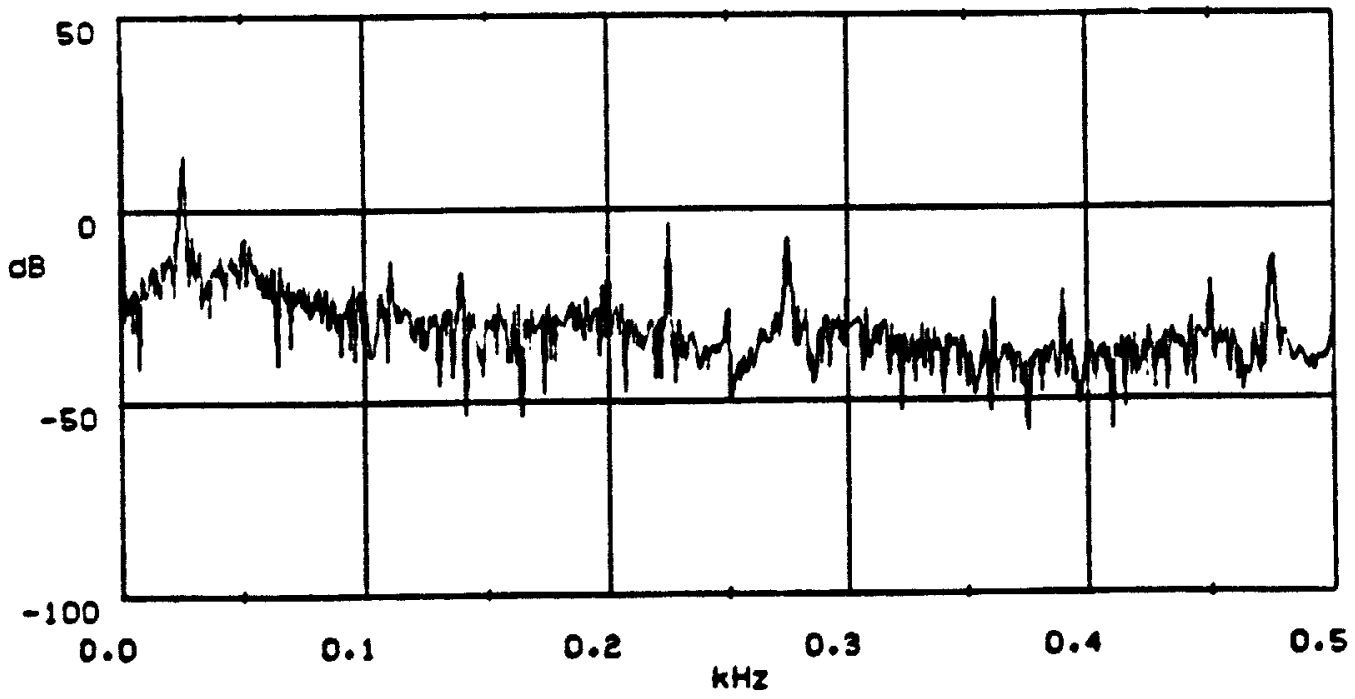

Figure 4.9: $S_{\omega_{\text {ino }}}(\omega)$ when $\omega_{0_{n}}=1.1 * \omega_{i}$ 
increased to $1.1 * \omega_{i}$. A classical frequency synthesizer would show little response to a component at this frequency since it falls so far above the loop bandwidth of $\omega_{i} / 20$. However, we can see that, because of the replication of $\theta_{d}(\omega)$, a spurious component occurs near the main component at dc, the magnitude of which represents the average output frequency. In a typical loop, such components of $\theta_{d}(\omega)$ will tend to degrade the shaping of the divider noise and obscure the desired output frequency. In a frequency synthesizer application this must be considered as significant.

\subsection{Non-Linear Phase Detector}

Now that we developed a better idea of the sampled-data nature of a charge-pump phase detector, we can turn our attention to the effect of a non-linear phase detector transfer function. The important question is whether or not the typical sources of a non-linear transfer function are capable of generating spurious components within the region of noise-shaping around the average output frequency, either through acting directly on the phase difference or through spectral replication.

Consider a phase detector with a non-linear transfer function due to an asymmetry between propagation delays in the phase/frequency detector. Recall that $\Delta t_{n}$, the time between the $n^{\text {th }}$ edges of Ref and Div, and $Q_{n}$, the amount of charge transferred 


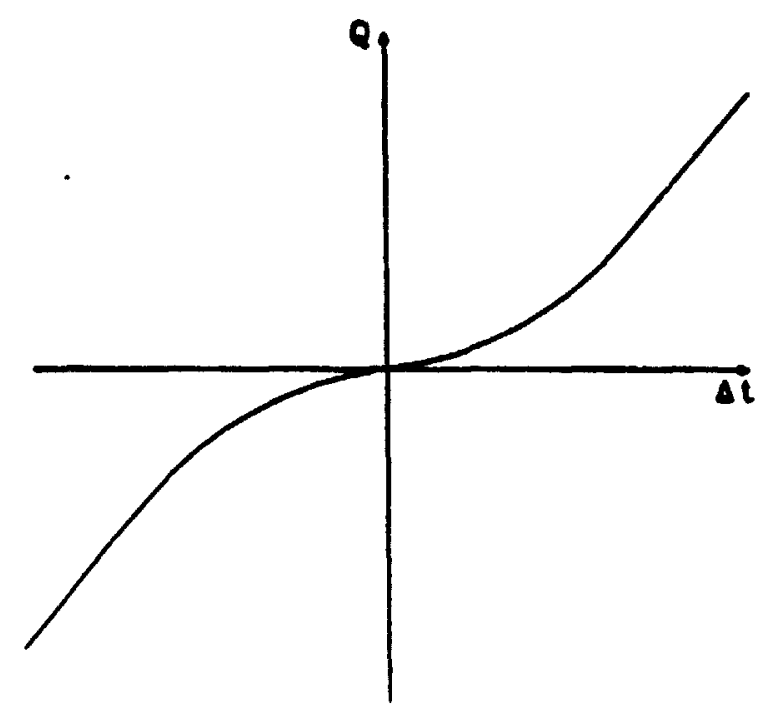

Figure 4.10: Non-Linear Phase Detector Transfer Function for Asymmetrical Propagation Delays

to the feedback elements of the loop filter during this time, are related in a manner similar to the third-order polynomial shown in Figure 4.10. Consequently, if the reference signal contains sinusoidal phase noise at a frequency $\omega_{\theta_{n}}$, we would expect the simulations to show a component at $\omega_{\theta_{n}}$ in the VCO output as well as one at $3 * w_{0}$ and the sampled-data nature of the phase detector is expected to replicate this additional component throughout the spectrum. However, a non-linearity similar to that of Figure 4.10 is difficult to incorporate within the simulation model. The problem is the fact that the non-linearity is associated with the finite rise and fall times of the current being applied to the loop filter. Unfortunately, in its present 
form, the simulation model can not account for this type of signal. As a result, we have used a piecewise-linear approximation shown in Figure 4.11a. The non-linearity is specified in terms of the width of the 'dead zone', $t_{d z}$, and the response of the loop is modelled as follows: since the effect of the non-linearity is to transfer less than the correct amount of charge through the loop filter, the piecewise linear approximation causes the application of current to the loop filter to be delayed from the first edge of the edge pair by $t_{d z}$, as shown in Figure $4.11 \mathrm{~b}$. If the calculated value of $\Delta t_{n}$ falls within the dead zone, no charge is transferred through the loop filter and the decaying exponential transient from the preceding interval continues. An example of this behaviour is shown in Figure 4.12a for an unstressed loop, i.e. where the desired output frequency is equal to the VCO's centre frequency. The width of the dead zone is $40 \mu s$ or $t_{i} / 100$. For comparison, Figure $4.12 b$ shows the behaviour of the same loop but with the non-linearity removed from the phase detector. It is clear that roughly half of the calculated values of $\Delta t$ have fallen within the dead zone and, consequently, these samples of $\theta_{d}(t)$ have no effect on the behaviour of the system.

It is interesting to note that, of the two responses shown in Figure 4.12, the one corresponding to the non-linear phase detector appears to be 'cleaner', i.e. it contains fewer transients, with the expected result being reduced phase noise at the output. This is confirmed by the distributions of $S_{w_{\text {ino }}}$ shown in Figure 4.13a and Figure 4.13b 


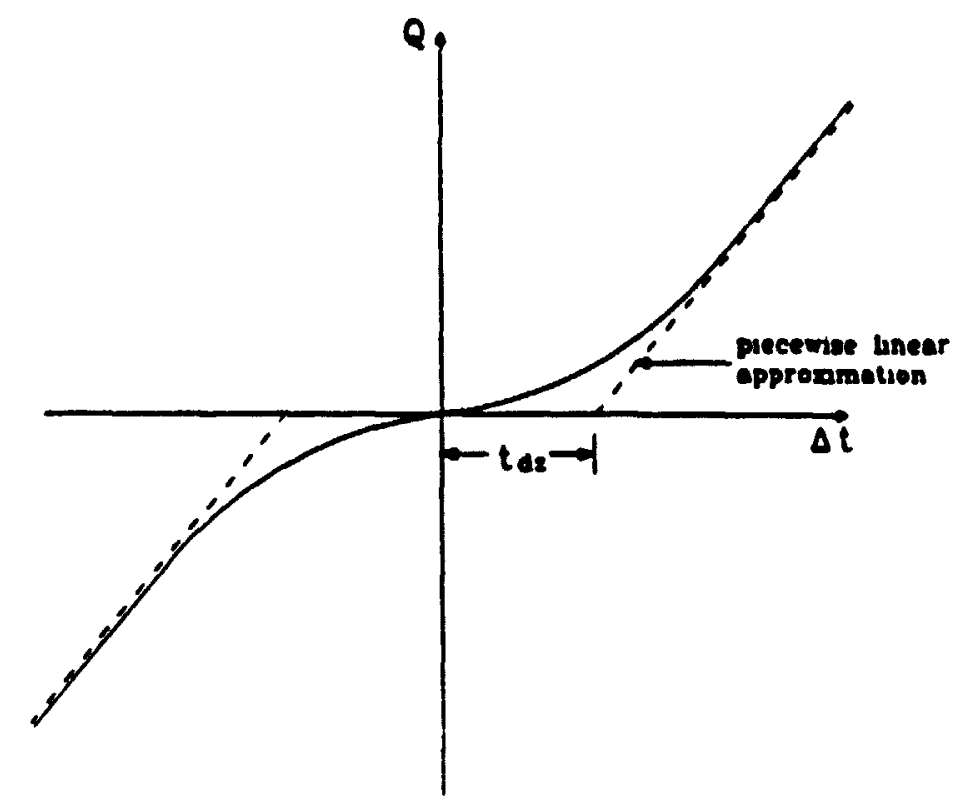

(a)

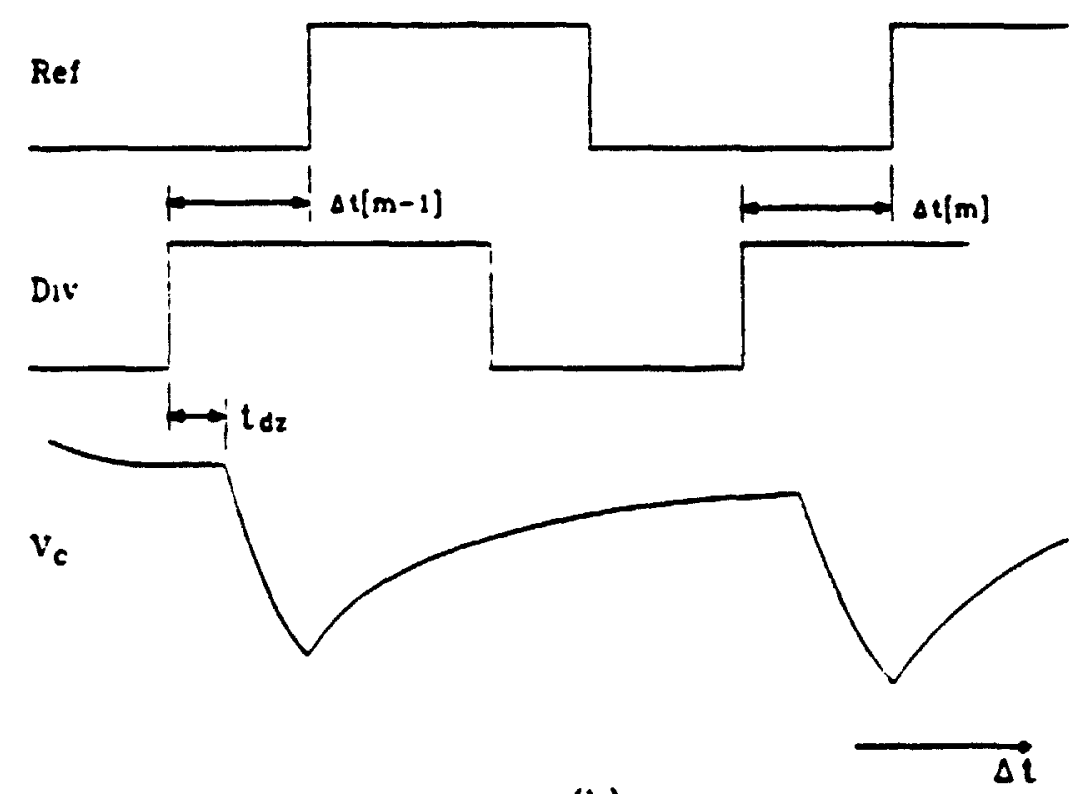

(b)

Figure 4.11: Modelling the Propagation Delay Non-Linearity (a) Piecewise Linear Approximation (b) Time-Domain Behaviour 


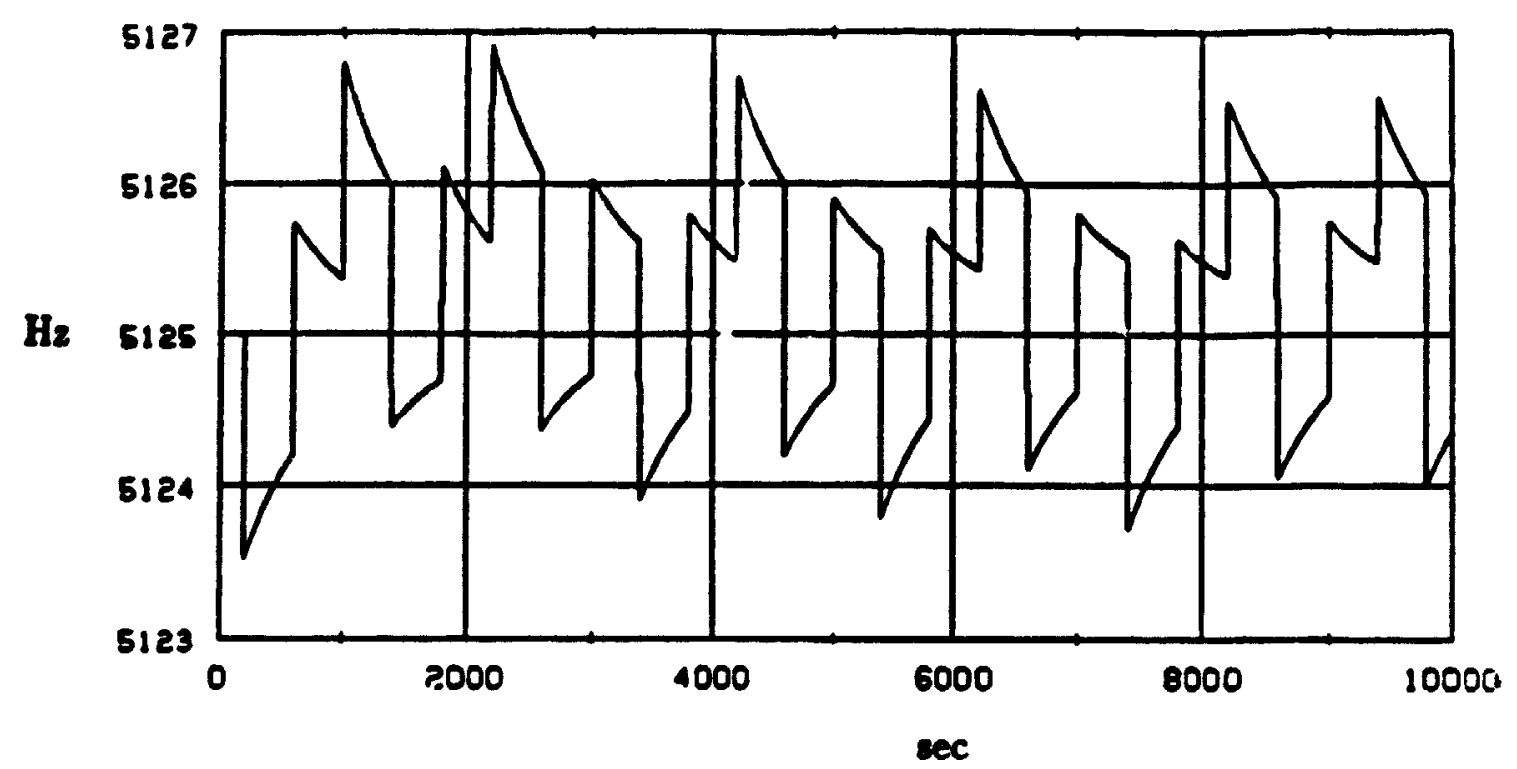

(a)

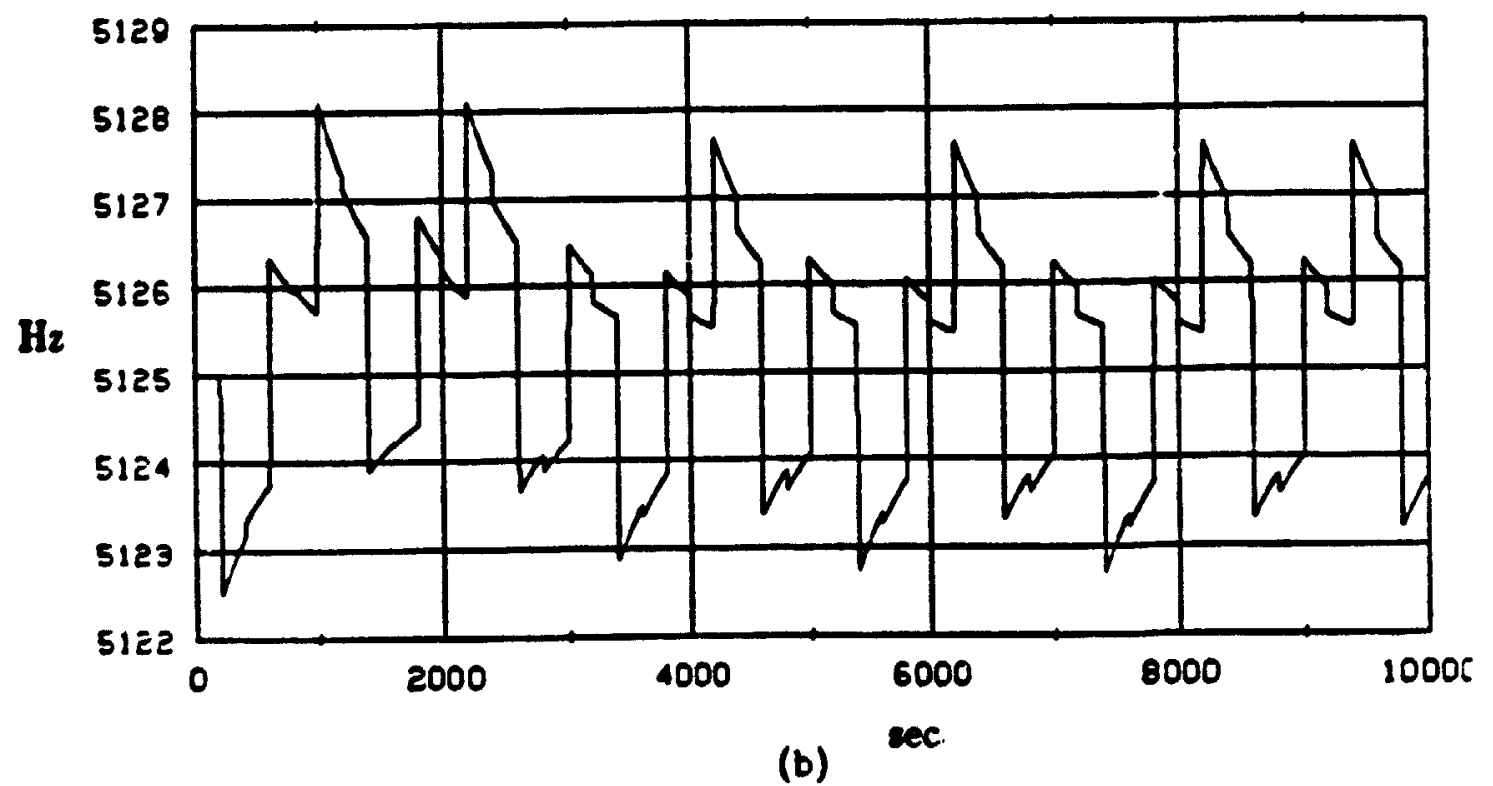

Figure 4.12: $\omega_{\text {ino }}(t)(a) t_{d s}=40 \mu s$ (b) $t_{d s}=0$ 
but is contrary to what we would intuitively expect. Generally, a non-linearity will act to raise the level of unwanted signals and the fact that this is not the case here suggests that modelling this non-linearity in terms of a 'dead zone' is inappropriate. In fact, this conclusion becomes obvious when we look more closely at the effect of the 'dead zone'. It turns out that by reducing and, in some cases, eliminating the effect of a phase difference between Ref and Div, we have actually reduced the loop bandwidth and, with this in mind, the decrease in output phase noise comes as no surprise. However, it is doubtful that the same effect would be observed in a practical frequency synthesizer in the presence of this type of non-linearity. Consequently, if this non-linearity was to be re-examined in the future, a more detailed means of incorporating it within the simulation structure would be required.

Now consider a non-linear phase detector transfer function caused by an asymmetry between current sources in the charge-pump. If the values of the current sources supplying positive charge to and drawing positive charge from the loop filter are denoted as $I_{p p}$ and $I_{p m}$ respectively, the relationship between $Q_{n}$ and $\Delta t_{n}$ when $I_{p p}=0.5 * I_{p}$ and $I_{p m}=1.5 * I_{p}$ is shown in Figure 4.14. $I_{p}$ is the nominal value of the current sources. Figure 4.15 shows the effect of this non-linearity on the spectrum of the instantaneous output frequency when the reference signal contains sinusoidal phase noise at $160 \mathrm{~Hz}$. The important point to note is that this spectrum 


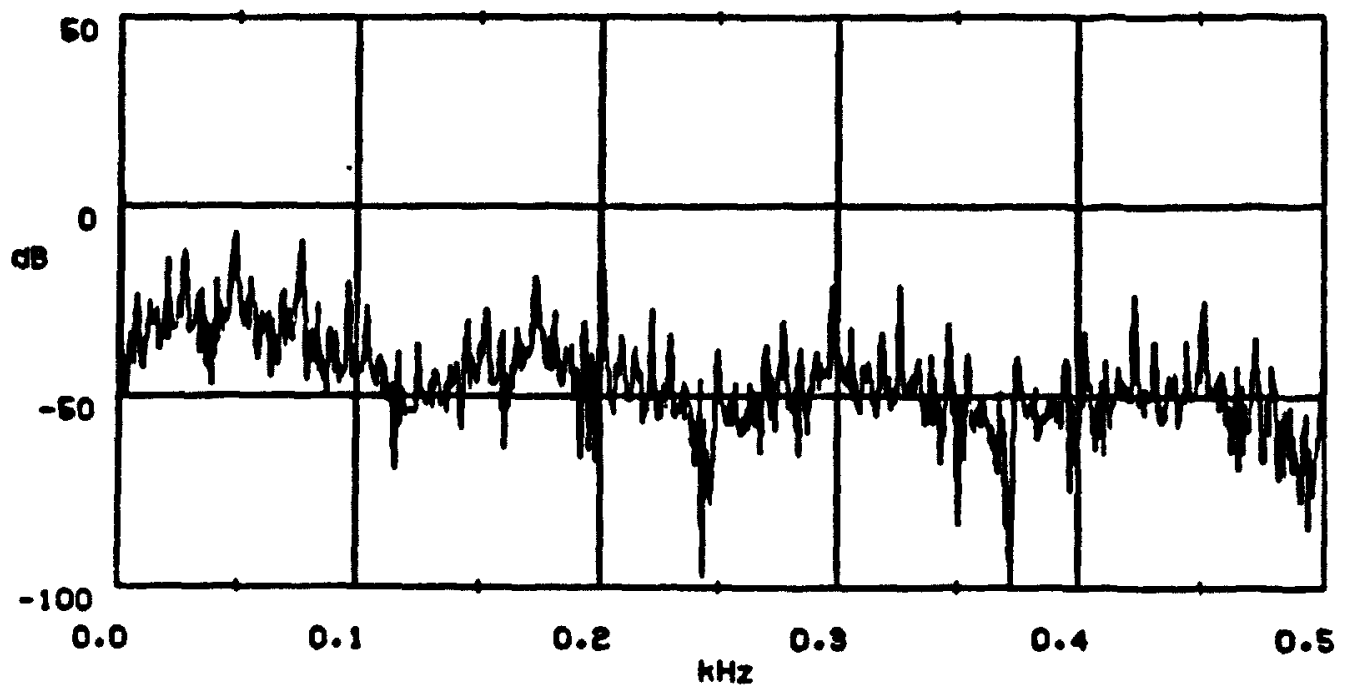

(a)

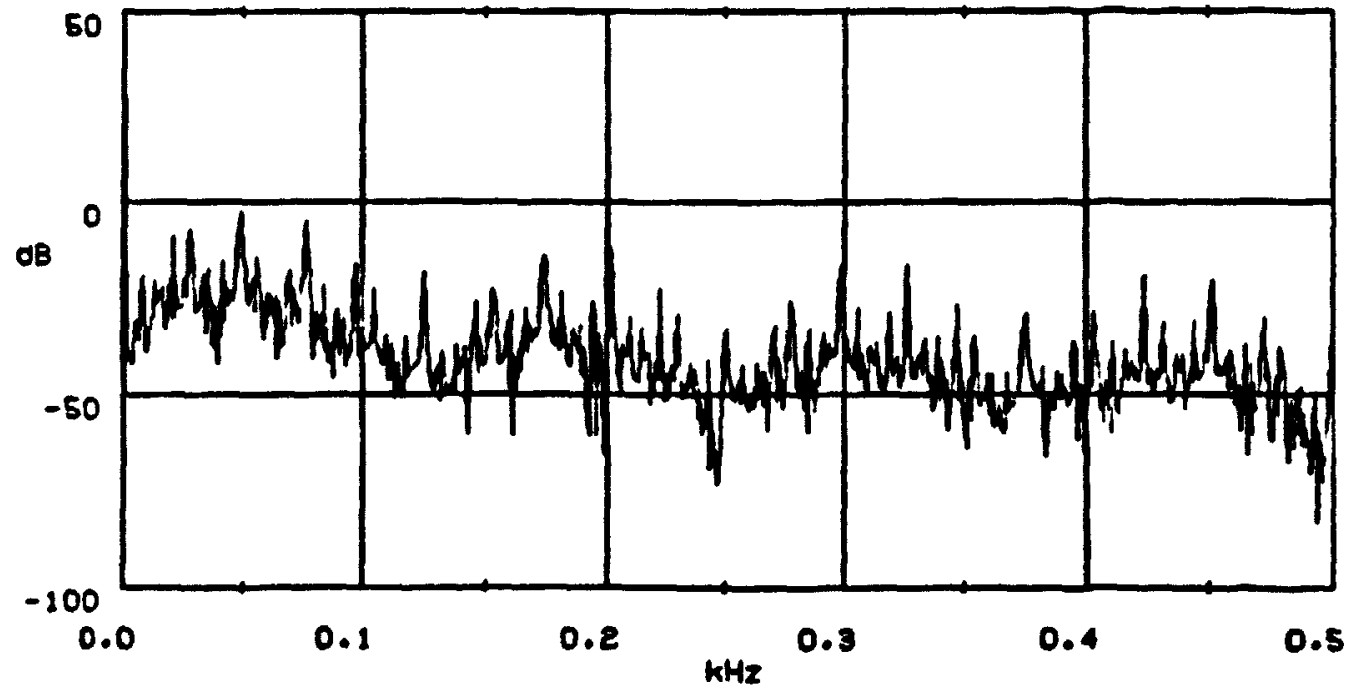

(b)

Figure 4.13: $S_{\omega_{1, n}}(w)(a) t_{d s}=40 \mu s$ (b) $t_{d z}=0(P=1140)$ 
shows a periodicity at $50 \mathrm{~Hz}$; we believe the explanation for this behaviour is that the non-linearity is responsible for the generation of the third harmonic of the phase noise at $300 \mathrm{~Hz}$. When this component is sampled at the frequency of the reference signal, the result is a replication of that component to $50 \mathrm{~Hz}$. Then, in more general terms, we can say that components generated by this non-linearity, near multiples of the reference frequency, may be replicated within the loop bandwidth and possibly within the region of noise-shaping around the average output frequency.

Finally, we noted earlier that additional spectral components may occur near the average output frequency due to intermodulation of the spectrum of the input phase difference. Figures $4.16 \mathrm{a}$ and $4.16 \mathrm{~b}$ show more detailed views of the region of noiseshaping near dc, i.e., around the average output frequency, with the current source non-linearity $\left(I_{p p}=0.8 * I_{p}\right.$ and $\left.I_{p m}=1.2 * I_{p}\right)$ and without, but with no input phase noise. In comparing the two figures, we can see that there is a noticeable increase in the level of output phase noise at frequencies near dc. Unfortunately, at present we have no way of determining whether the increase is primarily due to replication of the components generated by the non-linearity or to intermodulation of the input phase noise. The problem is that, since the sampled-data nature of the charge-pump phase detector is implied by its basic operation, we have no way of performing simulations where spectral replication of $\theta_{d}(\omega)$ is not present. Nevertheless, the fact that there 


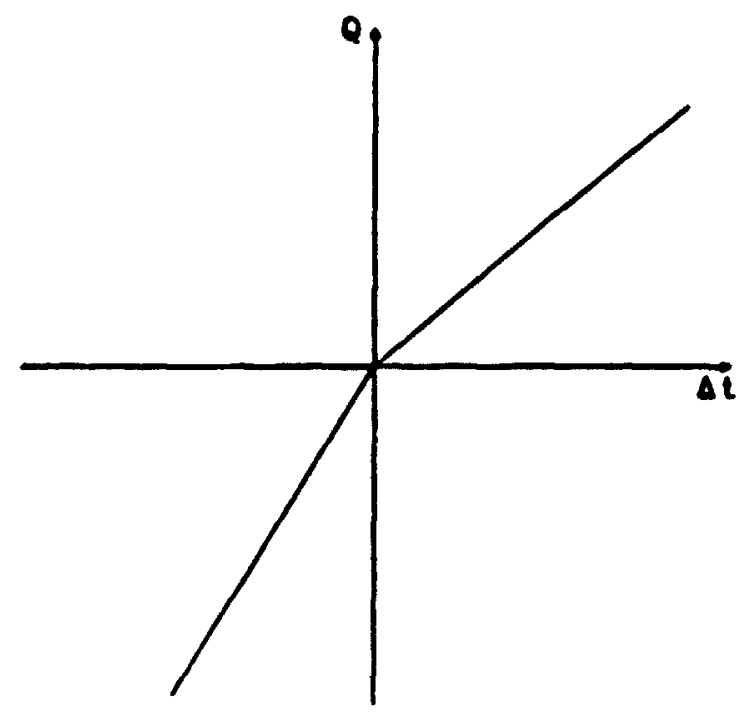

Figure 4.14: Phase Detector Transfer Punction Including Current Source Non-Linearity

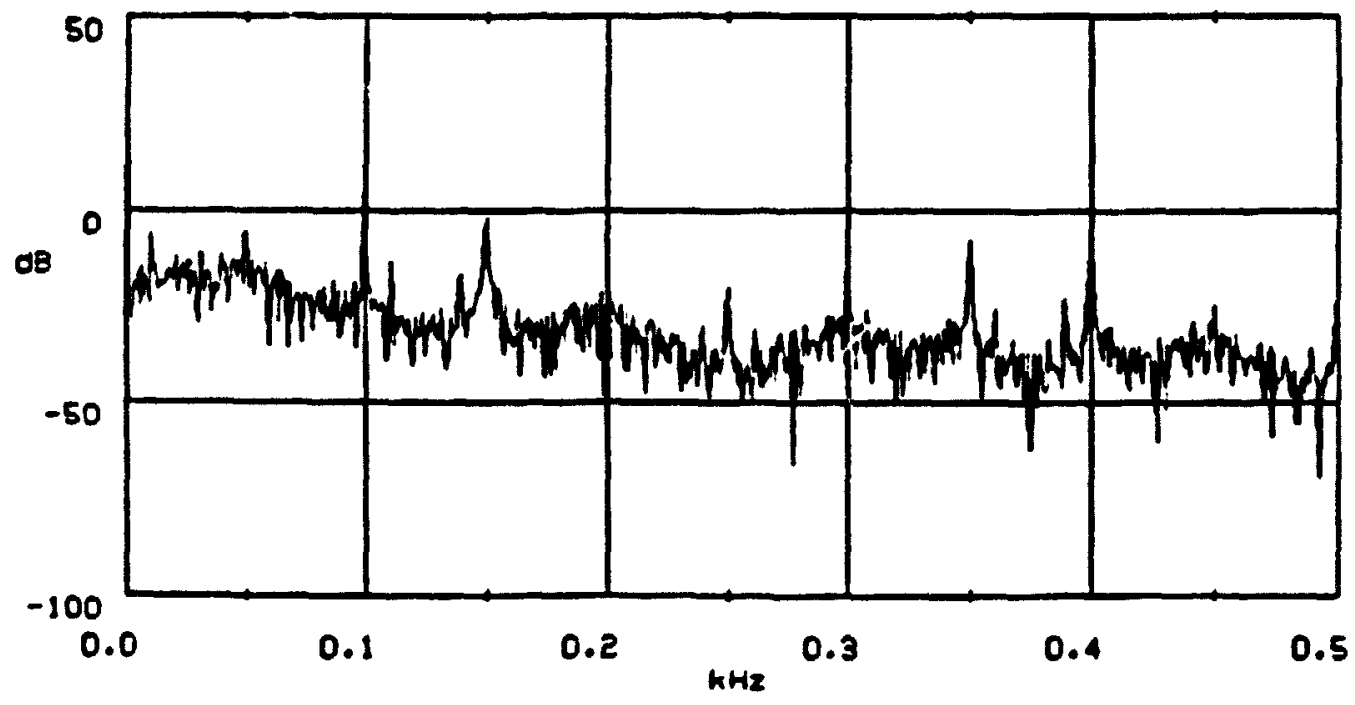

Figure 4.15: $S_{\omega_{100}}(\omega)$ - Phase Detector Includes Current Source Non-Linearity, $\omega_{0}=$ $0.4 * w_{1} \quad(P=1140)$ 
is a measurable increase in phase noise suggests that this might be an area requiring further investigation.

\subsection{Summary}

In this chapter, we have examined the simulated behaviour of a fractional- $N$ frequency synthesizer under a number of different conditions. We have demonstrated the advantages of a simulation structure for evaluating the general behaviour of this device and, in particular, for examining the sampled-data and non-linear aspects of the charge-pump phase detector. As expected, the sampled-data nature of a chargepump phase detector is responsible for the repiication of $\theta_{d}(\omega)$ around all multiples of the reference frequency. In general, this replication will tend to raise the level of spurious signals at the output of the VCO with the result being a decline in the spectral purity of the output signal. The non-linear aspects of typical charge-pump phase detectors can contribute to this decline through replication of the components generated by the non-linearity or through the generation of intermodulation products near the average output frequency. 


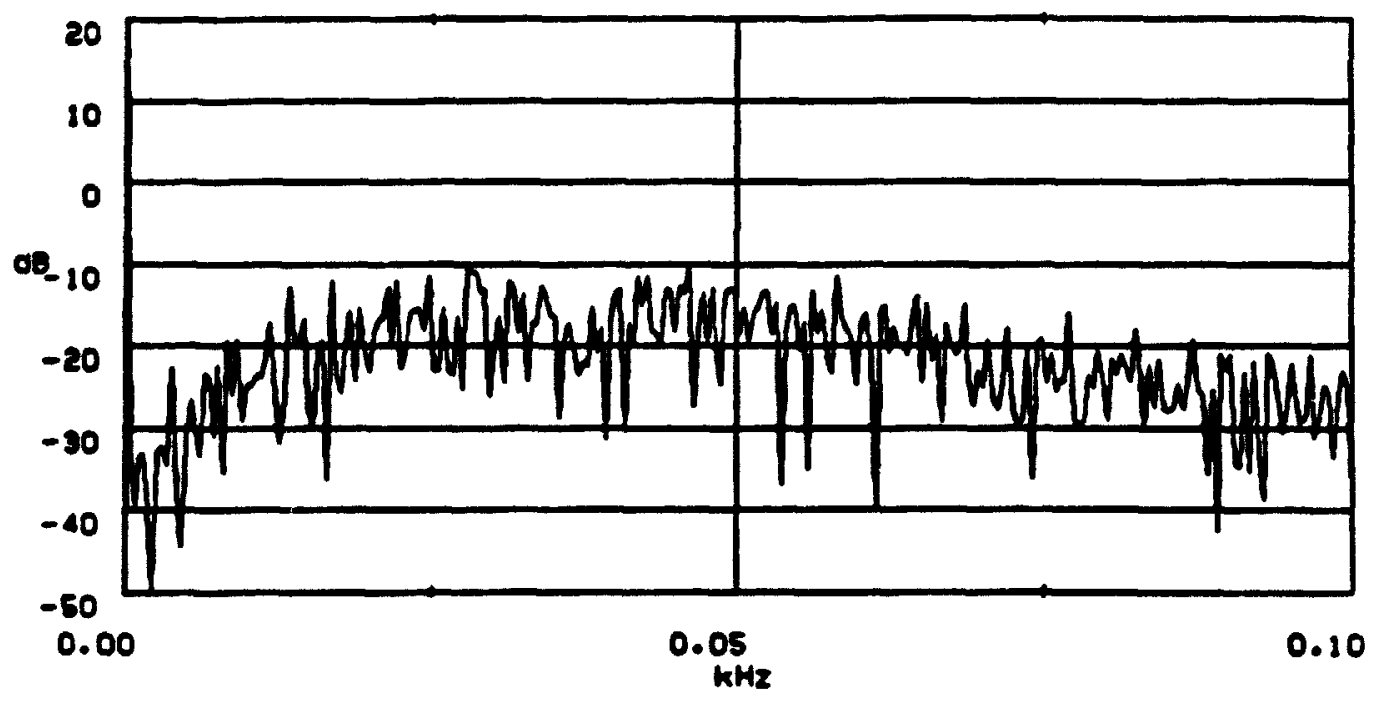

(a)

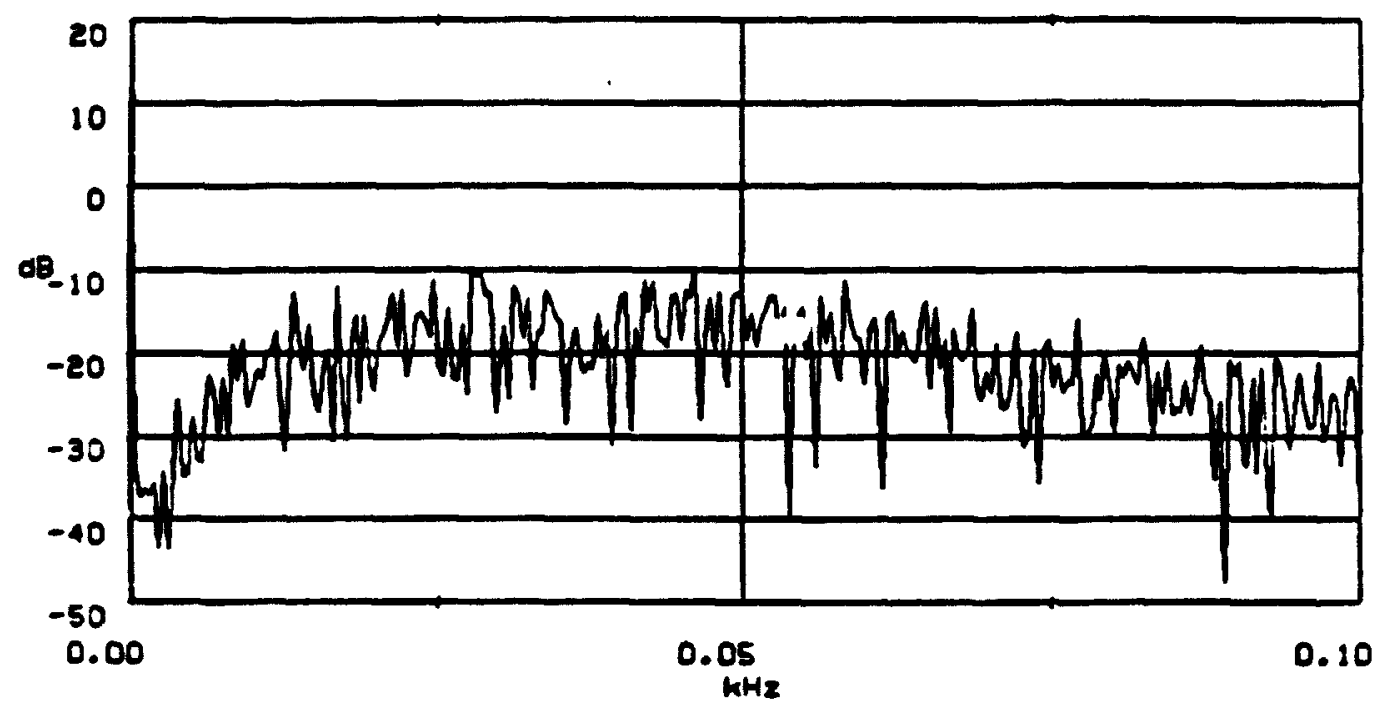

(b)

Figure 4.16: $S_{\omega_{\text {woo }}}(\omega)(a) w_{.}$b Current Source Non-Linearity (b) without Current Source Non-Linearity $(P=1140)$ 


\section{Chapter 5}

\section{Conclusions}

In summary, the general objective of this thesis was to develop a better understanding of the behaviour of a monolithic frequency synthesizer implemented as a phase-locked loop with a charge-pump phase detector, with particular emphasis on the issues related to a monolithic implementation. We have focused on the consequences of the sampled-data and non-linear aspects of a charge-pump phase detector because of the possibility that spectral replicas and spurious components will lead to an increase in the level of phase noise near the average output frequency, thereby imposing a limitation on the overall performance of the loop. Consequently, the aim of this thesis was to develop a framework for determining whether this increase presents a problem for practical, monolithic fractional-N frequency synthesizers. 
The first step towards this goal was the development of the simulation structure described in Chapter 2. We determined that the conventional means of modelling a phase-locked loop tended to obscure important elements of the behaviour of the loop and that a new approach was required. The basis of this approach is a representation of the time-domain behaviour of the loop which can be evaluated over a number of cycles of the reference input. A Discrete Fourier Transform is then used to convert the variations in the instantaneous output frequency to the frequency domain.

Using this simulation structure, we were able to verify that the spectrum of the input phase difference is replicated around all multiples of the reference frequency. When more extensive simulations are performed and the results are compared with the performance of a continuous loop, we should be able to judge if the spectral replicas lead to a significant liscrease in the level of phase noise near the average output frequency. This is also true when considering the effect of a typical, non-linear phase detector. We saw that an asymmetry between the current sources in the charge-pump is capable of contributing additional phase noise near the average output frequency, either through intermodulation or replication of the input spectrum. Based on more complete simulations we should be able to develop some idea of the magnitude of the increase and whether one of the sources, spectral replication or intermodulation, dominates. Generally, the simulation structure provides a means of evaluating the 
output characteristics of a particular loop under a variety of conditions.

If further research is to be carried out in this area, the incentive will likely come from specific applications where the effect of the spectral replica.ion and non-linearity is comparable to the other sources of phase noise within the loop. Generally, it seems that additional research will be required simply to increase the level of detail associated with a particular application. This increased detail will develop through incorporating numerical information, derived from actual realizations of key components, into the simulations and through more extensive simulations, where the number of calculations of the instantaneous output frequency is increased. As well, the fact that, at present, we have no reference against which these simulation results can be compared suggests that there could be value in the development of a simulation structure similar to the one presented here, but based on a multiplier as the phase detector.

However, as noted early in this thesis, being able to simulate ibe behaviour of a circuit is of limited value unless we are also able to explain this behaviour. Consequently, it scems appropriate that we also attempt to develop an analytical representation of a charge-pump phase detector, with the ultimate goal being a relationship between the spectrum of the input phase difference aud the spectrum of the output signal. Once this relationship is established, we should be in a better position to determine 
whether or not the second-order aspects of a charge-pump phase detector present a problem for practical fractional-N frequency synthesizers.

To conclude, this thesis has succeeded in describing and explaining certain secondorder mechanisms which could have an important impact on the performance of monolithic, fractional-N frequency synthesizers. No attempt to be precisely quantitative was made because a high quality VLSI implementation has not yet been tested. However, once the improved numerical information is available, the simulation techniques developed here can be expanded so as to better characterize the mechanisms described here and to support the application of monolithic, fractional-N frequency synthesizers. 


\section{Bibliography}

[1] T.A.D. Riley, M.A. Copeland, and T.A. Kwasniewski, Sigma Delta Modulation in Fractional-N Frequency Synthesis, VLSI in Communications Group of TRIO, Department of Electronics, Carleton University, Ottawa, Ontario, 1988.

[2] Isabelle Jaquet, A Comparison of Phase Noise Control Techniques used in High Resolution High-Speed Frequency Synthesizers for Mobile Radio Transceivers, M.Eng. Thesis, Carleton University, Ottawa, Ontario, 1990.

[3] Ulrich L. Rohde, Digital PLL Frequency Synthesizers, Prentice-Hall, Englewood Cliffs, 1983.

[4] Motorola Semiconductor Application Note, Operation of the MC145159 PLL Freguency Synthesizer with Analog Phase Detector, Motorola Inc., 1987. 
[5] D. Asta and D.N. Green, Analysis of a Hybrid Analog/Switched-Capacitor PhaseLocked Loop, IEEE Transactions on Circuits and Systems, vol.37, pp. 183-197, Feb. 1990.

[6] Floyd M. Gardner, Charge-Pump Phese-Lock Loops, IEEE Transactions on Communications, vol. COM-28, pp. 1849-1858, Nov. 1980.

(7) Roubik Gregorian and Gabor C. Temes, Analog MOS Integrated Circuits for Signal Processing, John Wiley \& Sons Inc., New York, 1986.

[8] William F. Egan, Frequency Synthesis by Phase Lock, John Wiley \& Sons Inc., New York, 1981. 


\section{Appendix A Source Code}

(complied under SunOs 4.0)

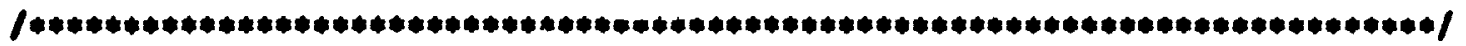
1* Iractional-y irequency oyathosis vith altiple sappling in ach period $* 1$ 1* third-order. type-2 100p f* difider coatrolled by second-order alga-delta nodulator 1* don-Iinearity added to phase detector

$1 *$

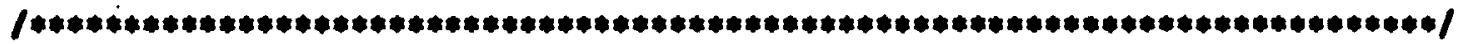

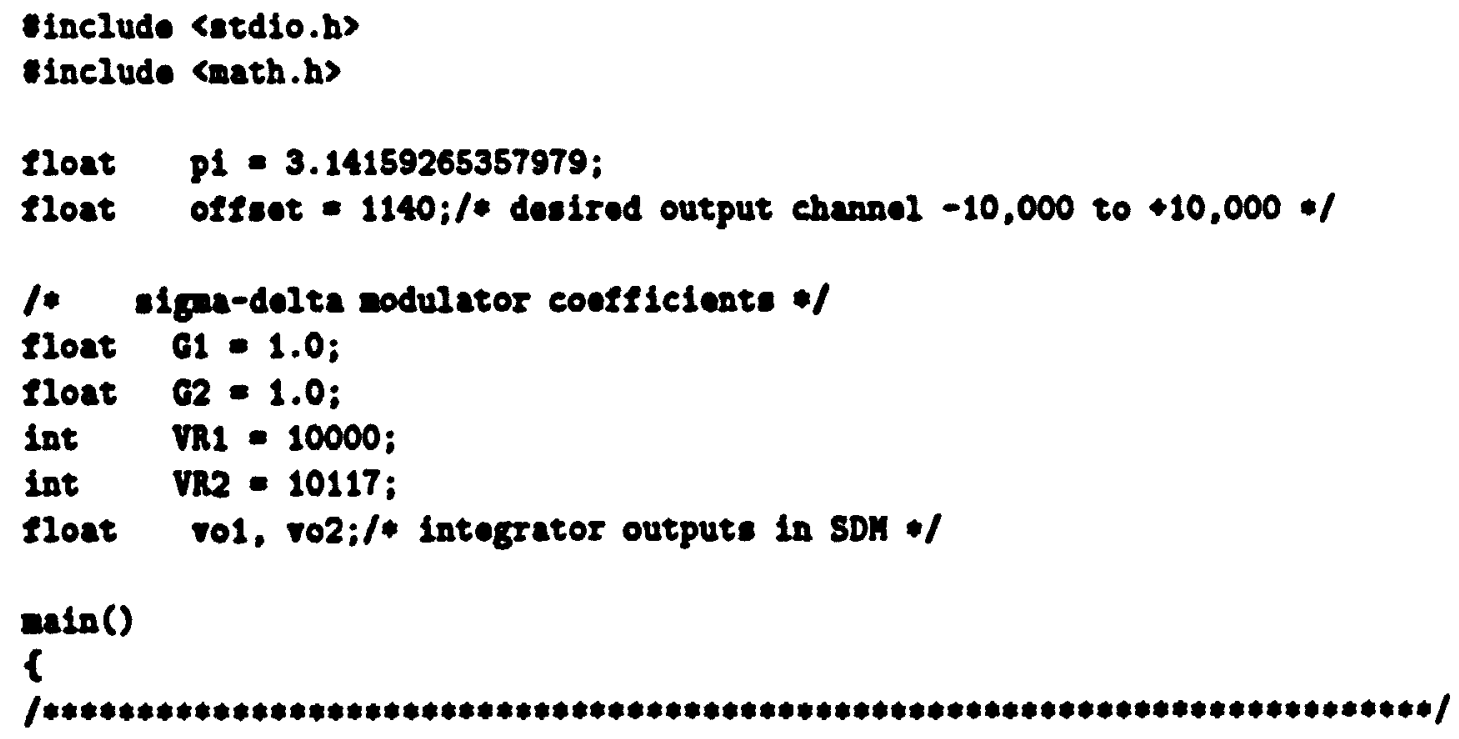




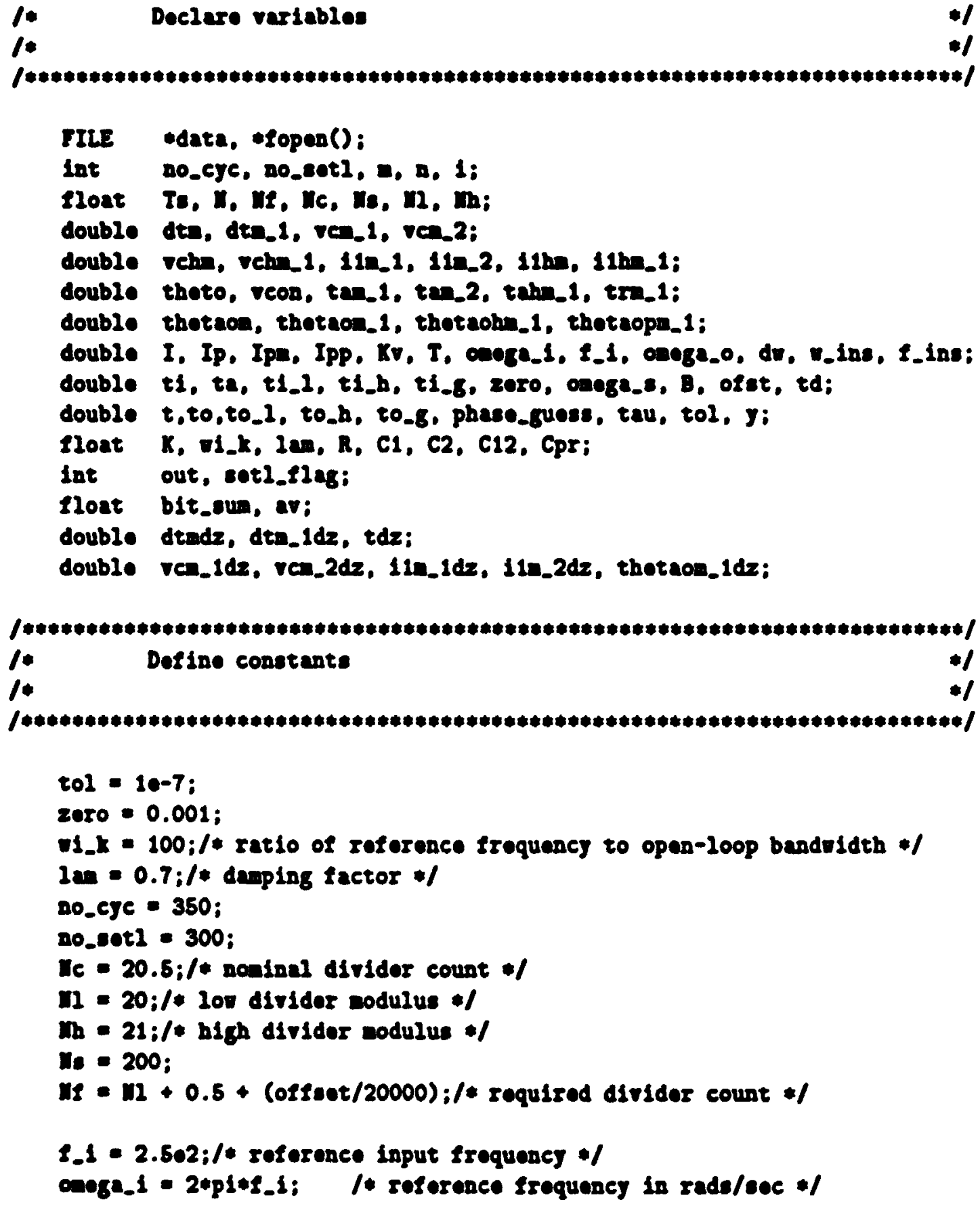




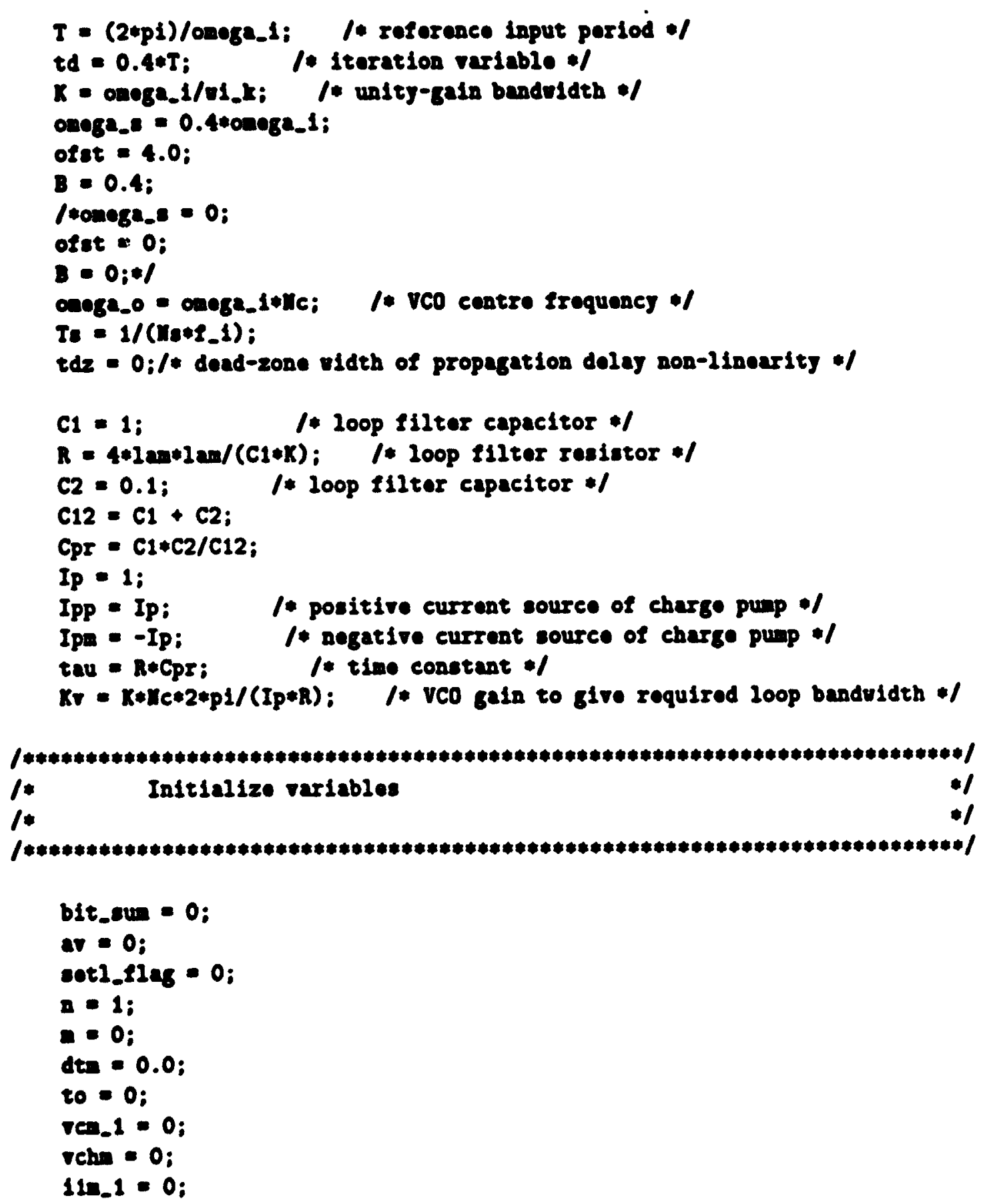


$11 \mathrm{hn}=0$;

ten_1 $=0$;

thotece $=0$;

ea 0;

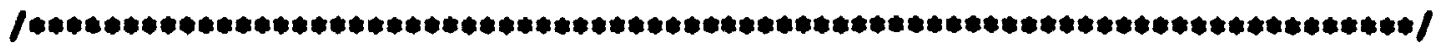

$1 *$

opon dete 2110

1

10

4

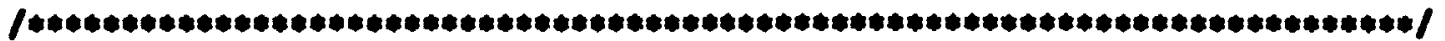

11 ((data = Lopen("Irac2.dat". "v")) $=0$ muLL)

printe ("Can't open frac2.datla"):

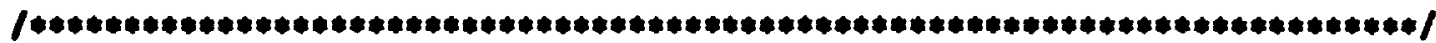
1. Start alge-delta nodulator and run for 16 cjeles */ 10

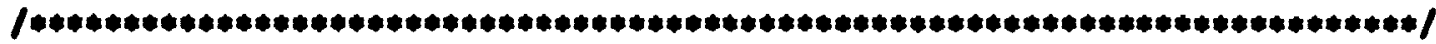

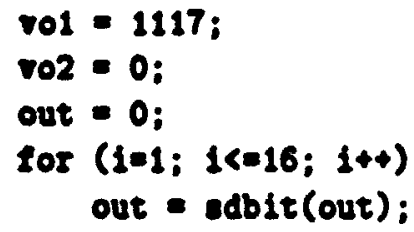

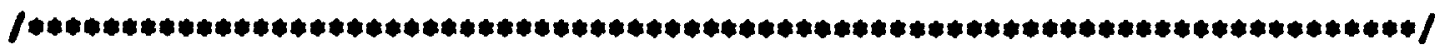
F Main sinulation loop - calculate dta, difieronce in tin beteen */ f* edzer of hot and DIV. besed on provious value *f 10

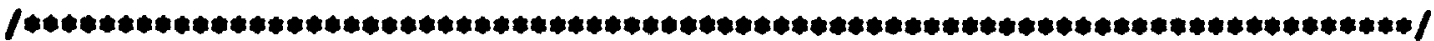

do

I

11 (eotr_tles $=0)$

f* allor tine for loop to oottle to oteady otate $+/$

18 ( $100.00 t 1)$

1* eoteling poriod is complote *f 


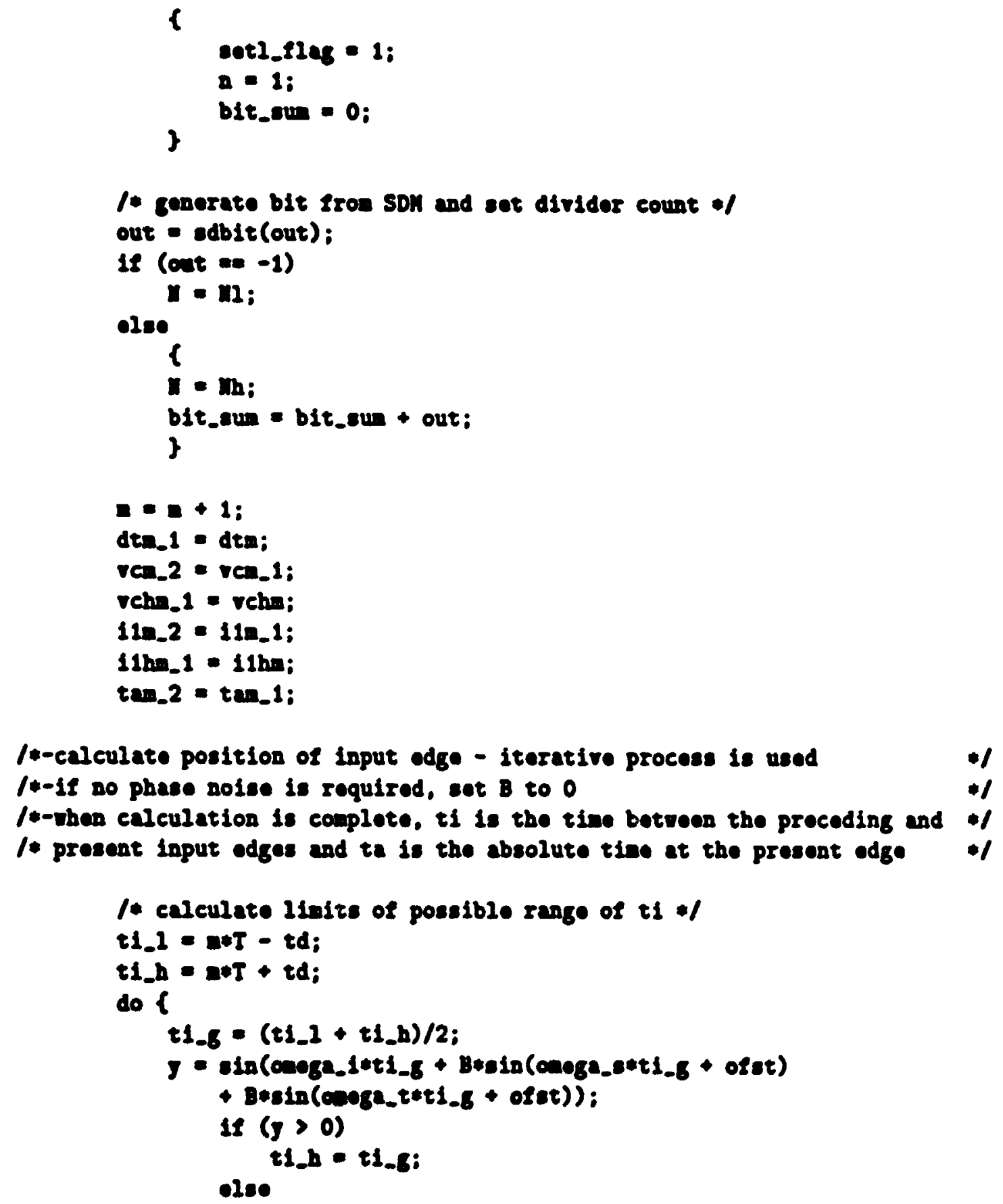

1*-calculate position of input edge - iterative process is used /*-if no phase noise is required, set B to 0

/*-then calculation is complote, ti is tho tian botween the proceding and *I 1* present input edges and to is the absolute tine et the present edge 


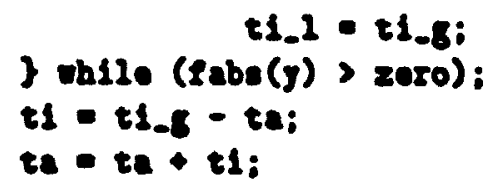

1. calculate porlod of divider output, to, based on provious value of dte *f

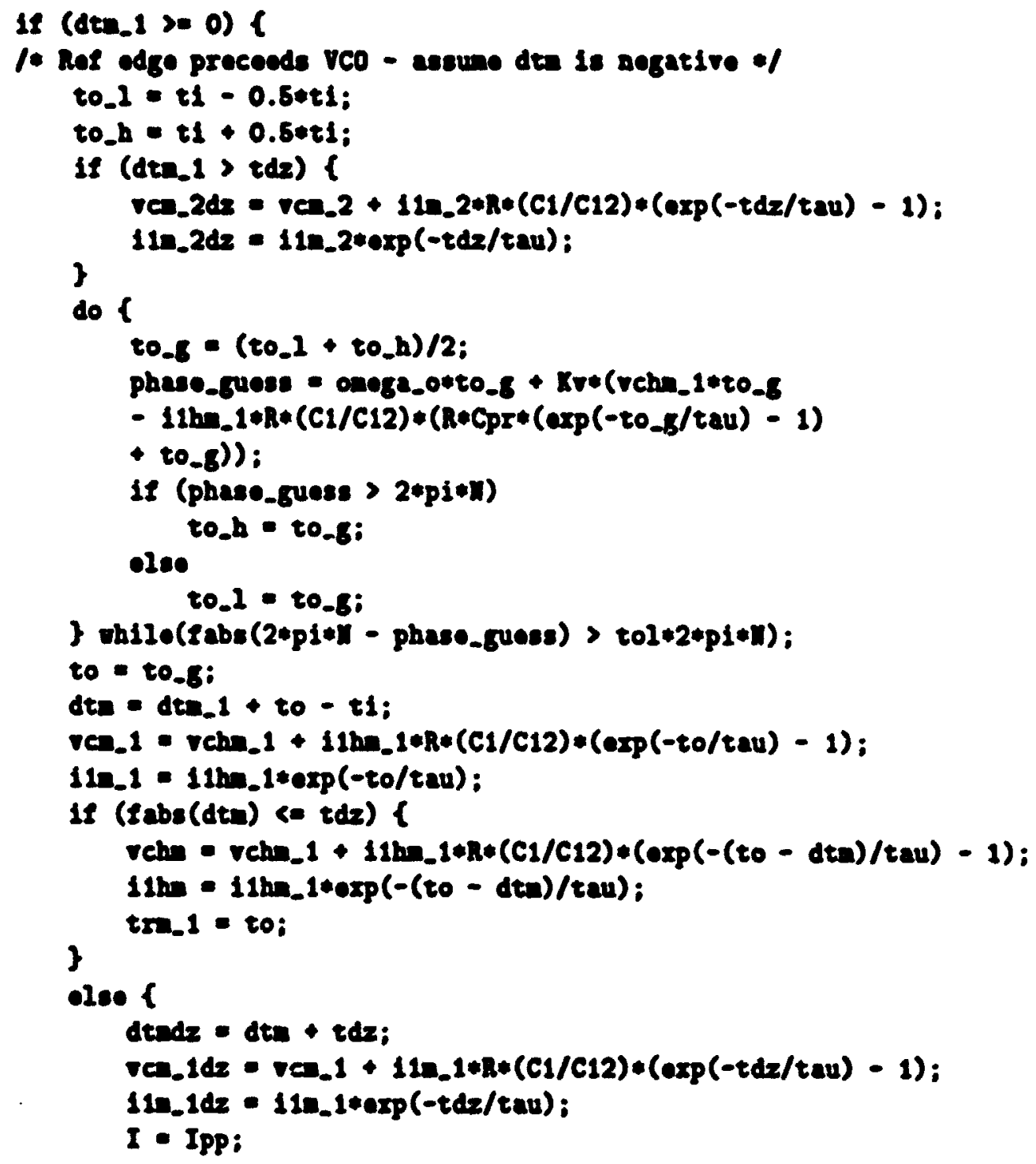




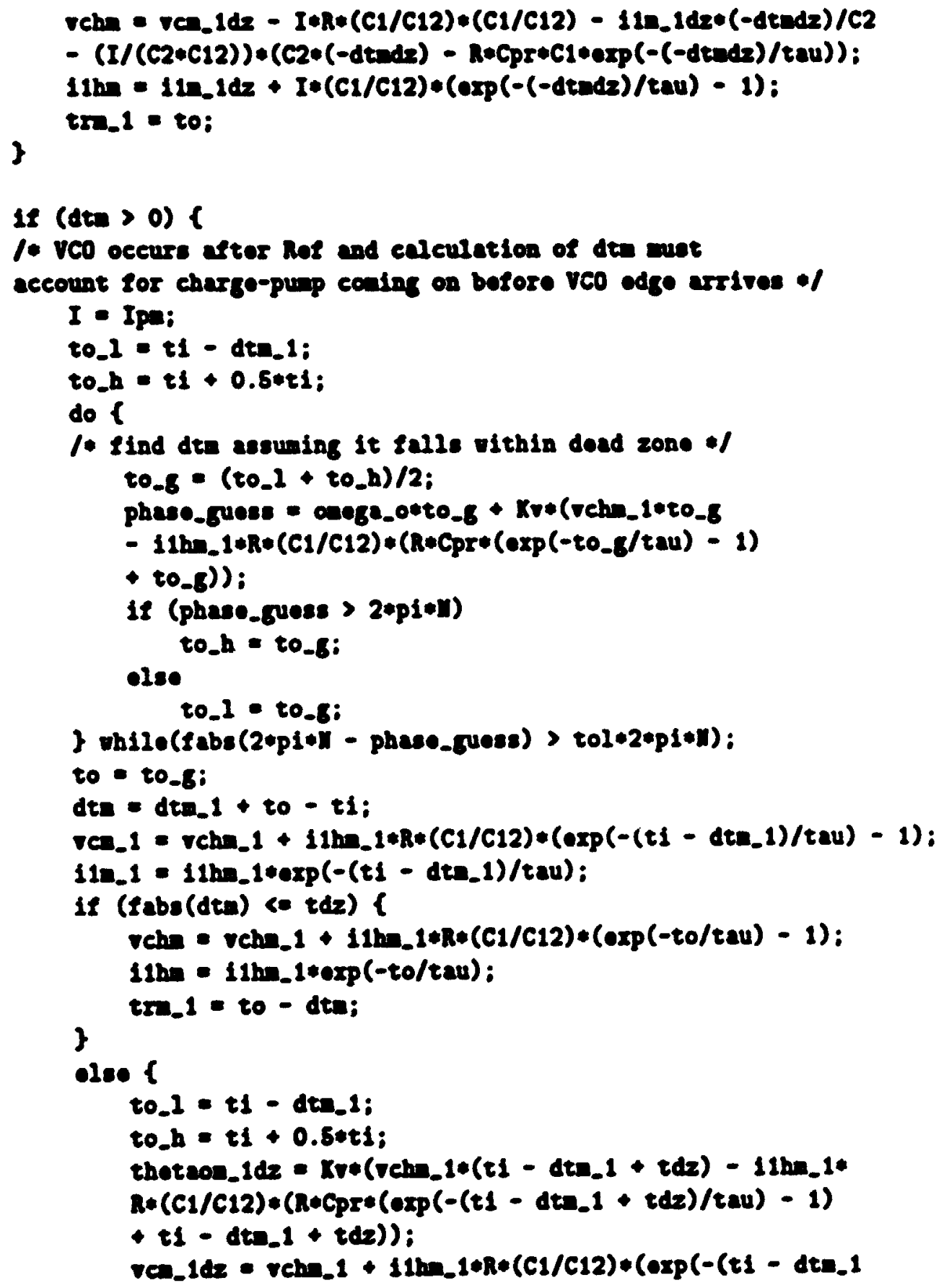




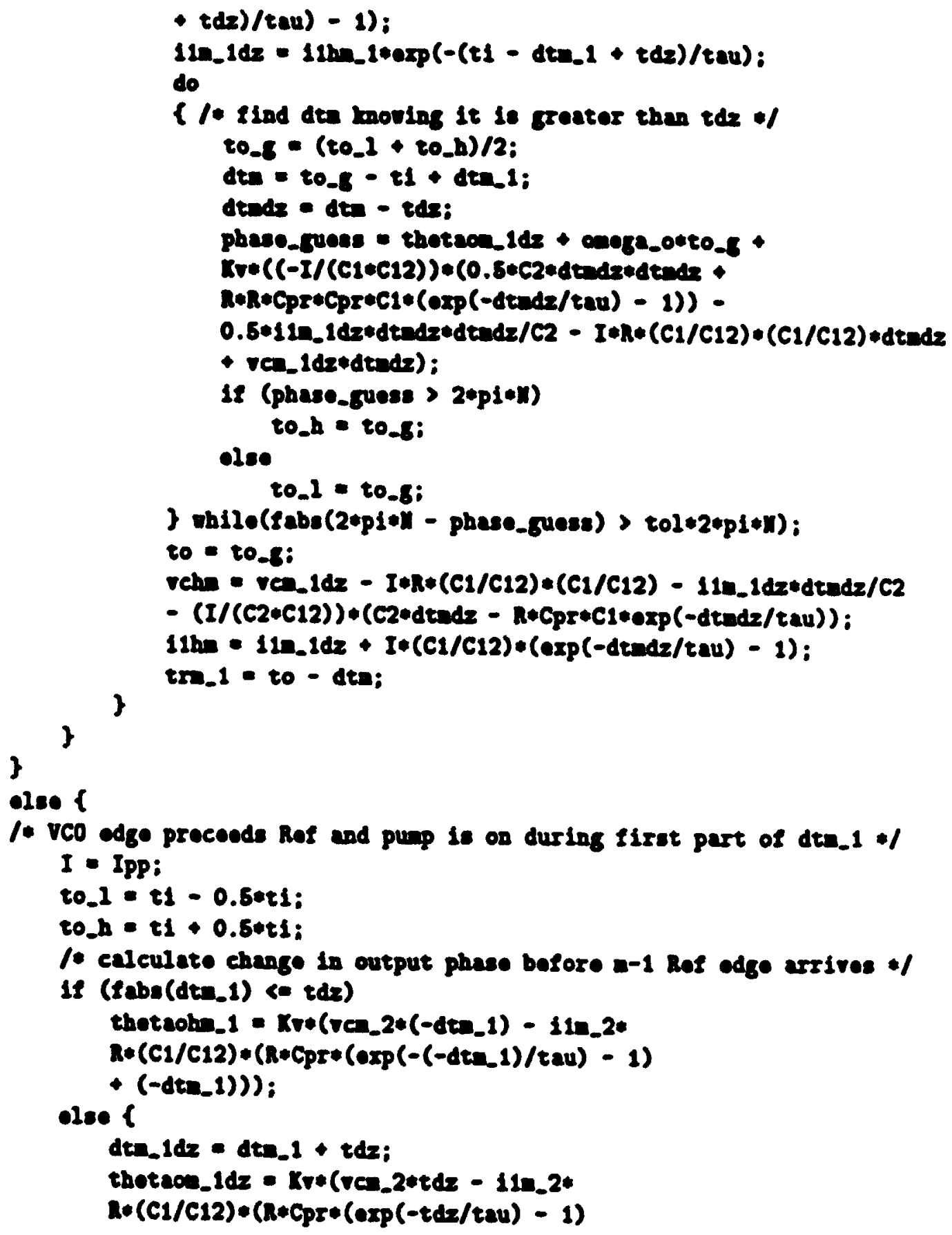




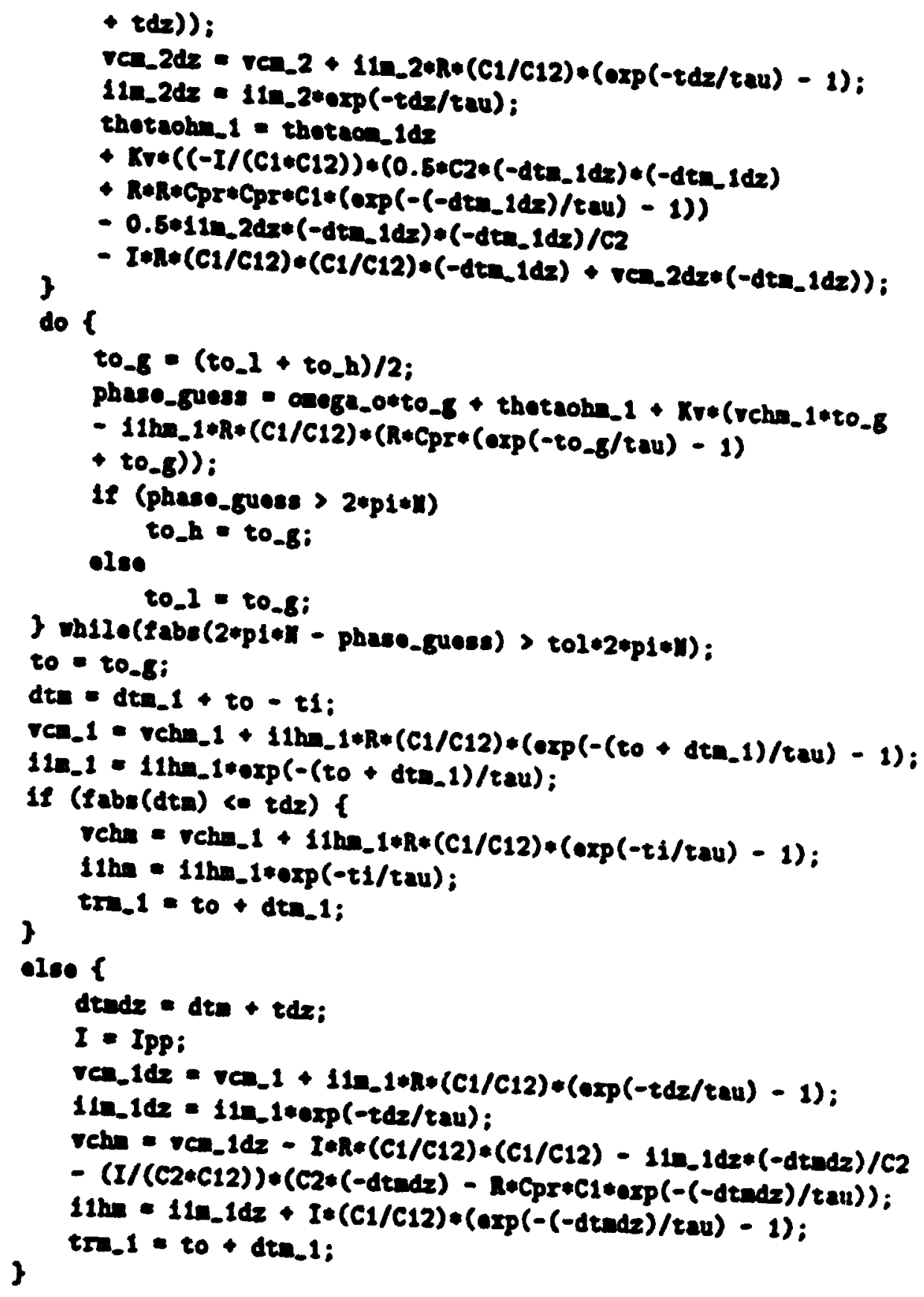


19 (dte > 0 ) 1

1. VCO leade Rot in a-1 interval but lage Rot in a-th Interval of I - IPP:

to_l $=t 1$ - dta_1:

to_h $=t 1+0.5 * t 1 ;$

$1 \cdot$ thot:oohn_1 $=x \nabla *\left((-I /(C 1 * C 12)) *\left(0.5 * C 2 *\left(-d t n_{2} 1\right) *\left(-d t m_{-} 1\right) *\right.\right.$ $R * R * C p r * C p r * C 1 *(o x p(-(-d t=-1) / t a u)-1))-$

$0.5 * 12 I_{-} 2 *\left(-d t n_{-} 1\right) *\left(-d t n_{-1}\right) / C 2-I * R *(C 1 / C 12) *(C 1 / C 12) *\left(-d t m_{-} 1\right)$ + Van_2*(-dta_1)); *I thotaon_1 * thotaohn_1 + Xv*(rchn_1*t1 - 11bm_1*

$R *\left(C 1 / C_{12}\right) *\left(R * C_{p r} *(\exp (-t 1 / t a v)-1)\right.$

$+(1))$ :

$\operatorname{ven}=1=\operatorname{vehn}-1+11 \mathrm{hn}+1 * R *(C 1 / C 12) *(\operatorname{oxp}(-t i / t a u)-1)$;

11m_1 = 11hm_1*axp $(-t i / t a u)$;

$I$ Ipm;

do $t$

to-g $=\left(t 0_{-} 1+t 0_{-} h\right) / 2$;

dta = to.-5 - $t 1+d t n_{-} 1$;

phase_gunse = thotaon_1 + omoga_o*to_g + Kr*(vem_l*dtm

- 11E_L $\left.1+R *(C 1 / C 12) *\left(R * C_{p r} *(\exp (-d t a / t a u)-1)+d t m\right)\right)$;

12 (phase-guese > 2*p1*11)

to.h $=$ to.-8;

0180

to.1 = to.-8;

) uhile(fabs (2*pi*ll - phase-guess) > tol*2*pi*II);

to $=$ to-8:

If (fabs(dtz) $c=t d z)$ I

vehn $=$ vchn_1 $+11 \mathrm{hm}, 1 * R *(C 1 / C 12) *(\exp (-(t 1+d t \mathrm{~m}) / \mathrm{tau})-1)$;

IItw $=11 \mathrm{hm}$ _l*oxp $(-(t 1+\mathrm{dtm}) / t a u)$ :

了

trm_1 - ti; ;

218 ᄂ

to_1 $=t 1$ - dta_1;

to_h $=t 1+0.5 \pm t 1 ;$

thotson_1dz = thotaon_1 + Kv*(vam_1*tdz - 11m_1*

$R *(C 1 / C 12) *\left(R * C_{p r} *(\exp (-t d z / t a u)-1)\right.$

+ t(dz)):

ven_1dz $-\mathrm{ven}, 1+11 n_{-} 1 * R *(C 1 / C 12) *(\exp (-t d z / t a u)-1)$;

11._1dz = 11n_1*exp $(-t d z / t a u)$; 


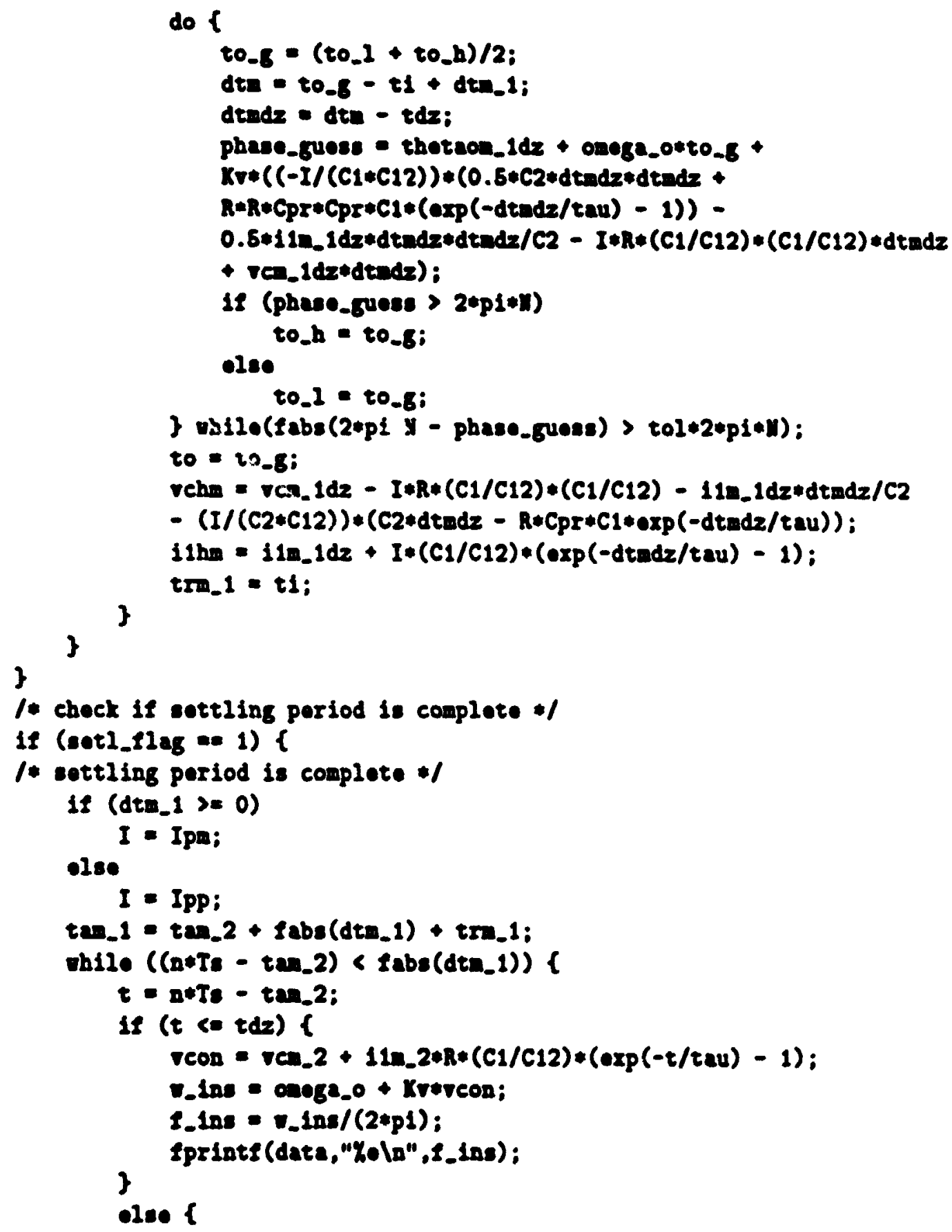




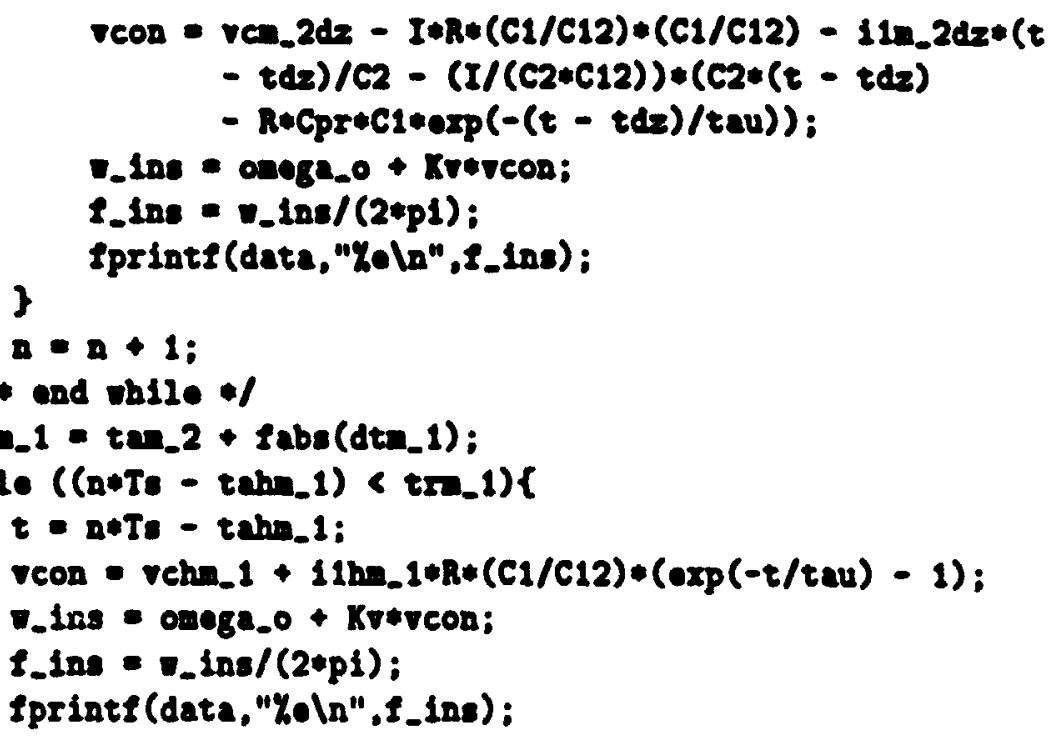




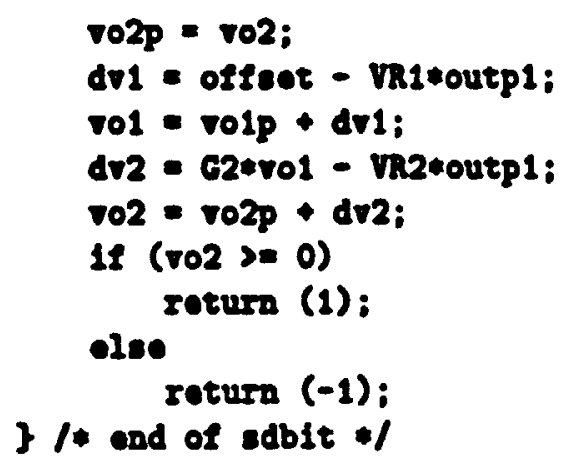



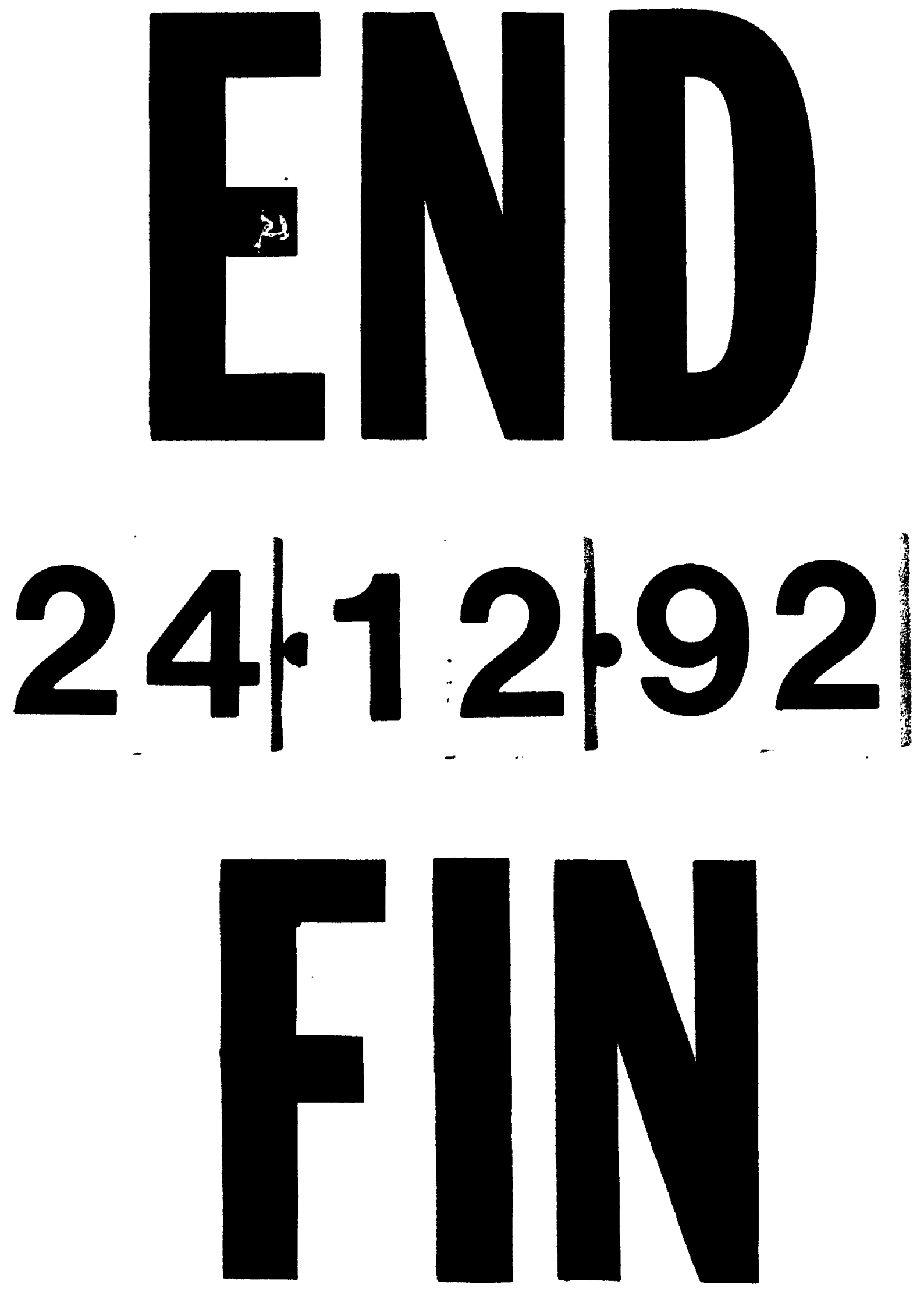